\title{
ULTRASOUND SHEAR WAVE ElASTOGRAPHY: \\ Numerical Modeling And Time-Frequency AnAlysis \\ WITH AN APPLICATION IN HIFU THERMAL LESION DETECTION
}

\author{
by \\ Pooya Sobhe Bidari \\ BSc, Shahid Beheshti University, Tehran, Iran, 2004 \\ A dissertation \\ presented to Ryerson University \\ in partial fulfillment of the \\ requirements for the degree of \\ Doctor of Philosophy \\ in the Program of \\ Electrical \& Computer Engineering
}

MSc, K.N. Toosi University of Technology, Tehran, Iran, 2009

Toronto, Ontario, Canada, 2018

(C) Pooya Sobhe Bidari 2018 


\section{AUTHOR'S DECLARATION FOR ELECTRONIC SUBMISSION OF A DISSERTATION}

I hereby declare that I am the sole author of this dissertation. This is a true copy of the dissertation, including any required final revisions, as accepted by my examiners.

I authorize Ryerson University to lend this dissertation to other institutions or individuals for the purpose of scholarly research.

I further authorize Ryerson University to reproduce this dissertation by photocopying or by other means, in total or in part, at the request of other institutions or individuals for the purpose of scholarly research.

I understand that my dissertation may be made electronically available to the public.

Pooya Sobhe Bidari 


\author{
Ultrasound Shear Wave Elastography: \\ Numerical Modeling and Time Frequency Analysis \\ with an Application in HIFU Thermal Lesion Detection \\ Doctor of Philosophy 2018 \\ Pooya Sobhe Bidari \\ Electrical \& Computer Engineering \\ Ryerson University
}

\begin{abstract}
In this work, a new numerical framework is proposed and implemented to simulate acoustic wave propagation in $3 \mathrm{D}$ viscoelastic heterogeneous media. The framework is based on the elastodynamic wave equation in which a 3D second-order time-domain perfectly matched layer (PML) formulation is developed to model unbounded media. The numerical framework is discretized by a finite difference formulation and its stability analysis is discussed.

The proposed numerical method is capable of simulating 3D shear and longitudinal acoustic waves for arbitrary source geometries and excitations, together with arbitrary initial and boundary conditions. After validation of the framework, it was used to simulate the propagation of ultrasound shear wave in high intensity focused ultrasound (HIFU) induced thermal lesions located within soft tissue. The parameters in these simulations were obtained from standard doubleindentation measurements of the viscoelastic parameters of normal and thermally coagulated chicken breast tissue samples. A HIFU system was used to induce thermal lesions in tissue.

In this study, a new elastography procedure was also introduced to differentiate between the normal and HIFU induced thermal lesions. This method is based on time-frequency analysis of
\end{abstract}


shear wave propagation within the tissue. In the proposed method, the Wigner-Ville distribution has been used as a time-frequency analytical technique to detect the location of shear wave propagating within the tissue, and to estimate the shear speed of the wave as well as its center frequency and attenuation coefficient. This method was applied to the acoustic wave propagation simulation results of the HIFU thermal lesion. It was finally used to estimate the local viscoelastic parameters of the medium. It was demonstrated that the proposed method is capable of differentiating the thermal lesions from the normal tissue based on their viscoelastic parameters. 


\section{Acknowledgements}

First, I would like to thank my supervisors, Dr. Javad Alirezaie and Dr. Jahan Tavakkoli for all the supports during my $\mathrm{PhD}$ studies. I feel privileged to have the opportunity of working with them. I would like to thank the members of my PhD defense committee, Dr. Anthony Sinclair, Dr. Yuan Xu, Dr. Kaamran Raahemifar, Dr. Ling Guan, Dr. April Khademi and Dr. Frank Russo.

I would like to express my sincere appreciation to Dr. Richard Cobbold and Dr. Hisham Assi for all the helpful advice, insightful comments, and guidance throughout my research. I would like to thank Dr. Stephen Waldman and his team for helping me in my experimental studies. I would also like to express my appreciation to Arthur Worthington from Physics department for all the technical supports throughout this work.

I am especially grateful to my parents for always supporting and encouraging me. They were always there for me through all the challenges of life. Last but not least, I would like to express my gratitude to my beautiful wife, Shima, who always encouraged me to pursue my goals and to

continue the hard work. This accomplishment would not have been possible without her unconditional love and supports. 


\section{Table of Contents}

Table of Contents ....................................................................................................... vi

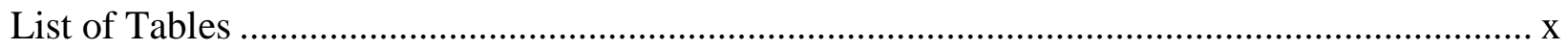

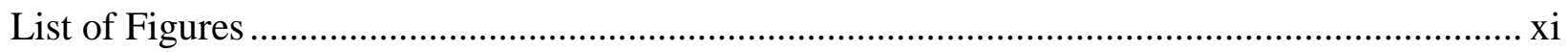

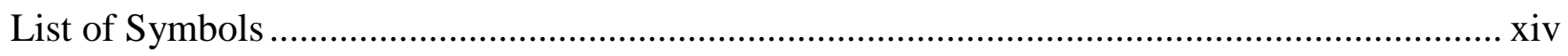

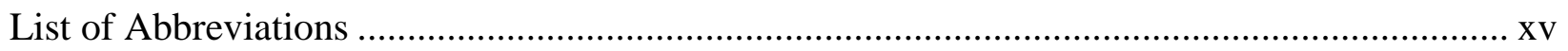

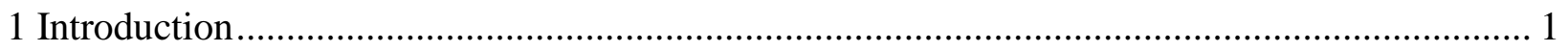

1.1. Motivation ................................................................................................... 1

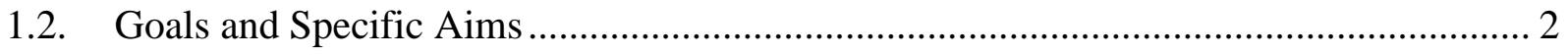

1.3. Main Contributions of the Dissertation .................................................................. 4

1.4. Overview of the Dissertation .............................................................................. 5

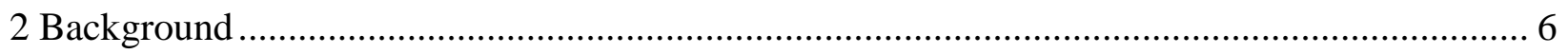

2.1. Biomedical Applications of Ultrasound ............................................................... 6

2.1.1. Diagnostic applications of ultrasound ..................................................... 7

2.1.2. Therapeutic applications of ultrasound .................................................... 10

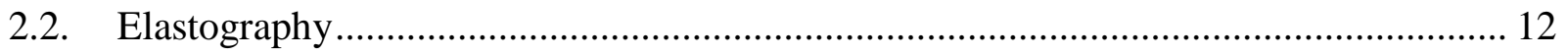


2.2.1. Ultrasound Elastography ............................................................................... 14

2.2.2. Principles of Elastography .............................................................................. 17

2.2.3. Clinical Applications of Elastography ………..................................................... 19

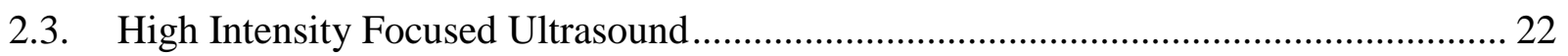

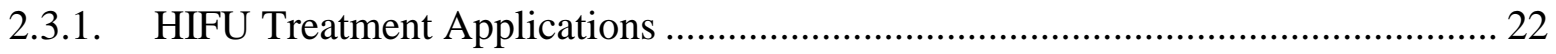

2.3.2. Detecting HIFU thermal lesions ......................................................................... 24

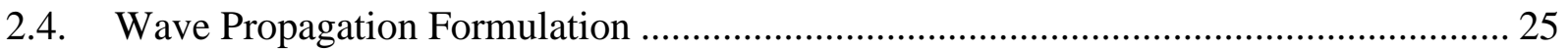

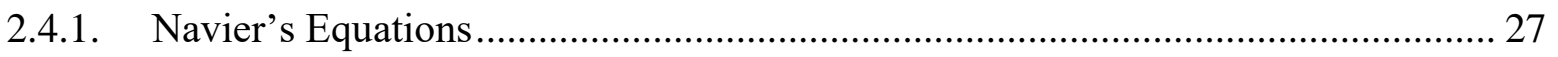

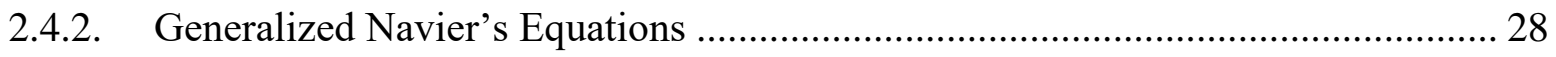

2.4.3. The Elastodynamic Wave Equation .................................................................... 29

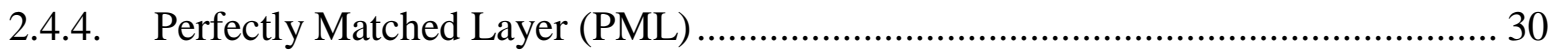

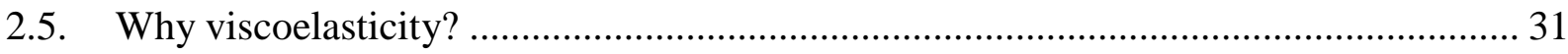

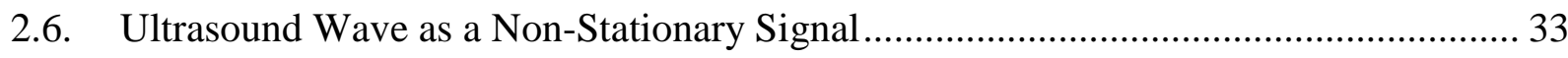

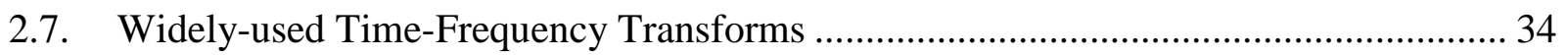

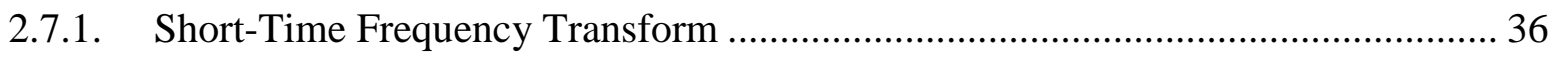

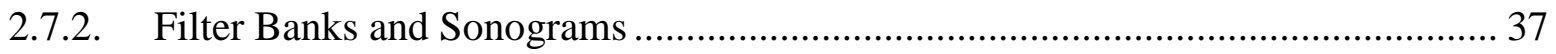

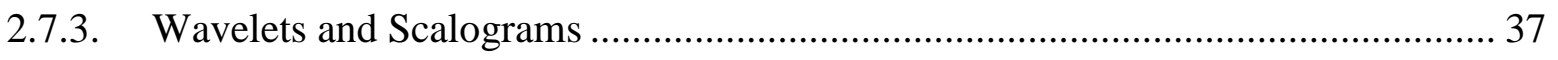

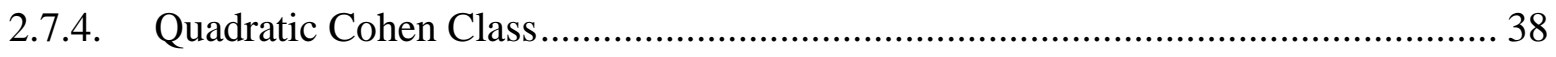

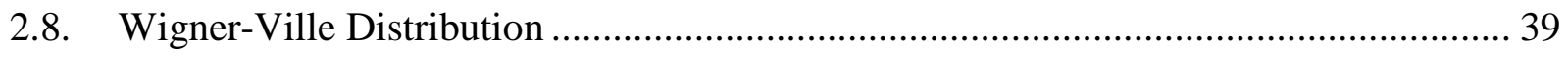

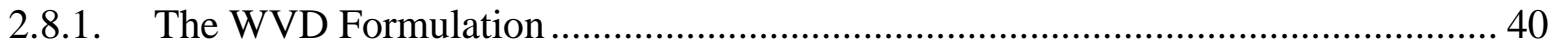


2.8.2. Properties of the WVD

2.8.3. Removing Cross Terms................................................................................... 42

3 Acoustic Wave Propagation Simulation ............................................................................ 44

3.1. Implementation of Viscoelastic Wave Equations ......................................................... 44

3.2. The PML Formulation for the Elastodynamic Wave Equation....................................... 45

3.3. The PML Formulation for the Navier's Equation ......................................................... 49

3.4. Numerical Implementation of the Elastodynamic Wave Equation ................................. 50

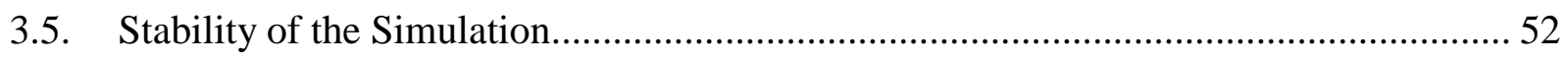

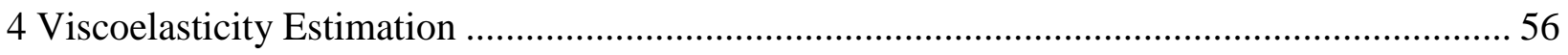

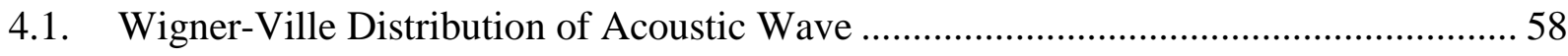

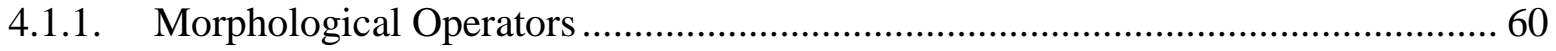

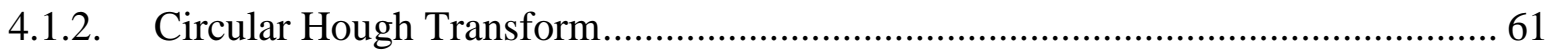

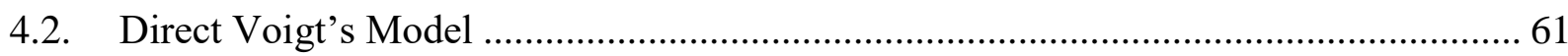

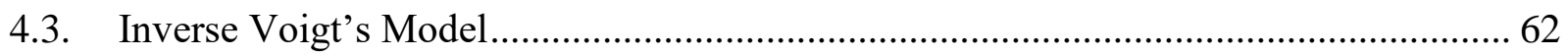

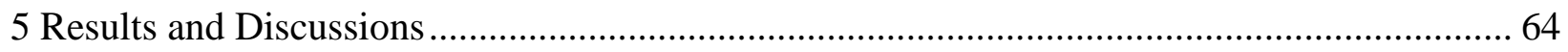

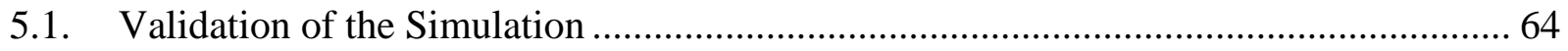

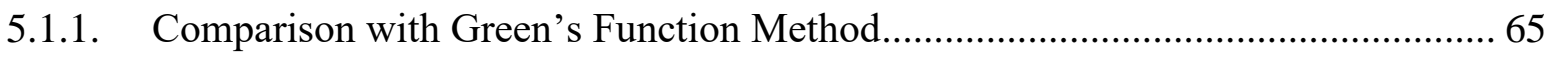

5.1.2. Two-Layer Large Scale Medium Simulation ...................................................... 72

5.2. Simulations of Acoustic Wave Propagation in HIFU Lesion ........................................ 75

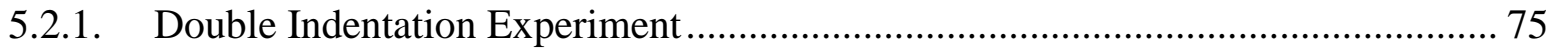


5.2.2. Ultrasound Shear-Wave Simulation in Cubic Inhomogeneous Tissue

5.2.3. Ultrasound Shear-Wave Simulation in Elliptic HIFU Lesion ............................... 82

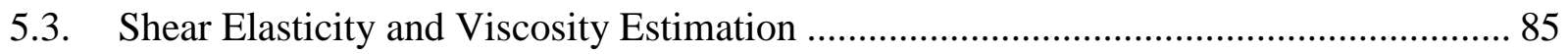

5.3.1. Shear Elasticity and Viscosity Estimation in Elliptical HIFU Lesion ..................... 86

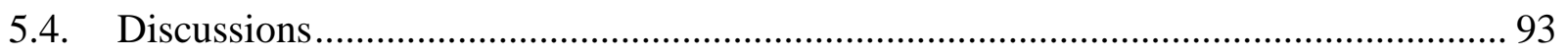

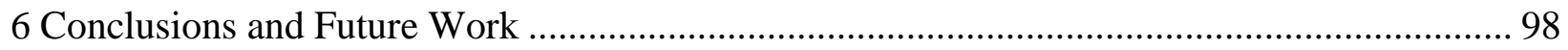

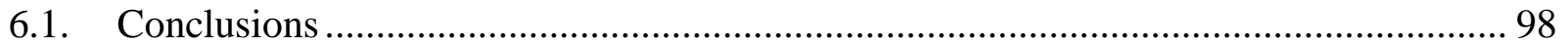

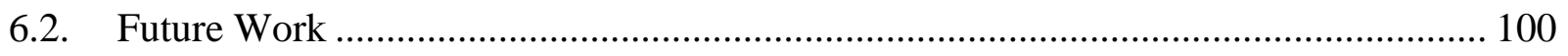

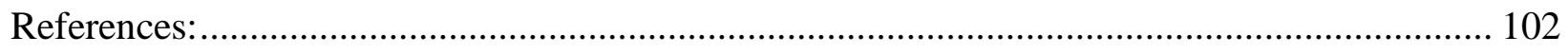




\section{List of Tables}

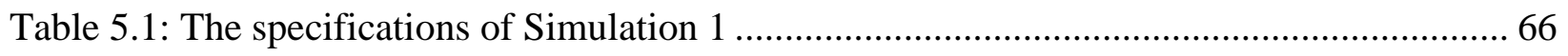

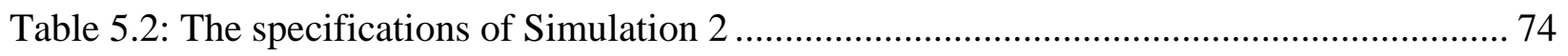

Table 5.3: The results from double indentation experiment ............................................ 78

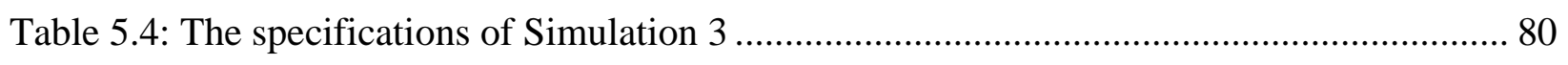

Table 5.5: Parameters used for induced HIFU thermal lesion in chicken breast tissue............... 83

Table 5.6. Acoustic wave parameter captured from time-frequency analysis of 12 sample spatial

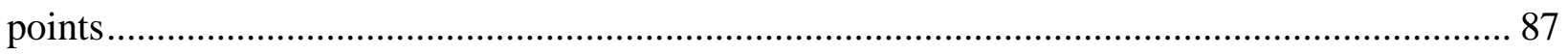




\section{List of Figures}

Figure 2.1: Ranges associated to Shear and Bulk moduli in various materials and body tissue [171].

Figure 5.1: The geometry and the source in the first simulation validation.

Figure 5.2: Comparison between the results of the Green's functions formulation (Bercoff et al. [56]) and the proposed finite difference method using PML at two different radial points. 68

Figure 5.3: Comparison between the results of the Green's functions formulation (Bercoff et al. [56]) and the proposed finite difference method using PML at (a) a lateral point, and (b) an axial point. 69

Figure 5.4: The simulation results with and without PML at two radial points. 70

Figure 5.5: The simulation results with and without PML at (a) a lateral point, and (b) an axial point. 71

Figure 5.6: The comparison of total energy attenuation in time (in decibels) with/without PML.

Figure 5.7: Comparison between results from (a) the proposed method, and (b) the results from Dudouit studies [157] at $\mathrm{t}=50 \mathrm{~ms}, \mathrm{t}=100 \mathrm{~ms}$ and $\mathrm{t}=150 \mathrm{~ms}$. 74

Figure 5.8: (a) HIFU treatment setup, (b) and (c) two pieces of chicken breast with induced HIFU lesions. 77 
Figure 5.9: (a) Samples from HIFU induces lesions and the normal chicken breast tissue, (b) Mach$1^{\mathrm{TM}}$ mechanical tester during double indentation measurement ............................................... 78

Figure 5.10. The simulation geometry: thermal HIFU lesion is inside the normal tissue, and the black area is the PML region 79

Figure 5.11. Shear wave propagation from HIFU thermal lesion to normal tissue regions. The red box denotes boundary of the induced HIFU lesion. 81

Figure 5.12. Shear wave signal at three locations inside the HIFU thermal lesion (Top) and three locations in the normal tissue (Bottom). 81

Figure 5.13. Total energy attenuation in time due to PML 82

Figure 5.14. The simulation geometry: thermal HIFU lesion is inside the normal tissue, and the black area is the PML region 83

Figure 5.15. The simulation geometry: thermal HIFU lesion is inside the normal tissue, and the black area is the PML region. The outline of the elliptical HIFU region has been inserted into each frame. 84

Figure 5.16. Shear wave signal at three locations inside the HIFU thermal lesion (Top) and three locations in the normal tissue (Bottom). 84

Figure 5.17. Total energy attenuation in time due to PML 85

Figure 5.18. Lateral sample points in elliptical HIFU simulation, (top) acoustic signals, (middle) the WVD of the signals, (bottom) captured TF after CHT analysis. 87

Figure 5.19. Axial sample points in elliptical HIFU simulation, (top) acoustic signals, (middle) the WVD of the signals, (bottom) captured TF after CHT analysis. 88

Figure 5.20. Radial sample points in elliptical HIFU simulation, (top) acoustic signals, (middle) the WVD of the signals, (bottom) captured TF after CHT analysis. 89 
Figure 5.21. Time of arrival of the shear wave in elliptical HIFU simulation.

Figure 5.22. (a) speed of shear wave in x direction, (b) speed of shear wave in $\mathrm{z}$ direction, and (c) the total speed of shear wave propagating in the elliptical HIFU simulation......

Figure 5.23. (a) estimated attenuation, (b) estimated shear viscosity, and (c) estimated shear elasticity of the elliptical HIFU simulation........ 


\section{List of Symbols}

\begin{tabular}{ll}
$E$ & Young's Modulus \\
$K$ & Bulk Modulus \\
$\lambda$ & Lame's First Parameter \\
$\mu$ & Lame's Second Parameter \\
$v$ & Poisson Ratio \\
$\mathrm{Pa}$ & Pascal \\
$\eta_{p}$ & Bulk Viscosities \\
$\eta_{s}$ & Shear Viscosities \\
$\vec{u}$ & Displacement Vector \\
$\rho$ & Density \\
$\nabla^{2}$ & Laplacian Operator \\
$\vec{\nabla}$. & Divergence Operator \\
$\nabla$ & Gradient Operator \\
$C_{i j k l}$ & Stiffness Tensor \\
$T F$ & Time-Frequency \\
$\psi(t)$ & Mother Wavelet \\
$\psi_{a, b}(t)$ & Normalized Shifted and Scaled Wavelet \\
$r_{x}(t, \tau)$ & Instantaneous Autocorrelation Function \\
$\tau$ & Delay \\
$\xi$ & Doppler Frequency \\
$W_{z}(t, f)$ & Wigner-Ville Distribution \\
$\mathcal{F}_{\tau \rightarrow f}\{\}$. & Discrete Fourier Transform \\
$z(t)$ & Analytical Associate of the Real Signal \\
$s(t)$ & Real Signal \\
& \\
\hline &
\end{tabular}




\section{List of Abbreviations}

$\begin{array}{ll}\text { ARFI } & \text { Acoustic Radiation Force Impulse } \\ \text { ASF } & \text { Alternating Sequential Filters } \\ \text { CHI } & \text { Contrast-agent Harmonic Imaging } \\ \text { CHT } & \text { Circular Hough Transform } \\ \text { CWD } & \text { Choi-Williams Distribution } \\ \text { CW-Doppler } & \text { Continuous Wave Doppler } \\ \text { DFT } & \text { Discrete Fourier transform } \\ \text { DWT } & \text { Discrete Wavelet Transform } \\ \text { FT } & \text { Fourier Transform } \\ \text { HIFU } & \text { High Intensity Focused Ultrasound } \\ \text { IAF } & \text { Instantaneous Autocorrelation Function } \\ \text { IF } & \text { Instantaneous Frequency } \\ \text { MRE } & \text { Magnetic Response Elastography } \\ \text { MRI } & \text { Magnetic Resonant Imaging } \\ \text { OCT } & \text { Optical Contrast Tomography } \\ \text { PML } & \text { Perfectly Matched Layer } \\ \text { P-Wave } & \text { Primary Wave } \\ \text { PWVD } & \text { Pseudo-WVD } \\ \text { RF } & \text { Radio Frequency } \\ \text { SE } & \text { Structuring Element } \\ \text { SPWVD } & \text { Smoothed-Pseudo-WVD } \\ \text { SSI } & \text { Supersonic Shear Wave Imaging } \\ \text { STFT } & \text { Short-Time Fourier Transform } \\ \text { S-wave } & \text { Secondary Wave } \\ \text { SWE } & \text { Shear-Wave Elastography } \\ \text { TOA } & \text { Time-of-Arrival } \\ \text { TF } & \text { Time-Frequency } \\ \text { THI } & \text { Tissue Harmonic Imaging } \\ \text { WVD } & \text { Wigner-Ville Distribution } \\ & \end{array}$




\section{Chapter 1}

\section{Introduction}

In this chapter, the motivations of this thesis study are first introduced. Then the goals and the specific aims are listed and briefly explained. The contributions of this research are also described and finally an overview of the dissertation is presented.

\subsection{Motivation}

There are various applications of ultrasound in medicine, industry, and science; and they have been rapidly expanding in the past few decades. There is a wide range of non-medical applications of ultrasound such as seismology applications [1], [2], underwater findings, navigation systems, ultrasound welding and flaw detection [3]. Moreover, many research and development works have been carried out in the field of ultrasound in recent years to understand and develop its applications in medicine and biology [4]. There are many biomedical applications of ultrasound ranging from medical diagnosis and imaging techniques [4] to medical therapeutic methods [5].

The simulation of ultrasound wave propagation and its interaction with different types of materials could be a powerful tool for researchers and inventers to develop and improve new ultrasound techniques and methodologies. In this dissertation, an ultrasound wave propagation simulation

method has been proposed based on the elastodynamic wave equation for heterogeneous and 
homogeneous isotropic media. The proposed method is a comprehensive simulation framework which could be very beneficial to avoid time-consuming and expensive ultrasound experiments and the try-and-error approach in the design and implementation of ultrasound applications in different fields.

One of the recent applications of ultrasound in biomedical imaging is elastography. This imaging technique provides information about the local stiffness of soft tissue through analysis of ultrasound wave propagation inside the region of interest and estimation of local elastic or viscoelastic parameters of tissue.

This thesis dissertation is divided into two main parts. In the first part, the focus is to model and numerically simulate ultrasound wave propagation; and in the second part, a new approach in ultrasound elastography is developed to estimate the viscoelastic parameters of soft tissue. The developed method was used toward detection of HIFU thermal lesions in tissue as an application in the field of therapeutic ultrasound.

\subsection{Goals and Specific Aims}

The first goal of this study is to develop an algorithm with the capacities in simulating shear and longitudinal waves propagation in three-dimensional geometries with any arbitrary source geometries and boundary conditions. This method should be capable of simulating elastic and viscoelastic inhomogeneous isotropic media with wide range of medium parameters to cover wide range of applications from biomedicine to seismology. To achieve this goal, a numerical solution of the three-dimensional generalized elastodynamic wave equation has been proposed in this study and has been implemented to simulate full wave propagation in inhomogeneous media. The numerical solution is based on the finite difference technique and has been implemented in a matrix 
form. This method has also been applied to the generalized Navier's wave equation for homogeneous applications. To extend the capacities of the proposed method in simulating boundless media, a perfectly matched layer (PML) formulation has been implemented in the numerical solution to model three-dimensional media with non-reflecting and absorbing boundaries.

The second goal of this study is to utilize the developed simulation tool and model the propagation of ultrasound wave in an induced thermal lesion located within soft tissue. The parameters used in this simulation is based on experimental measurements of high intensity focused ultrasound (HIFU) induced thermal lesions. HIFU is a therapeutic application of ultrasound in which high amount of ultrasonic energy can be concentrated in the focal region of a focused transducer leading to a rapid increase in the local temperature of the focal region until the tissue thermal coagulation occurs.

The third goal of this study is to propose a novel elastography imaging procedure to differentiate between the normal tissue and HIFU induced thermal lesion based on the propagation of shear wave applied inside the thermal lesion. In the proposed method, the Wigner-Ville distribution (WVD) has been used as a time-frequency analytical technique to capture the simulated shear wave propagating in the tissue, to calculate the shear speed of the wave as well as its center frequency and attenuation coefficient, and finally to estimate the local elasticity of the medium. The normal tissue and the thermally coagulated regions could then be differentiated according to difference in their elastic parameters.

The specific aims of this research can be summarized as the following:

1- Development and implementation of a three-dimensional full-wave propagation simulation with the capabilities in modeling isotropic elastic or viscoelastic, homogeneous or 
inhomogeneous, single-layer or multi-layer, bounded or boundless media with any arbitrary acoustic source.

2- Introducing and implementation of PML formulation in the proposed acoustic wave simulation framework.

3- Validation of the proposed simulation and utilizing it to numerically model shear wave propagation within a HIFU induced thermal lesion surrounded by normal tissue.

4- Implementation of an elastography imaging method based on time-frequency analysis of shear wave signals to differentiate the HIFU thermal lesion from the surrounding normal tissue.

\subsection{Main Contributions of the Dissertation}

The major contributions of this work can be summarized as in the following:

1- Developing a numerical simulation framework to model the generalized 3D full-wave acoustic elastodynamic equations including the viscoelastic terms. The model is capable of simulating a wide range of applications such as seismological and biomedical applications.

2- Introducing PML formulations for 3D elastodynamic equations including the viscoelastic term.

3- Simulating shear wave propagation generated from acoustic radiation force in a soft tissue including HIFU thermal lesion with elastography purposes.

4- Introducing a time-frequency analytical method based on the WVD and image processing techniques to locate a shear wave propagating in soft tissue and estimate propagation speed, center frequency of the wave and attenuation coefficient of the medium. 
5- Introducing a HIFU thermal lesion detection method based on time-frequency analysis of acoustic shear wave.

\subsection{Overview of the Dissertation}

In Chapter 2, the ultrasound and the signal analysis backgrounds used in this dissertation are provided. Chapter 2 starts with an overview of the biomedical applications of ultrasound and continues with more detailed descriptions of ultrasound elastography imaging modality and HIFU therapeutic application of ultrasound. The generalized viscoelastic and elastic wave equations as well as the ultrasound background are also presented in Chapter 2. In this chapter, the nonstationary signals and the stablished time-frequency analysis techniques are also reviewed and the Wigner Ville Distribution is explained in more details.

In Chapter 3, the proposed method of full-wave propagation simulation is explained. The implementation of viscoelastic simulation, PML formulation, and stability conditions of the simulation are given in Chapter 3 in details.

In Chapter 4, the time-frequency analysis of the ultrasound wave is studied and the viscoelastic parameters estimation method is described.

The results and discussions of this dissertation are provided in Chapter 5 . In this chapter, the propagation simulation method is validated through quantitative comparison of its results with other published research works. Then, shear wave propagation through a HIFU lesion induced inside soft tissue is simulated. In Chapter 5, the results of WVD time-frequency analysis of the ultrasound wave and the estimation of the medium's local viscoelastic parameters are explained. In Chapter 6, the summary of this study and future works are provided. 


\section{Chapter 2}

\section{Background}

In this chapter, first a brief review of the biomedical applications of ultrasound in diagnosis and treatment is provided. The elastography as an imaging modality and the high intensity focused ultrasound (HIFU) as a treatment application of ultrasound are reviewed in more details. This chapter is continued by reviewing the acoustic wave propagation formulations and their numerical simulation techniques. This provides the background review of the ultrasound researches related to this thesis study.

Then, an explanation is provided to show how the acoustic waves can be treated as non-stationary signals. The well-known time-frequency methods are described and the WVD method is explained in more details. This chapter also provides the basis for the time-frequency analytical method used in this research.

\subsection{Biomedical Applications of Ultrasound}

The propagation of ultrasound wave through the biological tissue involves interaction with the tissue in different ways. The wave may partially be reflected or scattered in the tissue, or be absorbed by the tissue [6]. The reflected and scattered wave echoes from different layers of the tissue can be used as the source of diagnostic information and ultrasound imaging. On the other 
hand, the absorbed wave results in delivery of acoustic energy and can be utilized in therapeutic applications. The biological effect of the absorbed wave is mainly divided into two classes of thermal and non-thermal effects [5]. In the thermal effects, the acoustic energy results in heating the target and rising its temperature [7]. The non-thermal or mechanical effects of ultrasound are cavitation, acoustic radiation force and acoustic streaming [8]. The ultrasound wave can result in formation of micron-sized bubbles in the field, then their activation by the induced pressure wave [7]. At lower intensity levels acoustic streaming is the dominant mechanical mechanism which is a unidirectional high-velocity flow induced by the acoustic pressure [8]. Acoustic radiation force is created from the spatial distribution of the acoustic energy density and it is the result to energy absorption, scattering, and reflection [9].

\subsubsection{Diagnostic applications of ultrasound}

The diagnostic applications of ultrasound have been started to develop since 1940s when the pulseecho ultrasound was used to detect and differentiate tissue structures such as cancer and benign cells, and the gall stones. The first B-mode ultrasound images were generated by Howry and Bliss and Wild and Reid, independently [10]. The diagnostic applications of ultrasound can be categorized as A-mode, B-mode, C-mode, M-mode, continuous-wave Doppler (CW-Doppler), pulsed-Doppler, color-Doppler, power-Doppler, elastography, ultrafast imaging, tissue harmonic imaging (THI) and contrast-agent harmonic imaging (CHI) modalities [4], [11]-[14]. Brief descriptions of these modalities are provided in the following.

A-mode: In this mode, the ultrasound signal is generated by a transducer and propagated through the tissue. Then, the axial echoes from different points of the tissue are displayed as the amplitude versus time showing the strength of reflected signals from different distances [4], [13]. 
B-mode: This is a 2D version of A-mode ultrasound signals when an ultrasound image is created from multiple A-mode lines. The B-mode imaging is a $2 \mathrm{D}$ display of the tissue by representing the strength of the echo signals as the brightness of dots (or pixels) in the image. These images scan the plane through the tissue normal to the surface of the transducer [4], [13]. In order to make a Bmode ultrasound image, the scanner must calculate the time it takes for the ultrasound wave to travel from the surface of the transducer to the scatterrer and back. This shows the distance of the scatterrer from the transducer. The strength of the echo depends on the strength of the transmitted signal and the amount of reflection from the scatterer. A brighter point in the image represents a stronger reflection which could be from a tissue boundary or from a denser area. Stronger transmitted signal could result into a larger depth of wave penetration and a deeper ultrasound image. The echo wave is normally acquired by a predefined sampling rate in the scanner.

In B-mode imaging technique, an array of transducer elements is used. Each element is small size transducer that is capable of both transmitting and receiving. The scanner develops a B-mode image by focusing the transmitted signal electronically by multiple elements of the array transducer, then receiving the echoed signal. A complete image is generated when all transducer elements scan the image line by line. This process limits the maximum number of frames that could be acquired within a second.

C-mode: These images represent the received echoes from a 2D plane in a constant depth of the tissue normal to the B-mode image plane. These images are produced by moving an ultrasound transducer in the surface of the target and using a gate in order to select only the signals from the depth of interest [4], [13]. 
M-mode: In this ultrasound imaging technique, the ultrasound pulses of A-mode or B-mode are sent in quick succession to detect the motion of the target; therefore, the reflections from a moving boundary are gathered to determine its velocity or displacement [4], [13].

Doppler mode: In this category of ultrasound imaging, the Doppler effect is used to measure the blood flow or any motion in the body. In CW-Doppler, continuous ultrasound wave is propagated through the target along a line to detect the velocity of different points on the line. The velocity can be calculated by measuring the changes in the frequency of received signal. In pulsed-Doppler, the sampling is done by sending ultrasound pulses in a specified sample volume considering the time required for the pulse to travel toward the target and return. In color-Doppler, the velocity information is placed on top of a B-mode image in a color coded representation, showing the flow direction toward or away from the transducer according to the frequency or phase shifts in the received signal. Furthermore, in power-Doppler, the frequency and phase shift of the received signal along with its amplitude are utilized to generate the velocity information over the B-mode image [4], [13].

Elastography: This technique was originally an ultrasound imaging method of estimating the tissue elasticity by calculating the local displacements of its elements under a given compression [15]. Elastography was then combined with the other imaging modalities such as Magnetic Resonant Imaging (MRI) and Optical Contrast Tomography (OCT) [16]-[18]. In the conventional ultrasound elastography, the target is subjected to a small compression and the RF echo signals are acquired before and after compression. After comparing these signals, the stiffness of different points along the compression can be estimated. In shear-wave elastography (SWE), the ultrasound signal is focused in a point in the target area as the source of shear wave, and the elasticity of the 
target elements is estimated by calculating the shear wave propagation speed (or pattern) in the tissue [19], [20].

Ultrafast Imaging: In this imaging technique, a single plane wave is transmitted to the region of interest by firing all transducer elements at the same time and then receiving the echoed signals from all the elements. This technique increases the number of frames per second into the range of $5000-15000$ fps. The theory of ultrafast imaging was introduced in 1970s but it has been first developed in a clinical scanner in 2008. This method is based on digital parallel beamforming and needs advanced graphical processing units for implementation [21].

Tissue Harmonic Imaging (THI): This imaging technique is an application of nonlinear ultrasound propagation in tissue. In THI, the spatial resolution of imaging is improved using the second or higher harmonic frequencies generated due to nonlinear wave propagation; moreover, better visualization of smaller objects is obtained in this technique [4], [11]-[13].

Contrast-agent Harmonic Imaging (CHI): In CHI method, the imaging is based on the contrastagent harmonics. The harmonics are generated by reflections from the injected micro-bubbles into the target [22]. When an ultrasound wave hits a gas bubble, the volume of the bubble is changed due to the force from the wave. The changes in the volume result in the generation of harmonics reflections. Then the generated harmonics are utilized in the CHI technique to improve the resolution of imaging [4], [22].

\subsubsection{Therapeutic applications of ultrasound}

Therapeutic applications of ultrasound can be categorized as low and high power classes [8]. At low power levels, beneficial and reversible cellular effects may be produced, whereas at high powers instantaneous cell death is resulted [5]. The low power applications include physiotherapy, 
bone healing and fracture repair, sonophoresis, sonoporation and gene therapy. On the other hand, the high power therapeutic ultrasound in medicine includes lithotripsy, histotripsy and high intensity focused ultrasound (HIFU).

Sonophoresis: It is a process in order to increase the absorption of semisolid compounds in the skin by stimulating it with ultrasound waves. Better delivery of drug through the skin is resulted by the low intensity ultrasound waves as they increase the micro vibrations in the skin cells and the penetration of pharmacologically drugs [5], [23].

Sonoporation: This is another application of low power ultrasound in the drug delivery. In this method, the permeability of the cell plasma membrane is increased by employing acoustic cavitation of micro-bubbles. Then the large molecules of the drug are delivered into the cell for various therapeutic purposes [5], [23].

Gene therapy: It is a similar process to the sonoporation, but the goal is to deliver some modified genes to specific regions of a patient body through increasing the permeability of the cell membrane by ultrasound wave [5].

Physiotherapy, bone healing and fracture repair: These are the other therapeutic applications of low power ultrasound. They are mainly resulted from the periodic nature of the sound pressure field and its massaging effect on tissue [8].

Lithotripsy: This is a technique to break down and damage the stones in the kidney or gallbladder by applying high pressure focused short ultrasound pulses to the target stones. The therapeutic potential of these pulses is due to the formation of destructive shock waves for the disintegration of the stone material without damage to the surrounding tissues [24]. The applied shock waves result in generating cavitation bubbles surrounding the target [25]. 
Histotripsy: In this process, a number of short high intensity ultrasound pulses are focused at the region of interest in the tissue, resulting in mechanical fraction of the target tissue. In this technique, micro-bubbles, that are produced by the high intensity short pulses, are the source of mechanical cavitation in the target [26].

High Intensity Focused Ultrasound: In HIFU, the energy of the focused ultrasound wave is converted into the heat energy through the absorption mechanism at the target tissue that results in increasing of its temperature [27]. The target tissue is positioned at the focal region of the ultrasound transducer. Enough delivery of heat causes the target tissue to be damaged rapidly by coagulation necrosis [5], [8], [28]. The HIFU treatment will be described in more details in Section 2.3 .

\subsection{Elastography}

Elastography is a medical imaging modality that maps biomechanical properties of soft tissue from its responses to a mechanical disturbance. There is important information hidden in the mechanical properties of tissue. The information about the stiffness or softness of tissue in comparison with a normal tissue can provide aids to the physicians to diagnose the existence of an abnormality. From ancient times, manual palpation served as a source of information about soft tissues in order to detect different diseases related to the changes in tissue elasticity. For example, the estimation of tissue stiffness provides key information which can benefit in early detection of abnormalities such as cancerous tumors [29].

During the last two decades, many elasticity imaging methods have been proposed and the applications of elastography have been expanding very fast in medical diagnostics and treatment monitoring. Elasticity imaging methods are emerging as commercial applications. The 
conventional elastography images the local stiffness of tissue by calculating the local displacements of its elements under compression. Elastography has been developed to differentiate the abnormal versus normal tissues by estimating the stiffness variations in the soft tissues [20]. The main idea is from the manual palpation that has been widely used by physicians for detection of abnormalities in different organs. Changes in the elasticity of tissue are generally correlated with pathological phenomena [15].

Elastography has originally developed as an application of ultrasound imaging [15] but it is not limited to ultrasound. Elastography techniques have been also developed by other medical imaging modalities such as MRI and OCT [30]. The goal of elasticity measurements is to evaluate the response of the tissue to the forced displacement or disturbance and to estimate unknown biomechanical properties of soft tissue. This method is completely non-invasive and it can guide or even replace biopsies from tissue. The common biomechanical properties of soft tissue are the bulk modulus, Young's modulus, Poisson's ratio, Lame's first and second coefficients, anisotropy, viscosity and poroelasticity [20]. Elastography imaging has been investigated in many organs and for many diseases. It has been shown that malignant lesions are generally stiffer in comparison with the normal tissue; however, they mostly appear normal in the conventional B-mode ultrasound images [15], [31], [32].

In general, elastography can be considered as an inverse problem of estimating the biomechanical properties of soft tissue by measuring the forced disturbance from a known source of mechanical excitation and using an accurate biomechanical model [20]. Considering this definition, the elastography methods can be characterized based on: 1) the excitation/mechanical source that induces the mechanical displacement within the tissue, 2) the data acquisition technique, 3) the mathematical model and acoustic parameters that are used to model the tissue, and 4) the analytical 
detection method for the local acoustic parameters estimation. In this thesis, these factors are summarized as: 1) the disturbance source, 2) the imaging technique, 3) the model, and 4) the estimation method.

\subsubsection{Ultrasound Elastography}

To measure the elasticity properties of tissue, its behavior under deformation should be monitored. The main methods of inducing a distortion are as follows:

- Pushing/deforming the tissue by an external axial and quasi-static or constant compression,

- Vibrating the surface of the tissue or organ with a probe or an external device,

- Using physiological movements created inside body, e.g. pulse or heartbeat.

- Using acoustic radiation force impulse from an ultrasound transducer to remotely generate push or series of pushes inside the tissue.

These deformation sources are the bases of different ultrasound elastography methods. Most elastography techniques find the tissue stiffness based on one of the following principles:

- Stiffer tissue deforms less than the softer tissue when a force is applied,

- Mechanical shear waves propagate faster within stiffer tissue than softer tissue.

In some elastography method, the distortion of the tissue is displayed. In some other methods, the speed of wave propagation is converted into stiffness map and displayed [20], [33].

Conventional ultrasound elastography involves acquiring RF echo signals (A-lines) from a target region, and subjecting the target to a small compression, e.g. $1 \%$ of its length or less, and obtaining other RF signals along the compression axis. The RF echo signal pairs are then analyzed by piecewise cross-correlation methods in order to calculate the local longitudinal strain profile of the tissue along the compression axis. This calculation results in generating a 2D strain image. By 
combining the estimated local strain values and the applied local stress distribution, a quantitative estimation of local elastic modulus values through the target is resulted [34]. Elastogram is an image obtained by displaying the elasticity information in gray scale [31]. In general, elastograms are local elasticity images produced by processing the pre- and post-compression frames obtained from the target.

The strain imaging, as the simple version of elastography, was first examined on foam phantoms and bacon slabs [15], and later on cancer nodules in the breast and muscles in 1993 [31]; the results showed that the elastography is a promising method in the estimation of strain distributions in phantoms and tissues. It is also stated that elastography is a better modality in detecting lesions compared with B-mode imaging method [15], [31], [34]. In 1994, J. Ophir et al. could identify the beef muscle structures by elastography [35]. The strain map, without considering the distribution of stress, is not a complete indicator of actual elasticity distribution in the target. Pontekani et al. in 1994 [36] and 1995 [37] proposed an estimation of stress distribution in an isotropic elastic target with simple composition, location and boundary conditions assuming that the strain and stress are linearly related.

In the following, the currently-used elastography techniques are reviewed.

\subsubsection{Quasi-Static Elastography:}

The quasi-static elastography method is based on small deformation of the tissue by an external axial force. The deformation is normally in form of a constant compression called quasi-static deformation [20]. During the deformation, several ultrasound RF signals are acquired and the response of the tissue to the deformation is recorded. The pre-compression and post-compression signals are compared in order to produce strain profiles of the scanned region. Local displacement of the tissue is calculated by analyzing the movement of the scatterers between consecutive 
ultrasound RF data. The displacement shows the tissue response to the mechanical force and it is relative to the stiffness of the tissue. The strain map is estimated by determining the displacement from the time shifts between corresponding segments [31]. It is assumed that the softer segments of the tissue take more deformation than the stiffer segments. A strain map is usually shown on top of the B-mode ultrasound image of the same region.

\subsubsection{Transient Elastography and Vibro-Acoustography}

In these methods of elastography, the distortion/displacement is in form of sinusoidal vibration which could be from either a mechanical vibration or an acoustic source. In transient elastography, the source of vibration is a low frequency mechanical vibrator, typically lower than $1 \mathrm{kHz}$, which generates shear wave within the tissue. In vibro-acoustography, acoustic radiation forces are applied to the tissue by two confocal transducers with slightly different frequencies in the range of few hundred Hertz. In both cases, the vibration propagates into the tissue with a speed relative to its stiffness. The tissue response to the vibration is then recorded by ultrasound image acquisition of the region of interest. The image acquisition rate should be high enough to capture the vibration. The main advantage of vibro-acoustography imaging compared to traditional ultrasound imaging is its speckle free characteristic. It also has the ability to image specular surfaces from any angle. Vibro-acoustography has been used to image breast [9], [38], prostate [38], [39], thyroid, liver lesions [40], arteries [41], and bone [42].

\subsubsection{Acoustic Radiation Force Impulse and Shear Wave Elastography}

Acoustic radiation force impulse (ARFI) could be used as the source of deformation in tissue. In this technique, a localized mechanical excitation is induced in the region of interest. The ARFI is usually generated by a focused ultrasound transducer and it is the source of both longitudinal and shear waves propagating in the tissue. The shear modulus of the tissue could be calculated from 
speed of shear wave propagation. Shear modulus is a practical parameter to differentiate different types of soft tissue because it has a broader range of variations than bulk modulus for soft tissues. In shear wave elastography, ultrafast ultrasound image acquisition techniques are used to capture shear wave. Then the local shear wave speed is estimated and it is finally converted into local shear modulus. There are many proposed applications of ARFI such as phantom imaging [43], [44], imaging thermally induced lesions [45], [46], abdominal imaging of cancer lesions [47], [48], prostate imaging [49], and imaging of the cardiovascular system including the heart [50] and vessels [51], [52].

\subsubsection{Supersonic Shear Wave Imaging}

Like ARFI technique, the supersonic shear wave imaging (SSI) relies on the acoustic radiation force from the ultrasound transducer to perturb the tissue [19]. In SSI technique, quasi-plane low frequency shear waves are generated within the soft tissue by the same scanning transducer. The quasi-plane shear waves can be created by rapidly changing the focal point of the acoustic radiation force beam at different depths of the soft tissue along a line. In this technique, it is required to use ultrafast ultrasound image acquisition to capture the shear wave. The data acquisition is normally conducted with 5,000 to 20,000 frames per second. This makes it possible to have a real-time measurement of local shear elasticity within the region of interest. Since the amplitude of the induced shear wave is limited to the power of imaging probe, this method is not suitable for scanning the elasticity of deeper parts of the tissue.

\subsubsection{Principles of Elastography}

An acoustic model of wave propagation in soft tissue is the model that predicts the forced mechanical disturbance (or displacement) in the tissue from a given source when the corresponding 
tissue parameters are known. In other words, if the source of the mechanical excitation is given, the mechanical displacement field can be calculated within the tissue either analytically or numerically knowing an accurate model of the soft tissue with enough knowledge of biomechanical properties. Therefore, the problem of elasticity estimation can be considered as an inverse problem of estimating the biomechanical properties in the model by measuring a forced disturbance [20]. Hook's law, axial strain map [32], axial-shear strain equation [53], shear wave speed equation [54], Voigt's model [55] and the Navier's equation [56] are some examples of the models used in elastography techniques in different studies.

The estimation of biomechanical properties of tissues from various elastographic techniques is based on assuming tissue as a linear viscoelastic and incompressible medium. In viscoelastic models, soft tissue is treated as a solid medium without any fluid motion. On the other hand, tissue can be considered as a combination of fluid phase and porous solid phase. This approach in elastography is called poroelastography [57]-[59].

The elasticity of an inhomogeneous media can be reconstructed in a number of different approaches. In general, these approaches are categorized as direct and indirect reconstruction techniques [60]-[62].

In direct reconstruction technique, the distribution of elastic modulus, e. g. Young's modulus, is calculated by knowing the internal displacement vector and strain tensor at any point within the tissue. The main advantage of this technique is its capability to locally estimate the elastic modulus within the medium without knowing of global boundary conditions.

In indirect approaches, the elastic modulus is estimated mainly based on calculation of the speed of shear wave. In these approaches, the displacement vector is monitored at every point within the tissue, and the speed of shear wave is estimated from the time it takes for the wave to propagate in 
the medium. The indirect approaches could be considered as inverse versions of wave equations. In wave equations, the displacement vector is calculated knowing the elastic and mechanical properties of the medium; on the other hand, in indirect elastography approach, the elastic parameter is calculated by estimating the displacement vector and speed of sound. In general, the elasticity models are based on the general linear equations of dynamic equilibrium that describes the motion of a mechanical body. The Elastodynamic and the Navier's wave equations are described in more details in Section 2.4 and in Chapter 3.

\subsubsection{Clinical Applications of Elastography}

Elastography is an imaging modality that maps differences in the biomechanical properties of normal and abnormal tissues [20]. The strain imaging, as the simple version of elastography, was first examined on foam phantoms and bacon slabs [15], and later on cancer nodules in the breast and muscles in 1993 [31]; the results showed that the elastography is a promising method in the estimation of strain distributions in phantoms and tissues. It is also stated that elastography is a better modality in detecting cancer lesions compared to B-mode imaging method [15], [31], [32]. In 1994, J. Ophir et al. could identify the beef muscle structures by elastography [35].

There have been many clinical practices using elastography since 2005. Most of the ultrasound imaging manufacturers also included elastography to their ultrasound scanners. This technique has been used to examine many tissue types such as breast, prostate, liver, pancreas, thyroid, tendon, muscle, fat, heart, skin, cartilage, blood vessels, lymph nodes, and even brain [63].

The clinical applications of elastography can be categorized into two main groups [20]:

1. Detecting, characterizing and diagnosing diseased or abnormal tissue from normal tissue,

2. Guiding non-invasive or minimally invasive therapeutic techniques. 
It has been shown in many researches that most of pathological or physiological changes in soft tissue results in significant changes in the elasticity. Therefore, elasticity is among the best biomechanical parameters in differentiating abnormal tissue structures [63]. Ultrasound elastography is capable of detecting and distinguishing cancerous tumors from benign lesions [63]. A brief overview of most common elastography clinical applications in recent years has been provided as follows.

\subsubsection{Breast Elastography}

Breast elastography provides non-invasive evaluation of the stiffness of breast lesions in addition to the conventional ultrasound sonography and mammography [64]. The main clinical approaches used as breast elastography are 1) strain elastography and 2) shear wave elastography. It has been demonstrated that elastography assists physicians to better detect cancerous lesions in breast. Since the cancerous cells are stiffer, they are normally darker than benign lesions and surrounding normal breast tissue in elastographic images. A standard method of grading lesion by color maps have also been used in Hitachi scanners to display tissue stiffness [65]. There are methods proposed for qualitative evaluation of stiffness by measuring the strain ratios between the cancerous nodules and the neighboring reference tissue [66]. Estimation of shear modulus of nodules from shear wave velocity provides an assessment of the tissue stiffness [67]-[69].

\subsubsection{Liver Elastography:}

Because the liver is located deep in the body and it is under the rib cage, the application of strain elastography is very limited in liver examinations compared with the superficial organs. Therefore, shear wave elastography techniques are the main elastographic methods in the measurement of liver stiffness. Some common liver elastography examinations are the determination of hepatic cirrhosis, fibrosis and focal liver lesions [29], [70]-[75]. Evaluation of liver fibrosis is usually 
examined by biopsy which is both expensive and invasive. Elastography along with analysis of different blood marker levels is a new non-invasive technique in hepatic fibrosis with promising results in the determination of the degree of liver fibrosis [74]-[77]. Magnetic resonance elastography, transient ultrasound elastography, supersonic shear imaging and shear wave vibrometry are among the methods developed for the assessment of the mechanical properties of liver tissues and fibrosis staging [78]-[80]. The advantages of ultrasound elastography methods in comparison with liver biopsy are that they are rapid, easy, non-invasive and painless.

\subsubsection{Prostate Elastography:}

Prostate cancers are stiff lesions and they are normally not visible in standard ultrasound sonographic images [81]. Therefore, elastography is a useful technique in detecting and locating cancerous regions within the prostate. Two main prostate elastography approaches are 1) the quasistatic method, and 2) the transient shear wave technique [15]. There are several published studies on prostate elastography, but it is generally more difficult to obtain high quality elastographic images of the prostate gland compared with the breast [9], [82], [83]. Shear wave elastography systems that use ARFI or external vibration as the source of shear wave are among the most successful approaches for imaging prostate cancers.

\subsubsection{Thyroid Elastography:}

Malignant thyroid nodules tend to be stiffer than benign nodules [84]. Therefore, thyroid elastography can be potentially used to classify a nodule as malignant or benign [85], [86]. Elastographic imaging of the thyroid can be challenging due to the pulsations from the adjacent carotid artery and due to the steeply sloping neck contour. Elastography combined with different ultrasound imaging modalities has been used to improve basic diagnosis of thyroid [87]. Quasistatic elastography, shear wave and ARFI are used to provide precise mechanical information 
about tissue stiffness, to differentiate malignant and benign nodules and to confirm the presence of Graves disease and chronic autoimmune thyroiditis [78], [88]-[90].

Even though thyroid elastography is still not able to distinguish between different types of thyroiditis, it helps to confirm thyroiditis in general.

\subsection{High Intensity Focused Ultrasound}

High-temperature thermal therapeutic techniques have been applied to many anatomical sites such as brain, eyes, bladder, kidney, and the prostate [28]. These techniques are usually minimally- or non-invasive. In comparison to the traditional open surgeries, the minimally- and/or non-invasive therapeutic approaches have the advantages of reducing tissue damages, side effects and recovery time. There are various modalities to deliver the heat energy to the target area.

HIFU therapy is a minimally- or non-invasive therapeutic approach that has been employed to destroy tumors locally by delivering heat energy and increasing their temperature to above $60^{\circ} \mathrm{C}$ [27], [28]. In the HIFU therapy, the high intensity ultrasound beam is focused and delivered to the targeted tissue and the ultrasound acoustic energy is converted into heat energy through tissue absorption mechanism. Consequently, the temperature of tissue is raised locally to the levels that irreversible structural changes are resulted at that location. HIFU has the capability to deliver energy deep and focalized into the body.

\subsubsection{HIFU Treatment Applications}

In the 1940s, Lynn et al. demonstrated the effects of intensive focused ultrasound beams in biological tissue for the first time [91]. Later, in the 1950s, Burov et al. suggested that HIFU can be used in cancer therapy by inducing coagulated necrosis as the main mechanism responsible for 
tumor treatment. In the 1950s and early 1960, Fry et al. developed HIFU to create tissue destruction specifically in brain and employed it as a treatment of Parkinson`s disease. In the 1980s, HIFU was further employed by Coleman et al. in the treatment of some Ophthalmological problems such as in glaucoma and retinal tears [28]. In the 1980s, after the development of more sophisticated imaging modalities to monitor the procedure of HIFU treatment, this technique was emerged as the subject of interest in many medical researches and was employed on more biomedical areas [5]. Currently, HIFU has many applications in cancer therapy including the treatment of fibro adenoma and cancer of the breast [92], [93], benign prostatic hyperplasia and prostate cancer [94][96], uterine fibroids [97], [98], bladder tumors [99], kidney cancer tumor [100], ovaries, pancreas, and liver cancers [101]. Moreover, HIFU has effective applications in bleeding control and hemostasis of injuries in the solid organs like liver and spleen, and major blood vessels [102]. Recently, HIFU has been used in some non-invasive cosmetic applications such as ablating adipose tissue from the abdomen and flank to reduce the circumference of the waist [103], [104]. HIFU has been also used in facial cosmetic surgery for skin lifting and tightening, wrinkle reduction, and eyebrow lifting [105], [106].

The focal intensity of HIFU beam generated by the transducer can be in the range of $1-10 \mathrm{~kW} / \mathrm{cm}^{2}$ [107]. The high intensity beam is focused at the focal region of the transducer with the small size of few millimeters that results in delivering high energy to this region. The highly focused intensive beam induces thermal and non-thermal (mechanical) biological effects [28] at the focal spot without heating or damaging the surroundings. This mechanism results in the rapid cell death in the tight focal region of the transducer without damaging the other parts of the tissue.

In the thermal effect, the acoustic wave energy is converted to heat energy that increases the temperature of the targeted region. On the other hand, in the non-thermal or mechanical effects, 
high amplitude pressure wave causes the acoustic cavitation that is the formation and activity of destructive gas micro-bubbles around the target [7], [108].

\subsubsection{Detecting HIFU thermal lesions}

Due to the existence of different tissue layers between the HIFU transducer and the targeted area in the tissue, there are always some limitations in the exact estimation of HIFU intensities in a real surgery planning. Besides, the tissue movement and cooling effect of blood flow make the uncertainty of treatment planning even more complicated [109]. Moreover, a complete HIFU treatment generally contains repeated HIFU exposures that scan the whole region to be treated. Therefore, it is necessary to monitor the procedure of HIFU surgery during the treatment in order to make it more efficient and safe as a non-invasive treatment modality, and to ensure that the whole region of interest has been treated.

There are several methods proposed to detect thermal lesions induced by HIFU or to monitor HIFU treatment. B-mode ultrasound imaging has been utilized to monitor the hyper-echoic changes in real-time during the treatment [110]. Analysis of backscattered radio frequency signals have been also studied in some researches to monitor the dynamic changes of tissue during HIFU procedure [111]. The statistical analysis of RF signal was used to monitor the dynamic changes [112] of the acousto-mechanical parameters during and after HIFU treatment. Signal processing methods such as Manifold algorithm [113] have been also used to differentiate ablated tissue from non-ablated tissue during HIFU treatment.

MRI-guided focused ultrasound therapy has been developed during recent years to measure tissue temperature non-invasively and to detect the induced thermal lesion in the tissue [92], [93], [110]. 
Stiffness imaging as a conventional elastography method has been studied in several investigations to quantitatively estimate the size, area and volume of thermal lesions [114], [115]. Magnetic response elastography (MRE) is another proposed method to quantitatively study HIFU lesions by estimating the shear modulus of the tissue (as its elasticity) using MRI technique [116]. In another study, the induced thermal lesion was detected by combining elastography and temperature estimation methods [117]. In this study, a unified computational kernel is proposed to calculate the strain by concurrent estimation of both temperature and elasticity from RF echo time displacements. This technique results in detection of the lesion location and dimension during the HIFU treatment. The feasibility of axial-shear strain elastography has also been studied to visualize the boundaries of HIFU induced lesion [118]. This imaging technique is referred to as axial-shear strain elastography because a quasi-static axial compression is applied to the tissue similar to conventional elastography. Then, the dimension and location of the lesion are estimated by detecting the generated shear wave at the boundaries of the lesion [118].

In a recent study, a shear wave elastography technique using a HIFU transducer was used to generate push beams to monitor the treatment and the tissue coagulation induced by the same transducer. The induced lesion was illustrated in the shear wave map and the results shows that HIFU-push, can produce shear waves strong enough for elastography purposes [119]. An algorithm is needed to accurately measure the shear wave velocities. This would be beneficial to detect the boundaries of the induced lesion and control the treatment process.

\subsection{Wave Propagation Formulation}

The mathematical formulation of wave propagation can be described in the form of particle displacement, particle velocity, pressure, and density. Ultrasound wave propagation in different 
types of tissue has been studied for many years and there are several equations that mathematically model wave propagation in the fluid or solid media. These equations have been used in many studies to model and simulate wave propagation in elastic or viscoelastic, homogeneous or inhomogeneous, bounded or unbounded, single layer or multilayer geometries. The elastodynamic and the Navier's equations have been widely used to model acoustic wave propagation in viscoelastic media in the fields such as seismology researches [1], [2], and biomedical shear wave elastography [56]. In viscoelastic models, soft tissue is treated as a solid medium without any fluid motion; therefore, the Elastodynamic and Navier's equations are decent choices to be used for the ultrasound wave propagation simulation for the elastography purposes. These equations are among the well-known and well-established equations that express the response of a medium to an external source and model the propagation of longitudinal and shear waves.

In this research, the proposed simulation tool covers variety of distribution sources and models the acoustic wave propagation within multilayer inhomogeneous media. The simulation includes full waves, i.e. both longitudinal and shear waves, generated from any arbitrary source in the soft tissue. This can be beneficial for other researches to study different wave detection and estimation methods to calculate the elastography properties of soft tissues before being involved in the experimental validations.

In general, the elasticity of a medium is defined as its ability to resist any form of external mechanical distortion and in different context, there have been different moduli used as elasticity. The well-known parameters that describe elasticity are: Young's modulus $(E)$, bulk modulus $(K)$, Lame's first $(\lambda)$ and second $(\mu)$ parameters, and Poisson ratio $(v)$. Any pair of two moduli from this list is enough to describe the properties of homogeneous isotropic linear elastic medium. The 
rest could be extracted from those two. The definition of these parameters are provided in the following [2]:

Young's Modulus $(\boldsymbol{E})$ : It is a measurement of material stiffness. Young's modulus is defined as the ratio of extensional stress to the resulting extensional strain for a cylinder being pulled on both ends. It can be determined by calculating the slope of stress-strain curve during tensile or compression of the sample material.

Bulk Modulus (K): Buk modulus is defined as the ratio of required hydrostatic pressure to the resulting volume change. It is a measure of the incompressibility or resistance of the material to uniform compression.

Lame's parameters $(\lambda, \mu)$ : The Lame's parameters completely describe the linear stress-strain relation within an isotropic solid. The second Lame's parameter, $\mu$, also known as shear modulus, is defined as half of the ratio between the applied shear stress and the resulting shear strain. This parameter is a measure of the resistance of the material to shearing. The first Lame's parameter, $\lambda$, does not have a physical explanation. This constant is defined based on bulk modulus and shear modulus.

Poisson Ratio (v): It is defined as the ratio of the lateral contraction of a cylinder to its longitudinal extension when it is being pulled on its ends. When a material is stretched in one direction, it tends to get thinner in the other two directions and the Poisson ratio is an indication of this tendency.

\subsubsection{Navier's Equations}

The Navier's equations describe wave propagation in the form of particle displacement in an elastic medium. This equation could be extracted from the Newton's second law and Hooke's 
representation of viscosity stress tensor [56] A linearized version of this equation for an infinite isotropic solid has been shown in Eq. (2-1) [2]:

$$
\rho \frac{\partial^{2} u_{i}}{\partial t^{2}}=\frac{\partial}{\partial x_{i}}\left(\lambda \frac{\partial u_{j}}{\partial x_{j}}\right)+\frac{\partial}{\partial x_{j}}\left(\mu\left(\frac{\partial u_{i}}{\partial x_{j}}+\frac{\partial u_{j}}{\partial x_{i}}\right)\right) .
$$

This equation is a set of three equations for a 3D geometry where index $i=1,2,3$. The Lame's first and second parameters are, respectively, shown as $\lambda$ and $\mu$ and their SI units are the Pascal (Pa). Lame's first parameter can be also described by Young's modulus and Poisson's ratio. Lame's second parameter is also known as shear modulus.

In a homogeneous medium, the Lame's parameters are constant and they could be moved outside the derivative operator. Therefore, the Navier's equation for a homogeneous elastic fluid can be written in vector form as in Eq. (2-2) [56]:

$$
\rho \overrightarrow{\ddot{u}}=\vec{f}+(\lambda+\mu) \overrightarrow{\nabla(\vec{\nabla} \cdot \vec{u})}+\mu \overrightarrow{\nabla^{2} \vec{u}}
$$

this equation is again a set of three equations which model the propagation of both longitudinal and shear waves generated from an external force.

In Eq. (2-2), the external force $\vec{f}$ could be in any arbitrary form. It could also be resulted from multiple sources in any direction and with arbitrary volume and temporal distribution.

\subsubsection{Generalized Navier's Equations}

The generalized viscoelastic version of the Navier's equations could be obtained for an infinite homogenous medium using $\bar{\mu}, \bar{\lambda}$ instead of $\mu, \lambda[56]$ :

$$
\left\{\begin{array}{l}
\bar{\mu}=\mu+\eta_{s} \frac{\partial}{\partial t} \\
\bar{\lambda}=\lambda+\eta_{p} \frac{\partial}{\partial t}
\end{array}\right.
$$


where $\eta_{p}$ and $\eta_{s}$ are the bulk and shear viscosities with the SI units of Pa.s. Bulk viscosity is a property of the medium that measure its internal resistance of compression or expansion. Shear viscosity characterizes the resistance of the medium to shearing flows.

\subsubsection{The Elastodynamic Wave Equation}

The elastodynamic wave equation is the result of combining the equation of motion in a linear elastic solid and Hooke's equation. This equation is described as [1], [2]:

$$
\rho \frac{\partial^{2} u_{i}}{\partial t^{2}}=\sum_{j=1}^{3} \frac{\partial}{\partial x_{j}}\left(\sum_{k, l=1}^{3}\left(C_{i j k l} \frac{\partial u_{k}}{\partial x_{l}}\right)\right)
$$

where $C_{i j k l}$ is the stiffness tensor. This equation is a linear formulation which is valid for wave propagation in any heterogeneous anisotropic medium. It can be simplified for a homogenous medium by considering $C_{i j k l}$ coefficients as constants and moving them outside of the derivatives. As shown in Eq. (2-5), the viscoelastic version of Eq. (2-4) can be obtained by introducing $\eta_{i j k l}$ as the viscosity tensor:

$$
\rho \frac{\partial^{2} u_{i}}{\partial t^{2}}=\sum_{j=1}^{3} \frac{\partial}{\partial x_{j}}\left(\sum_{k, l=1}^{3}\left(C_{i j k l} \frac{\partial u_{k}}{\partial x_{l}}+\eta_{i j k l} \frac{\partial^{2} u_{k}}{\partial t \partial x_{l}}\right)\right)+f_{i}
$$

Using Eq. (2-5), an inhomogeneous viscoelastic medium could be simulated. In the calculation domain, the wave propagation at every spatial point is calculated based on the local parameters. The implementation of these equations is explained in Chapter 3. It should be noted that the numerical algorithms in this thesis study are based on the elastodynamic equation in Eq. (2-5). The simplified cases such as homogeneous simulations of Navier's equation or pure elastic simulations are also based on the implementation of Eq. (2-5). 


\subsubsection{Perfectly Matched Layer (PML)}

In numerical simulations of wave propagation, a discretized domain should be defined to numerically solve the equation under study. This results into computational reflections of the wave from the simulation geometry boundaries. These reflections should be removed when simulating the wave propagation in open boundary media. One technique is to add an absorbing layer surrounding and perfectly matching the computational domain.

The perfectly matched layer (PML) technique was first introduced by Berenger in 1994 [120] to model Maxwell equations and simulate electromagnetic wave propagation. Since then, PML has been applied in several computational studies to simulate the propagation of different types of wave such as electromagnetic [121], [122], seismic, elastic and acoustic waves [123]-[125]. This method has been applied to Maxwell's equations [117], [122], Helmholtz equations [126], Euler equations, the elastodynamic equations [127]-[129].

In general, the goal of using PML formulation is to assure the following two conditions:

1- All frequency components of propagating wave transmit to the PML region without any reflection and at any angle,

2- The wave gets significantly absorbed in the PML region before reaching to the outer boundary of the computational domain.

The following steps are usually performed to obtain the PML formulations for a given wave equation [127]:

1- The Fourier transform of the wave equation with respect to time is calculated.

2- New dependent complex variables are introduced to allow absorption in the PML region. 
3- The introduced variables are set to zero in the physical domain and non-zero in the PML.

4- The resulting formulations are simplified and split into main and auxiliary equations to model the wave propagation in both physical and PML regions.

5- The equations are transferred to time-domain by inverse Fourier transform.

The resulted formulations will include main equations similar to the original wave equation including PML variables, and some auxiliary equations that present wave propagation in the PML region. All the resulting equations should be numerically solved together with the Finite Difference or the Finite Element methods to include the PML region in the simulation. Since the PML variables are zero in the physical domain, the wave propagation simulation results are correct. The PML equations are solved only at the boundaries and they do not affect the actual physical layer.

\subsection{Why viscoelasticity?}

As previously mentioned, HIFU is a technique that utilizes intensive focused ultrasonic waves to elevate the temperature at the focal region in a very short period of time. This procedure as a noninvasive surgical technique must be used alongside with a reliable monitoring technique to localize the induced thermal lesion to accurately verify the extent of tissue damage. The detection of HIFU thermal lesions during the treatment provides a feedback to determine whether the desired location is being sufficiently ablated and to make appropriate changes in the treatment plan, if needed. This is a challenging problem to be solved because it is difficult to precisely measure the volume and boundaries of the thermal lesion in real time during the HIFU treatment. It is even more complex 


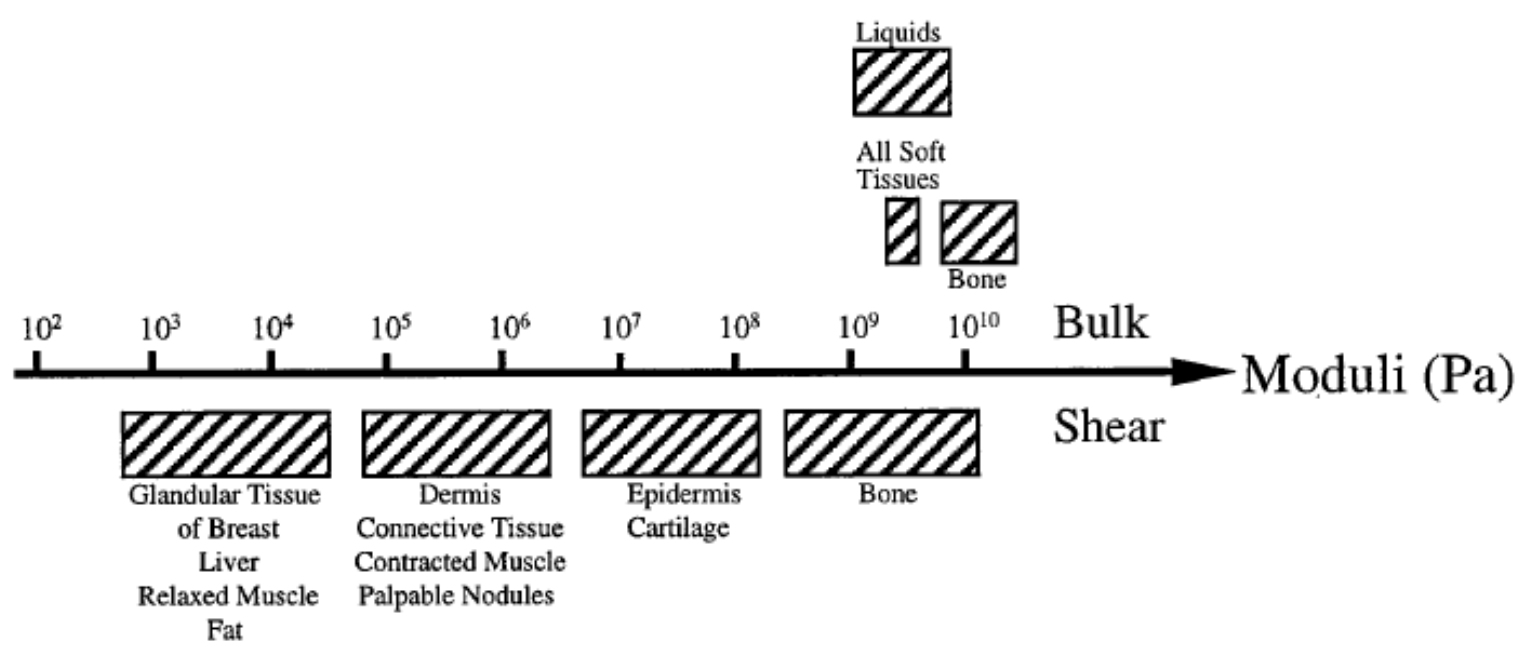

Figure 2.1: Ranges associated to Shear and Bulk moduli in various materials and body tissue [171].

because the response of the tissue to increased temperature is not immediate and the boundaries between the coagulated region and the normal tissue is not well defined.

The propagation of shear wave within the tissue can provide quantified information about the stiffness of the normal tissue or the lesions inside it. The goal of shear wave elastography, as a method in characterization of soft tissue elasticity, is to generate shear stiffness images from the soft tissue to detect the abnormal tissue [20], [54], [130]-[132]. This method is based on measurement of shear wave propagation speed within the area of interest. The shear wave speed is 2 - 4 times larger in abnormal diseased tissue than its speed in the normal tissue [54]. In shear wave elastography, it is an advantage to use the wide range of shear moduli in comparison with bulk moduli in the soft tissue. This wide range has been shown in Figure 2.1.

The HIFU thermal lesions are stiffer than normal surrounding soft tissue and the estimation of shear modulus leads us to differentiate the thermal lesion from its surrounding tissue [133]. In this research, the wide range of shear modulus has been used as an advantage to differentiate a simulated HIFU thermal lesion from the surrounding normal tissue.

A new method is proposed in this study based on the time-frequency analysis of the shear wave to measure the viscoelastic parameters in the region of interest to improve HIFU thermal lesion 
detection. In the proposed method, the viscoelastic parameters of the tissue are estimated at every spatial point independent to the other points. The WVD time-frequency method used in this thesis can capture the shear wave propagating in the tissue which results in calculating the shear speed of the wave as well as its frequency and attenuation coefficient. This technique provides more insight and quantitative information about the medium elastic properties; thus, the normal tissue and the thermally coagulated regions could be differentiated according to difference in their elastic parameters.

\subsection{Ultrasound Wave as a Non-Stationary Signal}

As the complexity of the information carried in a signal grows, it becomes more essential to select a representation space in which the information could be more clearly observed, structured, and processed. In fact, the choice of a representation space is an important decision in processing the signals. The perception of time-frequency signal analysis methods is to analyze and process signals with time-varying frequency content [134], [135].

The acoustic wave generated by radiation force impulse is a non-stationary signal with timevarying frequencies because it normally contains both longitudinal and shear components with different speeds and frequencies. These wave components travel in time and they might be present at a specific location just in a short period [136], [137]. Moreover, because the biological tissue shows dispersive properties in regards to acoustic wave propagation, the tissue displacement and the shear wave generated from an acoustic radiation force are frequency dependant [138]. All these properties emphasize that the ultrasound wave shows non-stationary behaviour in the shear wave elastography applications [139]. 
Such signals are better represented by a time-frequency distribution which illustrate the distribution of the energy of the signal over the two-dimensional time and frequency domain rather than only time or frequency domains. The time-frequency distribution illustrates the frequency range of the signal and the variation in frequency within time. This variation is called the instantaneous frequency (IF). To better understand the behaviour of ultrasound elastographic wave propagation, there is need to utilize a robust time-frequency analytical solution.

The focus of this chapter is to provide more detailed information about the time-frequency analysis signals generated by acoustic radiation force impulses in the shear wave elastography.

\subsection{Widely-used Time-Frequency Transforms}

The representation of a signal in time domain does not show the frequency components of the signal. On the other hand, The Fourier transform represents only the spectral contents of the signals without any demonstration of localization in time. Time and frequency domains have both limitations when dealing with non-stationarity signals such as acoustic signals. In order to overcome these limitations and to analyze the non-stationary signals, there is need for techniques that provide joint time and frequency information. The time-frequency (TF) representation indicates a two-dimensional energy demonstration of a signal in terms of time and frequency. In these methods, the signal is decomposed into elementary functions that are well concentrated in time and frequency domains. These elementary functions localize the signal not just by a pure frequency, but by packets of close frequencies in time.

There are many joint-TF techniques proposed in different studies. The suitability of each technique depends on the nature of the signal and its complexity [140]-[143].

In general, the desirable characteristics of a time-frequency domain technique are as follows [144]: 
- Capability to identify the characteristics of the signal such as time variation, frequency variation, number of components, relative amplitudes, etc.

- Capability to separate the components from each other and from the noise.

- Capability to analyze specific components separately, and to track the instantaneous amplitude, the instantaneous frequency, and the instantaneous bandwidth.

- Capability to employ a mathematical model that shows the significant characteristics of the signal, such as the IFs.

In linear time-frequency transforms, the correlation of the signal by a family of waveforms is calculated. The concentration of the waveforms in time and frequency indicate the strength of the method in localizing the TF components. These waveforms are called time-frequency atoms [145]. It is necessary to have different types of atoms to be able to analyze different signal structures. In the following, a brief description of popular time-frequency transforms is explained.

The most widely-used time-frequency analysis techniques could be classified as:

- Short-time Fourier transform or spectrograms [146], [147];

- Filter banks or sonograms [147];

- Wavelets: Continuous such as Morlet wavelet, or discrete such as Haar wavelet [145];

- Quadratic Cohen class: Wigner-Ville distribution (WVD), Choi-Williams distribution (CWD), Page distribution (as an instantaneous power spectrum method), Rihaczek and Levin distributions (as energy density methods) [134], [141];

- Dictionary learning methods [148].

Any of these techniques transform a temporal signal into a TF space where the TF values show the strength of the located frequency component at the given time stamp. Depending on the 
transformation technique, different properties of the signal could be obtained from the TF distributions.

For example, some methods construct distributions with non-negative entries while some might result in negative values. Some methods have better energy localization in TF space and some results in generation of cross-terms [141].

\subsubsection{Short-Time Frequency Transform}

The Short-time Fourier transform (STFT) is a windowed version of the Fourier transform where a moving window is multiplied to the signal in the Fourier transform integral calculations. This results in concentration of the Fourier transform into specific time locations. In general, the STFT of signal $f(t)$ can be defined as follows [145]:

$$
S f(u, \xi)=\int_{-\infty}^{+\infty} f(t) g(t-u) e^{-j \xi t} d t,
$$

in this equation, the signal $f(t)$ is multiplied to $g(t-u)$ which is zero everywhere except for the neighborhood of $t=u$. This results in the localization of the Fourier Transform in different time instances by changing the value of $u$. The function $g(t)$ is called the windowed Fourier atom and $g(t-u)$ is the shifted version in time.

The energy density of STFT is called spectrogram and it indicates the energy distribution of the signal in time and frequency plane. A very special case of STFT is Gabor transform where the window function is in form of a Gaussian function:

$$
G f(u, \xi)=\int_{-\infty}^{+\infty} f(t) e^{-(t-u)^{2} /\left(2 \sigma^{2}\right)} e^{-j \xi t} d t .
$$

Dennis Gabor introduced this transform in 1946 which was the basis of the STFT [146]. 


\subsubsection{Filter Banks and Sonograms}

A filter bank is a set of band-pass filters that are applied to the input signal in order to decompose the signal into the sub-signal components with sub-frequency details. Sonograms are the results of applying filter banks to the signal and they could be considered as the window-based presentation of the signals in frequency domain [141]. In order to generate the sonogram representation of a signal and extract its bandpass component, the Fourier transform of the signal is multiplied to the centered filter functions in frequency domain; then, the inverse Fourier is calculated. Although this process is nonlinear, it does not result into artifacts.

\subsubsection{Wavelets and Scalograms}

A wavelet is a wave-like function that has a concentrated energy in time. Wavelets are used to analyze non-stationary, time-varying and transient phenomena [145]. Wavelets can be defined in different forms depending on the application. They are used to transform a signal into a new representation that present useful information about the signal. When a signal is convolved with a specific wavelet, the result shows the time points that the signal has the most similarity to the wavelet. A set of complementary wavelets is normally needed to analyze a signal. This concept gives us a powerful mathematical tool to deal with non-stationary signals.

In wavelet analysis of the signals, a mother wavelet is normally defined with a specific function; then, the wavelet family is constituted by shifting and scaling the mother wavelet. Depending on the definition of the mother wavelet, there are different methods proposed by researchers in this field. When a signal is visually represented by Wavelets, the visual display of energy density is called scalogram [145]. 
If the scaling and shifting procedures are performed in a continuous fashion, the result will be Continuous Wavelet Transforms (CWT); on the other hand, in Discrete Wavelet Transforms (DWT) the scaling and shifting are executed in discrete steps.

The CWT of a continuous signal $f(t)$ is defined as [149]:

$$
T(a, b)=\int_{-\infty}^{+\infty} f(t) \psi_{a, b}^{*}(t) d t=\left\langle f, \psi_{a, b}\right\rangle
$$

where $\psi(t)$ is the mother wavelet function and $\psi_{a, b}(t)=\frac{1}{\sqrt{a}} \psi\left(\frac{t-b}{a}\right)$ is the normalized shifted and scaled wavelet extracted from the mother wavelet.

The most widely-used continuous wavelet transforms are Morlet, Mexican hat, Meyer, Poisson and Shannon wavelets [145], [149]. The difference between these transforms is the definition of mother wavelet function.

The DWT of a signal $f(t)$ is defined as [149]:

$$
\begin{gathered}
T_{m, n}=\int_{-\infty}^{+\infty} f(t) \psi_{m, n}(t) d t=\left\langle f, \psi_{m, n}\right\rangle, \\
\psi_{m, n}(t)=\frac{1}{\sqrt{a_{0}^{m}}} \psi\left(\frac{t-n b_{0} a_{0}^{m}}{a_{0}^{m}}\right),
\end{gathered}
$$

where $a_{0}$ is specified fixed step parameter, and the integer values $m$ and $n$ control the discretization of the wavelet. The widely-used discrete wavelet transforms are Haar, Daubechies and Dual-tree Complex Wavelet transforms [145].

\subsubsection{Quadratic Cohen Class}

In general, the quadratic Cohen class of a signal is a set of every bilinear representation covariant under time and frequency translations and are formulated in the following form:

$$
C_{x}(t, f)=\int_{-\infty}^{+\infty} \int_{-\infty}^{+\infty} r_{x}(t, \tau) e^{-j 2 \pi f \tau} d v d t
$$

where, 


$$
r_{x}(t, \tau)=\phi_{t-d}(t-v, \tau) x\left(v+\frac{\tau}{2}\right) x^{*}\left(v-\frac{\tau}{2}\right) .
$$

In fact the Cohen class is the Fourier transform of an instantaneous autocorrelation function defined as $r_{x}(t, \tau)$. In this equation $\phi_{t-d}$ represent different types of kernel in the Cohen quadratic class. The equation can be written in the following form after defining narrow band symmetric ambiguity function $A_{x}(\tau, \xi)$ and the use of Fourier transform:

$$
\begin{gathered}
C_{x}(t, f)=\int_{-\infty}^{+\infty} \int_{-\infty}^{+\infty} \phi_{t-D}(\tau, \xi) A_{x}(\tau, \xi) e^{j 2 \pi(t \xi-f \tau)} d \xi d t, \\
A_{x}(\tau, \xi)=\int_{-\infty}^{+\infty} x\left(t+\frac{\tau}{2}\right) x^{*}\left(t-\frac{\tau}{2}\right) e^{-j 2 \pi t \xi} d t, \\
\phi_{t-D}(\tau, \xi)=\int_{-\infty}^{+\infty} \phi_{t-d}(\tau, \xi) e^{-j 2 \pi t \xi} d t .
\end{gathered}
$$

In the above equations, $\tau$ and $\xi$ represent the shift in time and frequency and they are known as the delay and Doppler frequency, respectively. The ambiguity function is a function of the input signal and does not depend on the method. On the other hand, different definition for the kernel results in different types of time-frequency methods [147]. The widely-used time-frequency methods in the Cohen class are: the Wigner-Ville distribution, Rihaczek distribution, Levin distribution, Page distribution and Choi-Williams distribution [141].

\subsection{Wigner-Ville Distribution}

The Wigner-Ville distribution is a time-frequency analytical method with the best time and frequency resolution for a non-stationary process [141], [150]. This method is under the Cohen's class of TF analysis methods and has a quadratic nature. The WVD is based on a symmetrical instantaneous autocorrelation function (IAF) that extracts the localized and Instantaneous Frequency content of a non-stationary signal [151]. Since the acoustic wave propagation is a non- 
stationary signal with time varying frequency content, the WVD seems to be a suitable approach to detect the shear waves [139].

The WVD, as a time-frequency analysis method, has been studied to detect the low-frequency shear waves that were modulated by the acoustic radiation force of ultrasound resulted from two transducers with slightly different frequencies [139]. The WVD has been utilized to analyze Doppler spectrum both in biomedical and radar applications [152], [153] and seismic wave parameter estimation [154] in previous studies. This analysis method has also been studied in researches in estimation of the motion parameters of objects either in the air or in the water based on the acoustical waves generated from the objects. It has been shown in [155] that a TFD with optimal energy concentration is capable to estimate the frequency and the speed of the moving object under water by time-frequency analysis of acoustic data.

The main drawback associated to this method is the production of cross-terms (interferences) when there are more than one time-frequency components in the signal. The cross-terms are the artifacts in between the existing time-frequency components, which are formed due to the quadratic nature of the WVD [151]. The cross-terms are exactly in the middle of any pair of existing time-frequency components. To reduce the interference and the cross-terms, the WVD utilizes the analytical associate of the real signal.

\subsubsection{The WVD Formulation}

The WVD of a signal $s(t)$ is shown by $W_{z}(t, f)$ and is defined in Eq. (2-16) [141]:

$$
W_{z}(t, f)=2 \mathcal{F}_{\tau \rightarrow f}\left\{z\left(t+\frac{\tau}{2}\right) z^{*}\left(t-\frac{\tau}{2}\right)\right\},
$$

where $z(t)$ is the analytical associate of the real signal $s(\mathrm{t})$ at a given local point. $z(t+\tau / 2)$ and $z(t-$ $\tau / 2)$ are the shifted versions of the signal to the left and right sides in time domain, and $\mathcal{F}_{\tau \rightarrow f}\{$.$\} is$ 
the discrete Fourier transform with respect to the shift. The WVD of a signal contains the IFs at any time window. The analytical associate of a real signal is its complex-valued version where the negative frequency components are removed.

\subsubsection{Properties of the WVD}

The WVD has several desirable attributes in signal processing applications. Some of the most important properties of the WVD are listed in the following:

- Generality: Since the WVD has the simplest form of time-lag kernel, other TF distributions can be extracted from it by applying specific time-lag filters.

- Realness: The WVD is real for all values of $t, f$ and $z$.

- Time-shift invariance: A time shift in the signal results in the same amount of time shift in the WVD.

- Time delay: The periodic first moment of the WVD with respect to time is called the time delay.

- Frequency-shift invariance: A frequency shift in the signal results in the same amount of frequency shift in the WVD.

- Instantaneous frequency: The periodic first moment of the WVD with respect to frequency is the instantaneous frequency.

- Time marginal: The integration (summation) of the WVD over frequency results in the instantaneous power of the signal at even time samples [150]:

$$
\int_{-\infty}^{+\infty} W_{z}(t, f) d f=|z(t)|^{2} .
$$

- Spectral marginal: The integration (summation) of the WVD over time results in the energy spectrum of the signal at even frequency samples [150]: 


$$
\int_{-\infty}^{+\infty} W_{Z}(t, f) d t=|Z(f)|^{2} .
$$

- Time support: If $z(t)=0$ everywhere except $t_{1}<t<t_{2}$, then $W_{z}(t, f)=0$ everywhere except $t_{1}<t<t_{2}[141]$.

- Frequency support: If $Z[k]=0$ everywhere except $f_{1}<f<f_{2}$, then $W_{z}(t, f)=0$ everywhere except $f_{1}<f<f_{2}[141]$.

Even with all desirable properties of WVD, it has some drawbacks. Since the WVD is a bilinear or quadratic operation, it suffers from the cross-term artifacts which appears when dealing with signals with multi TF components. The cross-terms appear in the midway between the true TF components. They make the WVD difficult to interpret if there are several TF components in the signal or if they are close to each other. The cross-terms also make it more difficult to interpret the WVD in the presence of noise.

\subsubsection{Removing Cross Terms}

The cross terms can be partially removed or supressed by introducing window-based WVD. The proposed methods to eliminate or reduce the cross-terms are listed in the following:

1- Masked WVD [141]: This is the result of multiplying the WVD by the Spectrogram. The WVD has high TF resolution but includes cross-terms; on the other hand, the spectrogram has lower resolution but it does not have any cross-term. In masked WVD, the high resolution of the WVD is combined with the cross-term suppression of the spectrogram. This results into cleaner time-frequency distribution than the WVD.

2- Multiplying by the Fourier transform of the signal. This results into suppression of the cross-terms by filtering them in frequency domain [141], [147]. 
3- Pseudo-WVD [134], [141], [147]: In this method, a sliding window is applied in the time domain before calculation of the WVD. This results in suppression of the cross-term by averaging a WVD in time.

4- Smoothed-pseudo-WVD [134], [141], [147]: In this method, a sliding averaging window function is applied in the time-frequency plane. This results in more smoothing of the crossterm component. Smoothed-pseudo-WVD method deemphasizes the components arising from calculations and gives more emphasis to the deterministic components.

In the pseudo-WVD and smoothed-pseudo-WVD, the averaging window could be defined in various forms. The most frequently used options are Gaussian, Hamming or Hanning windows. There are two main drawbacks in pseudo- and smoothed-pseudo- WVD in comparison with the original version. First, the computational cost of applying a moving window is high and it reduces the speed of calculation. Second, using an averaging window reduces the timefrequency resolution and localization. The wider the window introduces more averaging; therefore, the cross-terms are reduced more but they are less localized. This means that applying averaging window decreases the time and frequency resolution. 


\section{Chapter 3}

\section{Acoustic Wave Propagation Simulation}

In this chapter, the numerical implementations of the elastodynamic wave equation and its homogeneous version, the Navier's equation, are described. The numerical solutions are based on the finite difference method to model the ultrasound wave propagation in 3D heterogeneous isotropic media with any arbitrary shape acoustic source. The procedure of obtaining the perfectly matched layer (PML) formulation for the simulation is also presented.

\subsection{Implementation of Viscoelastic Wave Equations}

The three equations representing acoustic wave propagation in an infinite homogenous viscoelastic medium known as Navier's equations are shown in Eq. (3-1) [56]:

$$
\begin{aligned}
& \rho \frac{\partial^{2} u_{1}}{\partial t^{2}}=(\bar{\mu}+\bar{\lambda})\left(\frac{\partial^{2} u_{1}}{\partial x_{1}^{2}}+\frac{\partial^{2} u_{2}}{\partial x_{1} \partial x_{2}}+\frac{\partial^{2} u_{3}}{\partial x_{1} \partial x_{3}}\right)+\bar{\mu}\left(\frac{\partial^{2} u_{1}}{\partial x_{1}^{2}}+\frac{\partial^{2} u_{1}}{\partial x_{2}^{2}}+\frac{\partial^{2} u_{1}}{\partial x_{3}^{2}}\right)+f_{1}, \\
& \rho \frac{\partial^{2} u_{2}}{\partial t^{2}}=(\bar{\mu}+\bar{\lambda})\left(\frac{\partial^{2} u_{1}}{\partial x_{1} \partial x_{2}}+\frac{\partial^{2} u_{2}}{\partial x_{2}^{2}}+\frac{\partial^{2} u_{3}}{\partial x_{2} \partial x_{3}}\right)+\bar{\mu}\left(\frac{\partial^{2} u_{2}}{\partial x_{1}^{2}}+\frac{\partial^{2} u_{2}}{\partial x_{2}^{2}}+\frac{\partial^{2} u_{2}}{x_{3}^{2}}\right)+f_{2}, \\
& \rho \frac{\partial^{2} u_{3}}{\partial t^{2}}=(\bar{\mu}+\bar{\lambda})\left(\frac{\partial^{2} u_{1}}{\partial x_{1} \partial x_{3}}+\frac{\partial^{2} u_{2}}{\partial x_{2} \partial x_{3}}+\frac{\partial^{2} u_{3}}{\partial x_{3}^{2}}\right)+\bar{\mu}\left(\frac{\partial^{2} u_{3}}{\partial x_{1}^{2}}+\frac{\partial^{2} u_{3}}{\partial x_{2}^{2}}+\frac{\partial^{2} u_{3}}{\partial x_{3}^{2}}\right)+f_{3} .
\end{aligned}
$$

The first and second terms in the right side of these equations represent the longitudinal and shear waves, respectively. These waves are also known as P-wave and S-wave in seismology contexts 
[1]. As mentioned in previous chapters, the generalized Navier's equations could be obtained for an infinite homogenous viscoelastic medium using $\bar{\mu}, \bar{\lambda}$ instead of $\mu, \lambda$ from Eq. (2-3).

\subsection{The PML Formulation for the Elastodynamic Wave Equation}

In the simulating of ultrasound wave propagation with open boundaries, there could be unwanted numerical reflections from the boundaries of the computational domain. In order to model wave propagation in unbounded infinite media, these computational reflections should be eliminated. One of the most effective solutions to this problem is the perfectly matched layer (PML) formulation [120], [128], [156]-[158]. This method along with finite difference or finite element numerical simulations could model ultrasound wave propagation in unbounded infinite media. The idea is to add an artificial absorbing layer to the computational domain that is perfectly matched to the boundaries of the physical domain. This results in the dissipation of the propagating wave in the PML region with negligible reflections at the boundaries. It requires adding some additional auxiliary PML formulation to the wave equation in order to model the absorbing layer. The PML equations are solved only at the boundaries and they do not affect the actual physical layer.

In this study, a similar approach to the method proposed by Assi et al. [128], [156] is used to obtain the PML formulations for the elastodynamic wave equation and to its homogeneous case, the Navier's equation. Assi et al. proposed PML formulations for 2D elastic wave equation in their research study. In this work, a generalized PML formulation has been proposed for 3D viscoelastic medium.

The following steps summarize the method to obtain the PML formulations:

1- Mapping the elastodynamic wave equation into the complex domain: 


$$
\rho \frac{\partial^{2} u_{i}}{\partial t^{2}}=\sum_{j=1}^{3} \frac{\partial}{\partial \tilde{x}_{j}}\left(\sum_{k, l=1}^{3}\left(C_{i j k l} \frac{\partial u_{k}}{\partial \tilde{x}_{l}}+\eta_{i j k l} \frac{\partial^{2} u_{k}}{\partial t \partial \tilde{x}_{l}}\right)\right)
$$

In this equation $\tilde{x}$ is the complex transformation of the coordinates $x$, where $\tilde{x}_{j}$ depends only on $x_{j}$

2- Fourier Transform of the elastodynamic equation including the PML terms: In this step, the Fourier transform of Eq. (3-2) is calculated:

$$
\rho\left((-i \omega)^{2} U_{i}\right)=\sum_{j=1}^{3} \frac{\partial}{\partial \tilde{x}_{j}}\left(\sum_{k, l=1}^{3}\left(C_{i j k l} \frac{\partial U_{k}}{\partial \tilde{x}_{l}}+(-i \omega) \eta_{i j k l} \frac{\partial U_{k}}{\partial \tilde{x}_{l}}\right)\right)
$$

3- Introducing complex function $s_{j}\left(x_{j}\right)$ to map the derivatives between the complex and the real coordinates. Assuming a dependent variable $U$ :

$$
\begin{gathered}
\frac{\partial U_{i}}{\partial x_{j}}=\frac{\partial U_{i}}{\partial \tilde{x}_{j}} \frac{\partial \tilde{x}_{j}}{\partial x_{j}}=s_{j}\left(x_{j}\right) \frac{\partial U_{i}}{\partial \tilde{x}_{j}}, \\
\frac{\partial}{\partial \tilde{x}_{j}}=\frac{1}{s_{j}\left(x_{j}\right)} \frac{\partial}{\partial x_{j}}
\end{gathered}
$$

where the complex stretch function, $s_{j}\left(x_{j}\right)$, can be defined as:

$$
s_{j}\left(x_{j}\right)=\alpha_{j}\left(x_{j}\right)\left[1+i \frac{\beta_{j}\left(x_{j}\right)}{\omega}\right]
$$

In Eq. (3-6), $\alpha_{j}$ is called the scaling parameter and $\beta_{j}$ is the damping coefficient. In this work, no scaling is considered, but only damping in the defined PML region is defined. Thus; $\alpha_{j}=1, \beta_{j}=$ 0 in the physical domain and $\alpha_{j}=1, \beta_{j} \geq 0$ in the absorbing layer. Hence, the complex coordinate transform can be simply achieved by applying the following equation to any spatial derivative in the wave equation:

$$
\frac{\partial}{\partial \tilde{x}_{j}}=\frac{1}{\left[1+\frac{\beta_{j}\left(x_{j}\right)}{-i \omega}\right]} \frac{\partial}{\partial x_{j}}
$$

one of the commonly used choices for $\beta_{j}$ is: 


$$
\begin{gathered}
\beta_{j}\left(x_{j}\right)=\left\{\begin{array}{cc}
0 & \left|x_{j}\right| \leq x_{0} \\
\beta_{0 j}\left(\frac{\left|x_{j}\right|-x_{0}}{d}\right)^{n} & x_{0}<\left|x_{j}\right| \leq x_{0}+d
\end{array},\right. \\
\frac{\partial}{\partial \tilde{x}_{j}}=\frac{1}{\left[1+\frac{\beta_{j}\left(x_{j}\right)}{-i \omega}\right]} \frac{\partial}{\partial x_{j}},
\end{gathered}
$$

where $\beta_{0 j}$ is a constant. For a normal incident $\beta_{0 j}$ can be expressed in terms of the desired maximum reflection coefficient $\left(R_{j}\right)[128]$ :

$$
\beta_{0 j}\left(x_{j}\right)=\frac{c_{\max }(n+1)}{2 d} \ln \left(1 / R_{j}\right) .
$$

In this equation $c_{\max }$ is the maximum speed of wave propagation in the medium and $n$ is the order of the equation for $\beta_{j}$. The speed of propagation of longitudinal and shear waves can be estimated by the following equations:

$$
\begin{gathered}
c_{L}=\sqrt{\frac{\lambda+2 \mu}{\rho}}, \\
c_{S}=\sqrt{\frac{\mu}{\rho}} .
\end{gathered}
$$

Using the following equations, the final formulation of the perfectly matched layer will be obtained:

$$
\begin{aligned}
& s_{1}\left(x_{j}\right) s_{2}\left(x_{j}\right) s_{3}\left(x_{j}\right)=\left[1+\frac{\beta_{1}}{-i \omega}\right]\left[1+\frac{\beta_{2}}{-i \omega}\right]\left[1+\frac{\beta_{3}}{-i \omega}\right]=\frac{1}{(-i \omega)^{3}}\left[(-i \omega)^{3}+\right. \\
& \left.(-i \omega)^{2}\left(\beta_{1}+\beta_{2}+\beta_{3}\right)+(-i \omega)\left(\beta_{1} \beta_{2}+\beta_{1} \beta_{3}+\beta_{2} \beta_{3}\right)+\beta_{1} \beta_{2} \beta_{3}\right], \\
& s_{j}\left(x_{j}\right) s_{l}\left(x_{j}\right)=\left[1+\frac{\beta_{j}}{-i \omega}\right]\left[1+\frac{\beta_{l}}{-i \omega}\right]=\frac{1}{(-i \omega)^{2}}\left[(-i \omega)^{2}+(-i \omega)\left(\beta_{j}+\beta_{l}\right)+\beta_{j} \beta_{l}\right], \\
& \frac{s_{1} s_{2} s_{3}}{s_{l}}=\frac{1}{(-i \omega)^{2}}\left[(-i \omega)^{2}+(-i \omega)\left(\beta_{1}+\beta_{2}+\beta_{3}-\beta_{l}\right)+\frac{\beta_{1} \beta_{2} \beta_{3}}{\beta_{l}}\right],
\end{aligned}
$$




$$
\begin{aligned}
& \frac{s_{1} s_{2} s_{3}}{s_{l} s_{j}}=\frac{\left[1+\frac{\left(\beta_{1}+\beta_{2}+\beta_{3}-\beta_{l}\right)}{(-i \omega)}+\frac{\beta_{1} \beta_{2} \beta_{3}}{(-i \omega)^{2} \beta_{l}}\right]}{\left[1+\frac{\beta_{j}}{-i \omega}\right]}=\frac{\left[(-i \omega)+\left(\beta_{1}+\beta_{2}+\beta_{3}-\beta_{l}\right)+\frac{\beta_{1} \beta_{2} \beta_{3}}{(-i \omega) \beta_{l}}\right]}{\left[-i \omega+\beta_{j}\right]}=1+ \\
& \frac{\left[\left(\beta_{1}+\beta_{2}+\beta_{3}-\beta_{l}-\beta_{j}\right)+\frac{\beta_{1} \beta_{2} \beta_{3}}{(-i \omega) \beta_{l}}\right]}{\left[-i \omega+\beta_{j}\right]} .
\end{aligned}
$$

4- Substituting $\tilde{x}$ with $x$ using Eq. (3-3) and multiplying the resulted equation by $s_{1} s_{2} s_{3}$ :

$$
\rho s_{1} s_{2} s_{3}\left((-i \omega)^{2} U_{i}\right)=\sum_{j=1}^{3} \frac{\partial}{\partial x_{j}}\left(\sum_{k, l=1}^{3} \frac{s_{1} s_{2} s_{3}}{s_{j} s_{l}}\left(C_{i j k l} \frac{\partial U_{k}}{\partial x_{l}}+(-i \omega) \eta_{i j k l} \frac{\partial U_{k}}{\partial x_{l}}\right)\right) .
$$

5- Simplifying the left side of Eq. (3-17) as:

$$
\begin{aligned}
& \rho s_{1} S_{2} S_{3}\left((-i \omega)^{2} U_{i}\right)=\rho\left[(-i \omega)^{2}+(-i \omega)\left(\beta_{1}+\beta_{2}+\beta_{3}\right)+\left(\beta_{1} \beta_{2}+\beta_{1} \beta_{3}+\right.\right. \\
& \left.\left.\beta_{2} \beta_{3}\right)+\frac{\beta_{1} \beta_{2} \beta_{3}}{(-i \omega)}\right] U_{i},
\end{aligned}
$$

and, calculating the inverse Fourier transform of this formulation as:

$$
\rho\left[\frac{\partial^{2} u_{i}}{\partial t^{2}}+a \frac{\partial u_{i}}{\partial t}+b u_{i}+c \int u_{i} d \tau\right]
$$

where:

$$
a=\left(\beta_{1}+\beta_{2}+\beta_{3}\right), \quad b=\left(\beta_{1} \beta_{2}+\beta_{1} \beta_{3}+\beta_{2} \beta_{3}\right), \quad c=\beta_{1} \beta_{2} \beta_{3} .
$$

6- Simplifying the right side of Eq. (3-17) as:

$$
\begin{aligned}
& \sum_{j=1}^{3} \frac{\partial}{\partial x_{j}}\left(\sum_{k, l=1}^{3} \frac{s_{j} s_{l}}{s_{1} s_{2} s_{3}}\left(C_{i j k l} \frac{\partial U_{k}}{\partial x_{l}}+(-i \omega) \eta_{i j k l} \frac{\partial U_{k}}{\partial x_{l}}\right)\right)=\sum_{j=1}^{3} \frac{\partial}{\partial x_{j}}\left(\sum_{k, l=1}^{3}[(1+\right. \\
& \left.\frac{\left(\beta_{1}+\beta_{2}+\beta_{3}-\beta_{l}-\beta_{j}\right)+\frac{\beta_{1} \beta_{2} \beta_{3}}{(-i \omega) \beta_{l}}}{-i \omega+\beta_{j}}\right)\left(C_{i j k l} \frac{\partial U_{k}}{\partial x_{l}}+\right. \\
& \left.\left.\left.(-i \omega) \eta_{i j k l} \frac{\partial U_{k}}{\partial x_{l}}\right)\right]\right)=\sum_{j=1}^{3} \frac{\partial}{\partial x_{j}}\left(\sum_{k, l=1}^{3}\left(C_{i j k l} \frac{\partial U_{k}}{\partial x_{l}}+(-i \omega) \eta_{i j k l} \frac{\partial U_{k}}{\partial x_{l}}\right)+W_{i j}\right),
\end{aligned}
$$

and, calculating the inverse Fourier transform of this side of the equation as: 


$$
\sum_{j=1}^{3} \frac{\partial}{\partial x_{j}}\left(\sum_{k, l=1}^{3}\left(C_{i j k l} \frac{\partial u_{k}}{\partial x_{l}}+\eta_{i j k l} \frac{\partial^{2} u_{k}}{\partial t \partial x_{l}}\right)+w_{i j}\right)
$$

where $W_{i j}$ is defined by the following equation in the Fourier domain:

$$
W_{i j}=\sum_{k, l=1}^{3}\left[\left(\frac{\left(\beta_{1}+\beta_{2}+\beta_{3}-\beta_{l}-\beta_{j}\right)+\frac{\beta_{1} \beta_{2} \beta_{3}}{(-i \omega) \beta_{l}}}{-i \omega+\beta_{j}}\right)\left(C_{i j k l} \frac{\partial U_{k}}{\partial x_{l}}+(-i \omega) \eta_{i j k l} \frac{\partial U_{k}}{\partial x_{l}}\right)\right] .
$$

By taking the inverse Fourier transform, the equation for $w_{i j}$ in time domain can be obtained:

$$
\begin{aligned}
& \frac{\partial w_{i j}}{\partial t}+\beta_{j} w_{i j}=\sum_{k, l=1}^{3}\left[\left(\left(a-\beta_{l}-\beta_{j}\right) C_{i j k l} \frac{\partial u_{k}}{\partial x_{l}}+\frac{c}{\beta_{l}} \eta_{i j k l} \frac{\partial u_{k}}{\partial x_{l}}+\right.\right. \\
& \left.\left.\left(a-\beta_{l}-\beta_{j}\right) \eta_{i j k l} \frac{\partial^{2} u_{k}}{\partial t \partial x_{l}}+\frac{c}{\beta_{l}} C_{i j k l} \frac{\partial \int u_{k} d \tau}{\partial x_{l}}\right)\right] .
\end{aligned}
$$

Therefore, the 3D viscoelastic wave equation that includes PML will be in the following formulation:

$$
\begin{aligned}
& \rho\left[\frac{\partial^{2} u_{i}}{\partial t^{2}}+a \frac{\partial u_{i}}{\partial t}+b u_{i}+c \int u_{i} d \tau\right]=\sum_{j=1}^{3} \frac{\partial}{\partial x_{j}}\left(\sum_{k, l=1}^{3}\left(C_{i j k l} \frac{\partial u_{k}}{\partial x_{l}}+\eta_{i j k l} \frac{\partial^{2} u_{k}}{\partial t \partial x_{l}}\right)+w_{i j}\right) \\
& \frac{\partial w_{i j}}{\partial t}+\beta_{j} w_{i j}=\sum_{k, l=1}^{3}\left[\left(\left(a-\beta_{l}-\beta_{j}\right) C_{i j k l} \frac{\partial u_{k}}{\partial x_{l}}+\frac{c}{\beta_{l}} \eta_{i j k l} \frac{\partial u_{k}}{\partial x_{l}}+\left(a-\beta_{l}-\beta_{j}\right) \eta_{i j k l} \frac{\partial^{2} u_{k}}{\partial t \partial x_{l}}+\right.\right. \\
& \left.\left.\frac{c}{\beta_{l}} C_{i j k l} \frac{\partial \int u_{k} d \tau}{\partial x_{l}}\right)\right]
\end{aligned}
$$

This formulation includes three main equations for $u_{i}$ and nine auxiliary equations for $w_{i j}$, where $i, j=1,2,3$.

\subsection{The PML Formulation for the Navier's Equation}

The Navier's equation could be extracted from the elastodynamic wave equation. By comparing equations (2-1) and (2-4), the similarity between the coefficients can be obtained. This is summarized as follows: 


$$
\begin{aligned}
& C_{i i i i}=\lambda+2 \mu, \quad C_{i i j j}=\lambda, \quad C_{i j i j}=C_{i j j i}=\mu, \\
& \eta_{i i i i}=\eta_{p}+2 \eta_{s}, \quad \eta_{i i j j}=\eta_{p}, \quad \eta_{i j i j}=\eta_{i j j i}=\eta_{s},
\end{aligned}
$$

where $i, j=1,2,3, \quad i \neq j$. All other $C_{i j k l}$ and $\eta_{i j k l}$ coefficients are zero. Using the equation set Eq. (3-25), and substituting the coefficients in Eq. (3-26), the PML formulation can be obtained for the Navier's equations. It should be noted that the coefficients in Eq. (3-25) are all local values.

\subsection{Numerical Implementation of the Elastodynamic Wave Equation}

In this section, the numerical implementation of the elastodynamic wave equation including the PML formulation, Eq. (3-25), is explained. This implementation is valid for 3D inhomogeneous isotropic media and a homogeneous counterpart can be simply extracted. It should be noted that, since Eq. (3-25) is valid for anisotropic materials, the proposed method can also be used for anisotropic media. However, the focus of this study is on isotropic materials.

1- Parameter initialization:

Every parameter related to the wave formulation or mechanical properties of simulation domain are defined as a 3D matrix to make the simulation capable of dealing with heterogeneous conditions. This includes defining the following 3D matrices:

- $\quad \rho(x, y, z), \lambda(x, y, z), \mu(x, y, z), \eta_{p}(x, y, z)$ and $\eta_{s}(x, y, z)$.

- $\quad u_{x}, u_{y}$ and $u_{z}$ as the three components of displacement vector at every local point, their first and second derivatives in time and space, as well as their integral in time.

2- Calculating $c_{\max }, \beta_{01}, \beta_{02}$ and $\beta_{03}$ :

In equations (3-8) to (3-10), the PML length, $d$, is usually a value between $10 \%$ to $20 \%$ of the length of the physical layer. $\beta_{1}, \beta_{2}$ and $\beta_{3}$ are then initialized as 3D matrices. 
3- Determining the stability of the simulation based on the proposed method in the next section.

4- Defining the source parameters:

In this simulation framework, one or multiple sources can be defined. Each source can be defined by a 3D matrix in space to specify its location, geometry, and shape, and a $1 \mathrm{D}$ matrix in time that specifies the excitation signal.

5- Iterative wave propagation calculation:

This step is mainly based on Eq. (3-25) and the calculation of wave propagation is performed iteratively in time. The new displacement values at time $t+1$ for each local point depend on the previous displacement values at $t$ and the neighboring points.

In Eq. (3-25), the first derivatives in space are first calculated based on the backward explicit difference; then, after multiplication to the mechanical coefficient the second derivatives are calculated based on forward difference formulations.

In each iteration, $w_{i j}$ coefficients are first calculated as 3D matrices, then, the displacement components are calculated based on these coefficients, the displacement spatial and temporal derivatives, and displacement integral in time. All these matrices are then updated and saved for the next iteration.

The above 5-step process results in the implementation of elastodynamic wave equation in 3D. Since it is an iterative process, the amount of memory used for the calculations only depends on the spatial resolution of the simulation. On the other hand, the calculation time depends on both the temporal and the spatial resolutions.

As mentioned in Chapter 2, Navier's equation is the homogeneous case of the elastodynamic wave equation. Therefore, the proposed numerical method is also valid for homogeneous simulations. 


\subsection{Stability of the Simulation}

Considering the numerical stability of the simulation, it is required to select the time and spatial increments $(\Delta t, \Delta h)$ carefully to satisfy the convergence conditions of the finite difference method. The spatial gridding, $\Delta h$, should be small enough to capture the shortest wavelength existing in the simulation; moreover, the time step, $\Delta t$, should be small enough to avoid numerical instability of the simulation in the iterating calculations. Therefore, $\Delta h$ depends on the frequency content of the source, and it can be chosen based on the shortest wavelength:

$$
\Delta h \leq 0.1 \lambda_{\min } .
$$

The proper choice of $\Delta t$ can be obtained by writing the finite difference equivalent of Eq. (2-2). Since the viscosity terms act as damping parameters in this equation, $\Delta t$ can be calculated only based on the elastic part of equation. In the other words, the numeric specifications resulting in stability of the elastic simulation should make the viscoelastic wave simulation stable too. The finite difference elastic version of Eq. (2-2) in $z$ direction is shown in Eq. (3-28):

$$
\begin{aligned}
\rho \frac{u_{z}(x, y, z, t+\Delta t)-2 u_{z}(x, y, z, t)+u_{z}(x, y, z, t-\Delta t)}{(\Delta t)^{2}} \\
=\mu \nabla^{2} u_{z}(x, y, z, t)+(\mu+\lambda) \nabla_{z}(\vec{\nabla} \cdot \overrightarrow{\mathrm{u}}(x, y, z, t)) .
\end{aligned}
$$

This equation could be written in form of Eq. (3-29) after converting the spatial second derivative operators into their equivalent discrete forms, using the Von Neumann stability analysis approach. In Von Neumann approach, the discrete terms are substitute by their Fourier series at a discrete point $(x, y, z)[159]$ :

$$
\begin{aligned}
& \left(u_{z, x y z}^{t+1}-2 u_{z, x y z}^{t}+u_{z, x y z}^{t-1}\right)= \\
& \frac{(\Delta t)^{2}}{\rho} \frac{\mu\left(u_{z, x y z}^{t}\right)\left(e^{i k \Delta h}+e^{-i k \Delta h}+e^{i l \Delta h}+e^{-i l \Delta h}+e^{i m \Delta h}+e^{-i m \Delta h}-6\right)}{(\Delta h)^{2}}+
\end{aligned}
$$




$$
\begin{aligned}
& \frac{(\Delta t)^{2}}{\rho} \frac{(\lambda+\mu)\left(u_{x, x y z}^{t}\right)\left(e^{i(k+m) \Delta h}+e^{-i(k+m) \Delta h}-e^{i(k-m) \Delta h}-e^{-i(k-m) \Delta h}\right)}{4(\Delta h)^{2}}+ \\
& \frac{(\Delta t)^{2}}{\rho} \frac{(\lambda+\mu)\left(u_{y, x y z}^{t}\right)\left(e^{i(l+m) \Delta h}+e^{-i(l+m) \Delta h}-e^{i(l-m) \Delta h}-e^{-i(l-m) \Delta h}\right)}{4(\Delta h)^{2}}+ \\
& \frac{(\Delta t)^{2}}{\rho} \frac{(\lambda+\mu)\left(u_{z, x y z}^{t}\right)\left(e^{i m \Delta h}+e^{-i m \Delta h}-2\right)}{(\Delta h)^{2}}=A_{z x} u_{x, x y z}^{t}+A_{z y} u_{y, x y z}^{t}+A_{z z} u_{z, x y z}^{t},
\end{aligned}
$$

where $\vec{u}_{x y z}^{t}=\left[u_{x, x y z}, u_{y, x y z}, u_{z, x y z}\right]^{T}=\left[e^{i(k x)}, e^{i(l y)}, e^{i(m z)}\right]^{T}$ denotes the particle displacement vector at point $(x, y, z)$ at time $t$ when $x=n_{x} \Delta h, y=n_{y} \Delta h$, and $z=n_{z} \Delta h$.

The same approach can be used in $x$ and $y$ directions:

$$
\begin{aligned}
& \left(u_{x, x y z}^{t+1}-2 u_{x, x y z}^{t}+u_{x, x y z}^{t-1}\right)= \\
& \frac{(\Delta t)^{2}}{\rho} \frac{\mu\left(u_{x, x y z}^{t}\right)\left(e^{i k \Delta h}+e^{-i k \Delta h}+e^{i l \Delta h}+e^{-i l \Delta h}+e^{i m \Delta h}+e^{-i m \Delta h}-6\right)}{(\Delta h)^{2}}+ \\
& \frac{(\Delta t)^{2}}{\rho} \frac{(\lambda+\mu)\left(u_{y, x y z}^{t}\right)\left(e^{i(l+k) \Delta h}+e^{-i(l+k) \Delta h}-e^{i(l-k) \Delta h}-e^{-i(l-k) \Delta h}\right)}{4(\Delta h)^{2}}+ \\
& \frac{(\Delta t)^{2}}{\rho} \frac{(\lambda+\mu)\left(u_{z, x y z}^{t}\right)\left(e^{i(m+k) \Delta h}+e^{-i(m+k) \Delta h}-e^{i(m-k) \Delta h}-e^{-i(m-k) \Delta h}\right)}{4(\Delta h)^{2}}+ \\
& \frac{(\Delta t)^{2}}{\rho} \frac{(\lambda+\mu)\left(u_{x, x y z}^{t}\right)\left(e^{i k \Delta h}+e^{-i k \Delta h}-2\right)}{(\Delta h)^{2}}=A_{x x} u_{x, x y z}^{t}+A_{x y} u_{y, x y z}^{t}+A_{x z} u_{z, x y z}^{t}, \\
& \left(u_{y, x y z}^{t+1}-2 u_{y, x y z}^{t}+u_{y, x y z}^{t-1}\right)= \\
& \frac{(\Delta t)^{2}}{\rho} \frac{\mu\left(u_{y, x y z}^{t}\right)\left(e^{i k \Delta h}+e^{-i k \Delta h}+e^{i l \Delta h}+e^{-i l \Delta h}+e^{i m \Delta h}+e^{-i m \Delta h}-6\right)}{(\Delta h)^{2}}+ \\
& \frac{(\Delta t)^{2}}{\rho} \frac{(\lambda+\mu)\left(u_{x, x y z}^{t}\right)\left(e^{i(k+l) \Delta h}+e^{-i(k+l) \Delta h}-e^{i(k-l) \Delta h}-e^{-i(k-l) \Delta h}\right)}{4(\Delta h)^{2}}+ \\
& \frac{(\Delta t)^{2}}{\rho} \frac{(\lambda+\mu)\left(u_{z, x y z}^{t}\right)\left(e^{i(m+l) \Delta h}+e^{-i(m+l) \Delta h}-e^{i(m-l) \Delta h}-e^{-i(m-l) \Delta h}\right)}{4(\Delta h)^{2}}+ \\
& \frac{(\Delta t)^{2}}{\rho} \frac{(\lambda+\mu)\left(u_{y, x y z}^{t}\right)\left(e^{i l \Delta h}+e^{-i l \Delta h}-2\right)}{(\Delta h)^{2}}=A_{y x} u_{x, x y z}^{t}+A_{y y} u_{y, x y z}^{t}+A_{y z} u_{z, x y z}^{t} .
\end{aligned}
$$


Combining Eqs. (3-29) to (3-31) lead us to the following matrix based equation:

$$
\vec{u}_{x y z}^{t+1}-2 \vec{u}_{x y z}^{t}+\vec{u}_{x y z}^{t-1}=A \vec{u}_{x y z}^{t}
$$

where $A_{3 \times 3}=\left[\begin{array}{ccc}A_{x x} & A_{x y} & A_{x z} \\ A_{y x} & A_{y y} & A_{y z} \\ A_{z x} & A_{z y} & A_{z z}\end{array}\right]$ :

$$
\begin{aligned}
& A_{x x}=\frac{(\Delta t)^{2}}{\rho} \frac{\mu\left(e^{i k \Delta h}+e^{-i k \Delta h}+e^{i l \Delta h}+e^{-i l \Delta h}+e^{i m \Delta h}+e^{-i m \Delta h}-6\right)+(\lambda+\mu)\left(e^{i k \Delta h}+e^{-i k \Delta h}-2\right)}{(\Delta h)^{2}} \\
& A_{y y}=\frac{(\Delta t)^{2}}{\rho} \frac{\mu\left(e^{i k \Delta h}+e^{-i k \Delta h}+e^{i l \Delta h}+e^{-i l \Delta h}+e^{i m \Delta h}+e^{-i m \Delta h}-6\right)+(\lambda+\mu)\left(e^{i l \Delta h}+e^{-i l \Delta h}-2\right)}{(\Delta h)^{2}} \\
& A_{z z}=\frac{(\Delta t)^{2}}{\rho} \frac{\mu\left(e^{i k \Delta h}+e^{-i k \Delta h}+e^{i l \Delta h}+e^{-i l \Delta h}+e^{i m \Delta h}+e^{-i m \Delta h}-6\right)+(\lambda+\mu)\left(e^{i m \Delta h}+e^{-i m \Delta h}-2\right)}{(\Delta h)^{2}} \\
& A_{x y}=A_{y x}=\frac{(\Delta t)^{2}}{\rho} \frac{(\lambda+\mu)\left(e^{i(l+k) \Delta h}+e^{-i(l+k) \Delta h}-e^{i(l-k) \Delta h}-e^{-i(l-k) \Delta h}\right)}{4(\Delta h)^{2}}, \\
& A_{x z}=A_{z x}=\frac{(\Delta t)^{2}}{\rho} \frac{(\lambda+\mu)\left(e^{i(m+k) \Delta h}+e^{-i(m+k) \Delta h}-e^{i(m-k) \Delta h}-e^{-i(m-k) \Delta h}\right)}{4(\Delta h)^{2}} \\
& A_{y z}=A_{z y}=\frac{(\Delta t)^{2}}{\rho} \frac{(\lambda+\mu)\left(e^{i(l+m) \Delta h}+e^{-i(l+m) \Delta h}-e^{i(l-m) \Delta h}-e^{-i(l-m) \Delta h}\right)}{4(\Delta h)^{2}}
\end{aligned}
$$

By defining an auxiliary variable $s_{x y z}^{t}$, Eq. (3-32) can be simplified to the following iterating form:

$$
\begin{aligned}
& \vec{s}_{x y z}^{t+1}-\vec{s}_{x y z}^{t}=A \vec{u}_{x y z}^{t}, \\
& \vec{s}_{x y z}^{t+1}=\vec{u}_{x y z}^{t+1}-\vec{u}_{x y z}^{t} .
\end{aligned}
$$

Therefore, 


$$
\left[\begin{array}{l}
s_{x, x y z}^{t+1} \\
s_{y, x y z}^{t+1} \\
s_{z, x y z}^{t+1} \\
u_{x, x y z}^{t+1} \\
u_{y, x y z}^{t+1} \\
u_{z, x y z}^{t+1}
\end{array}\right]=\left[\begin{array}{cc}
I_{3 \times 3} & A_{3 \times 3} \\
I_{3 \times 3} & I_{3 \times 3}+A_{3 \times 3}
\end{array}\right]\left[\begin{array}{l}
s_{x, x y z}^{t} \\
s_{y, x y z}^{t} \\
s_{z, x y z}^{t} \\
u_{x, x y z}^{t} \\
u_{y, x y z}^{t} \\
u_{z, x y z}^{t}
\end{array}\right]
$$

or:

$$
\left[\begin{array}{l}
\vec{s}_{x y z}^{t+1} \\
\vec{u}_{x y z}^{t+1}
\end{array}\right]=C_{6 \times 6}\left[\begin{array}{l}
\vec{s}_{x y z}^{t} \\
\vec{u}_{x y z}^{t}
\end{array}\right] .
$$

Based on Eq. (3-37), the finite difference simulation is stable if the absolute value of all six eigenvalues of matrix $C_{6 \times 6}$ are smaller than 1 .

$$
\begin{aligned}
& \left|\lambda_{j}\right| \leq 1, \quad j=1, \ldots, 6, \\
& \lambda_{j}=\text { eigenvalue }\left(C_{6 \times 6}\right) .
\end{aligned}
$$

In the simulations, first $\Delta h$ is selected from Eq. (3-27), then $\Delta t$ is selected using Eq. (3-38). Since the eigenvalues could change for different locations in space, two marginal cases are considered where all elements of $A$ are maximum or minimum; and $\Delta t$ is chosen such that Eq. (3-38) remains valid.

The validation of the proposed numerical framework will be discussed in more details in Section 5.1. The application of this framework in simulation of shear wave propagating in soft tissue including a HIFU thermal lesion will be then presented in Section 5.2. 


\section{Chapter 4}

\section{Viscoelasticity Estimation}

In many publications, the cross-correlation of consecutive ultrasound RF signals or ultrasound images is the basis of detecting the longitudinal or shear waves [20], [160]-[162] which results in calculation of time-of-arrival of the waves. In this thesis, the proposed estimation method for detecting the shear wave is based on the combination of Wigner-Ville distribution (WVD) analysis, the alternating sequential filters (ASF) and the circular Hough transform (CHT). It will be demonstrated that the combination of these techniques leads to detection of longitudinal and shear waves generated by an acoustic radiation force impulse at any individual location in the region of interest. This is done to estimate the location of the waves in time; and then to estimate the longitudinal and shear wave parameters such as frequency content, propagation speed and the attenuation coefficient of the medium. Since some cross-term artifacts could exist in the timefrequency profile from the WVD that are not real frequencies [141], [151], specific image processing techniques such as the ASF and $\mathrm{CHT}$ are employed in order to detect all time-frequency points including the real ones and the cross-term artifacts; then, to separate the real time-frequency components from the artificial cross-terms by further analysis. Based on this method, the timefrequency profiles of the local signals are evaluated and the time-of-arrival, and therefore the speed, and frequency of the signal are estimated. Then, by using the Voigt model, the acoustic

properties of the medium are estimated. These estimations are the bases for calculating the 
elasticity and viscosity of the medium and distinguishing between the layers of tissue. The Voigt model is used because it includes both elasticity and viscosity terms and formulates the speed of wave propagation and attenuation coefficient of the material based on viscosity parameters [160]. Therefore, the inverse Voigt model is an appropriate method to estimate the viscoelastic parameters from the estimated speed, frequency, and attenuation coefficient.

The major benefit of the proposed detection and estimation method over the traditional crosscorrelation based methods is its capability of extracting more information from the particle displacement profile. The cross-correlation based methods are normally capable of estimating the time-of-arrival of the wave. On the other hand, the proposed WVD based method provides the instantaneous frequency of the propagating wave as well as its time-of-arrival. This could lead to a more accurate estimation of viscoelastic parameters of the medium.

The other major benefit of the proposed method is its capability of estimating the time-of-arrival of the wave at any spatial point independently without any prior knowledge of the acoustic signal shape at other points. In the correlation-based methods, since the time-of-arrival is estimated by comparing the acoustic signals at two spatial points and calculating the time-shift between them, the calculations are not independent for each point. Therefore, if the calculations fail at the points closer to the source, it will fail at the other farther points. As per the Green's function solution for wave propagation in viscoelastic medium, proposed by Bercoff et al. [56], the formulations show that the shape of the acoustic wave changes during propagation. Although these changes are negligible for two close neighboring points, they could be significant for two far points. This fact increases the chance of correlation-based methods failure in comparison with the proposed timefrequency analysis. This will be further discussed in the next chapter with examples. 


\subsection{Wigner-Ville Distribution of Acoustic Wave}

In this study, the propagating acoustic wave is detected using a discrete version of the WVD given in Eq. (4-1) [141]:

$$
W_{z}[n, k]=2 \operatorname{DFT}_{m \rightarrow k}\left\{z[n+m] z^{*}[n-m]\right\},
$$

where $z[n]$ is the analytical associate of the real signal $s[n]$ at a given local point. $z[n+m]$ and $z[n-$ $m]$ are the $m$-unit shifted versions of the signal to the left and right sides in the time domain, respectively. DFT\{.\} is the discrete Fourier transform with respect to the shift. The WVD of a signal with the length of $N$ is an $N \times N$ matrix of the time-frequency profile that contains the IFs at any time window. As mentioned in Chapter 2, the analytical associate of a real signal is its complex-valued version where the negative frequency components are removed. Using the analytical associate avoids the appearance of some interference terms that are generated from the interaction of positive and negative frequency components without losing any information when dealing with multiple frequency components [141]. The analytical associate of the signal can be derived from the Hilbert transform of the real signal:

$$
z[n]=s[n]+i H\{s(n)\} .
$$

Given the analytical signal $z[n]$, the instantaneous autocorrelation function (IAF) is defined as:

$$
K_{z}[n, m]=z[n+m] z^{*}[n-m] .
$$

The IAF is a function of actual time variable, $n$, and the time lag, $m$. The WVD is defined as the Fourier transform of the IAF with respect to $m$.

In the following, the steps towards the development of the discrete WVD of a signal are summarized:

- Calculation of the Hilbert transform of the real signal,

- Derivation of analytical associate of the signal from the Hilbert transform, 
- Shifting the signal to the right and left side and calculation of the instantaneous autocorrelation function for all possible amounts of lag,

- Implementation of the discrete WVD by calculating the Fourier transform of the IAF.

Therefore, first the IAF of the signal should be calculated for all possible amounts of lag in order to implement the Discrete WVD.

The WVD could be interpreted as the energy distribution of the signal over the TF plane [141]. This method is perfectly localized when dealing with the signals with mono time-frequency component. It has the best resolution among the other time-frequency methods, such as short-time Fourier transform, in the detection of Instantaneous Frequencies [141]. Since WVD is quadratic in nature, it introduces cross terms when dealing with signals with multi time-frequency components. The cross terms are introduced by every pair of time-frequency components in the TF plane. They are located exactly in the middle of the pairs of components.

Since the acoustic displacement profile may contain both longitudinal and shear waves, the WVD could result in generation of cross-terms between the time-frequency components. There are different methods proposed to reduce the cross terms using window-based calculations by smoothing the signal in time and/or in frequency domains in pseudo-WVD and smoothed pseudoWVD methods [9]. These methods, generally, result in reducing the amplitude of cross terms with cost of losing the time-frequency resolution. The goal of this study is to detect the shear wave resulted from ARFI at different locations of the medium. In order to detect longitudinal and shear components, a different approach is introduced by multiplying the WVD result with the Fourier transform of the acoustic signal [141]. In this way, Fourier Transform acts as a window-based filter that eliminates the artificial cross-terms and keeps only the real TF components. The timefrequency profile is then further processed to extract the time-frequency components of the signal 
from the acoustic noise and the cross-terms by utilizing a post-processing algorithm that includes a peak detection method along with morphological operators and the circular Hough transform. The goal is to detect the existing time-frequency points and to eliminate the unwanted cross-terms.

\subsubsection{Morphological Operators}

The morphological gradients has been used along with thresholding in various image processing purposes such as filtering, marking and image segmentation [163]. These operators calculate the difference between the local maximum and minimum values in the neighborhood of the given points in an image using a user defined structuring element (SE). In this project, an algorithm is implemented based on the alternating sequential filters in order to detect and distinguish the boundaries between two instant frequencies in the time-frequency plane. The ASF is a composition of alternating openings and closings with increasingly wide structuring elements. The opening of image $A$ by the structuring element SE is defined as an erosion of $A$ and SE followed by a dilation. The closing of image $A$ and SE is defined as a dilation followed by an erosion. The definitions of the, opening, closing and ASF are presented in equations (4-4) - (4-6), respectively [163].

$$
\begin{gathered}
A \circ S E=(A \ominus S E) \oplus S E, \\
A \bullet S E=(A \oplus S E) \ominus S E \\
A S F^{n}\{A\}=\left(\left(\left(\left(\left(\left(A \bullet S E_{1}\right) \circ S E_{1}\right) \cdot S E_{2}\right) \circ S E_{2}\right) \bullet \ldots\right) \cdot S E_{n}\right) \circ S E_{n},
\end{gathered}
$$

where the operators $\ominus, \oplus$, ० and $\bullet$ are erosion, dilation, opening and closing operators, respectively. In Eq. (4-6), $S E_{1}, S E_{2}, \ldots$ and $S E_{n}$ are the increasingly expanding structuring elements. Therefore, the order of ASF is $n$ and changes accordingly. 
In this work, the TF plane of the acoustic signal at every spatial point as is considered a $2 \mathrm{D}$ image. By utilizing the ASF method, the gaps in areas indicating time-frequency components are removed by using $n=10$ pixels.

\subsubsection{Circular Hough Transform}

The Hough transform is a broadly used image processing technique in shape detection and recognition [164]. This technique is robust to noise and it has been widely used in the recognition of lines, corners, circles, ellipses and any analytically and arbitrary defined object in an image. This method is based on transform mapping and voting rule to detect the best candidate for the defined shape.

In this work, the best candidates of TF components are extracted from the TF plane using circular Hough transform are extracted by searching for the circular shapes with brighter intensities in the TF plane. Then, the frequency and the time-of-travel of the wave are measured at the detected TF points. The amplitude of the signal is also measured to estimate the local attenuation coefficient of the medium.

\subsection{Direct Voigt's Model}

It is possible to write Voigt's model [161] for both longitudinal and shear waves. Based on this model, the speed of the wave and the attenuation coefficient of the medium can be calculated from Eqs. (4-7) - (4-10):

$$
c_{S}=\sqrt{\frac{2\left(\mu^{2}+\omega_{s}^{2} \eta_{s}^{2}\right)}{\rho\left(\mu+\sqrt{\mu^{2}+\omega_{s}^{2} \eta_{s}^{2}}\right)}}
$$




$$
\begin{gathered}
\alpha_{s}=\sqrt{\frac{\rho \omega_{S}^{2}\left(\sqrt{\mu^{2}+\omega_{s}^{2} \eta_{s}^{2}}-\mu\right)}{2\left(\mu^{2}+\omega_{s}^{2} \eta_{s}^{2}\right)}}, \\
c_{p}=\sqrt{\frac{2\left[(\lambda+2 \mu)^{2}+\omega^{2}\left(\eta_{p}+2 \eta_{s}\right)^{2}\right]}{\rho\left[(\lambda+2 \mu)+\sqrt{(\lambda+2 \mu)^{2}+\omega^{2}\left(\eta_{p}+2 \eta_{s}\right)^{2}}\right.},} \\
\alpha_{p}=\sqrt{\frac{\rho \omega^{2}\left(\sqrt{(\lambda+2 \mu)^{2}+\omega^{2}\left(\eta_{p}+2 \eta_{s}\right)^{2}}-(\lambda+2 \mu)\right.}{2\left((\lambda+2 \mu)^{2}+\omega^{2}\left(\eta_{p}+2 \eta_{s}\right)^{2}\right)}}
\end{gathered}
$$

where $\omega$ and $\omega_{s}$ are the angular frequencies of the longitudinal and shear waves, respectively. In this study, an inverse version of the Voigt's equations is utilized.

\subsection{Inverse Voigt's Model}

The shear modulus and shear viscosity are estimated by approximating the frequency, attenuation, and speed of propagation of the waves. Equations (4-11) and (4-12) show the local shear modulus and viscosity terms based on the local shear speed, frequency and attenuation:

$$
\begin{gathered}
\mu=\frac{\rho c_{S}^{2} \omega_{S}^{2}\left(\omega_{S}^{2}-\alpha_{s}^{2} c_{S}^{2}\right)}{\left(\omega_{s}^{2}+\alpha_{s}^{2} c_{S}^{2}\right)^{2}}, \\
\eta_{S}=\frac{2 \rho c_{S}^{3} \omega_{S}^{2} \alpha_{S}}{\left(\omega_{S}^{2}+\alpha_{S}^{2} c_{S}^{2}\right)^{2}} .
\end{gathered}
$$

In case of very high frequencies when $\omega_{s} \rightarrow \infty$, these equations can be approximated as $\mu=\rho c_{s}^{2}$ and $\eta_{s}=0$. Since the frequency of shear wave generated in tissue-like medium is in low range of $100-1000 \mathrm{~Hz}$, this assumption is not accurate. Therefore, using the frequency and attenuation coefficient information leads to a more accurate estimation of the viscoelastic parameters.

The results of the proposed detection method will be discussed in Section 5.3. This method will be applied on simulated data of a shear wave propagation in a HIFU lesion located in the center of 
normal tissue. It will be discussed that the proposed time-frequency algorithm can detect shear wave at every location in the simulation. It can also estimate the time-of-flight of the wave and its center frequency. The estimations for every single local point are independent to the other surrounding points. In Sections 5.3 and 5.4, the results of shear elasticity and shear viscosity will be shown and discussed. 


\section{Chapter 5}

\section{Results and Discussions}

In this chapter, the results from the acoustic wave propagation simulation are presented and validated by comparing them with the published results. Then the result of the proposed estimation method is demonstrated in finding the local acoustic parameters of a simulated HIFU thermal lesion induced inside a normal tissue.

In Sections 5.1 and 5.2, the results of the proposed method in Chapter 3 are presented. First the numerical method is validated by comparing its results with other studies. Then the acoustic wave propagation in simulated HIFU lesions is presented and discusses.

In Section 5.3, the results of the proposed detection method in Chapter 4 are shown. In this section, the estimations of time-of-arrival, center frequency and amplitude of simulated shear wave in a soft tissue with ellipsoid HIFU lesion are discussed. Then the estimation of shear elasticity and viscosity is explained. In the discussion section, it is illustrated that the proposed detection method can differentiate between normal tissue and HIFU lesion.

\subsection{Validation of the Simulation}

This section shows the results of the proposed numerical framework in Chapter 3 along with its validation by comparing its results with other research works. In order to validate the proposed 
simulations approach, two published simulation studies are considered. In the first comparison, the proposed simulation method including the Perfectly Matched Layer is compared with the results from an analytical solution by Bercoff et al. in homogeneous media [56]. In the second comparison, the validation is based on the simulation by Yohann Dudouit [157] in a multilayer media in seismologic studies.

\subsubsection{Comparison with Green's Function Method}

Bercoff et al. [56] proposed a solution to the generalized Navier's equation in an infinite homogeneous isotropic elastic and viscous medium using Green's functions. In this study, Green's function has been used to formulate the particle displacement of the medium corresponding to an impulse spatio-temporal excitation. The proposed analytical solution by Bercoff et al. includes three terms that represent bulk component, shear component and the coupling term between the bulk and shear components, as given in Eqs. (5-1) - (5-4) [56]:

$$
\begin{gathered}
g_{i j}(\vec{r}, t)=a_{i}\left(g_{i j}^{p}(\vec{r}, t)+g_{i j}^{S}(\vec{r}, t)+g_{i j}^{p s}(\vec{r}, t)\right), \\
g_{i j}^{p}(\vec{r}, t)=\frac{1}{4 \pi \rho c_{p}} \frac{1}{\sqrt{2 \pi v_{p} t}} \gamma_{i} \gamma_{j} \frac{1}{r} e^{-\frac{\left(t-\frac{r}{c_{p}}\right)^{2} c_{p}^{2}}{2 v_{p} t}}, \\
g_{i j}^{S}(\vec{r}, t)=\frac{1}{4 \pi \rho c_{s}} \frac{1}{\sqrt{2 \pi v_{s} t}} \frac{\delta_{i j}-\gamma_{i} \gamma_{j}}{r} e^{-\frac{\left(t-\frac{r}{c_{S}}\right)^{2} c_{S}^{2}}{2 v_{s} t}}, \\
g_{i j}^{p s}(\vec{r}, t)=\frac{1}{4 \pi \rho} \frac{3 \gamma_{i} \gamma_{j}-\delta_{i j}}{r^{3}}\left[I_{p}(\vec{r}, t)+I_{s}(\vec{r}, t)\right],
\end{gathered}
$$

where,

$$
I_{p}(\vec{r}, t)=\frac{\sqrt{v_{p} t}}{\sqrt{2 \pi} c_{p}}\left[e^{-\frac{t^{2} c_{p}^{2}}{2 v_{p} t}}-e^{-\frac{\left(t-\frac{r}{c_{p}}\right)^{2} c_{p}^{2}}{2 v_{p} t}}\right]+\frac{t}{2}\left[\operatorname{Erf}\left(\frac{c_{p} t}{\sqrt{2 v_{p} t}}\right)-\operatorname{Erf}\left(\frac{c_{p}\left(t-\frac{r}{c_{p}}\right)}{\sqrt{2 v_{p} t}}\right)\right],
$$




$$
I_{S}(\vec{r}, t)=\frac{\sqrt{v_{s} t}}{\sqrt{2 \pi} c_{S}}\left[e^{-\frac{t^{2} c_{S}^{2}}{2 v_{s} t}}-e^{-\frac{\left(t-\frac{r}{c_{S}}\right)^{2} c_{S}^{2}}{2 v_{s} t}}\right]+\frac{t}{2}\left[\operatorname{Erf}\left(\frac{c_{s} t}{\sqrt{2 v_{s} t}}\right)-\operatorname{Erf}\left(\frac{c_{s}\left(t-\frac{r}{c_{S}}\right)}{\sqrt{2 v_{s} t}}\right)\right] .
$$

In the first simulation, the results from the proposed finite difference method are compared with the results from the Bercoff's formulations in a homogeneous viscoelastic medium with the properties given in Table 5.1. The parameters in this simulation are not representative of a typical soft tissue. The speed of longitudinal wave chosen in this simulation is $40 \mathrm{~m} / \mathrm{s}$ much smaller than $1500 \mathrm{~m} / \mathrm{s}$ in a typical soft tissue. The reason of using these parameters is to show longitudinal and shear waves in a single simulation [56].

Table 5.1: The specifications of Simulation 1

\begin{tabular}{|l|l|l|}
\hline \multirow{5}{*}{ Mechanical Properties } & Density & $\rho=1,000 \mathrm{~kg} / \mathrm{m}^{3}$ \\
& Lame's first parameter & $\lambda=1,598 \mathrm{kPa}$ \\
& Lame's second parameter & $\mu=1 \mathrm{kPa}$ \\
& Bulk Viscosity & $v_{p}=0.2 \mathrm{~Pa} . \mathrm{s}$ \\
& Shear Viscosity & $v_{s}=0.2 \mathrm{~Pa} . \mathrm{s}$ \\
\hline \multirow{5}{*}{ Geometry } & $-20 \mathrm{~mm} \leq x \leq 20 \mathrm{~mm}$ & $d x=1 \mathrm{~mm}$ \\
& $-20 \mathrm{~mm} \leq y \leq 20 \mathrm{~mm}$ & $d y=1 \mathrm{~mm}$ \\
Simulation Duration & $-20 \mathrm{~mm} \leq z \leq 20 \mathrm{~mm}$ & $d z=1 \mathrm{~mm}$ \\
\hline \multirow{3}{*}{ PML Parameters } & $0 \leq t \leq 15 \mathrm{~ms}$ & $d t=100 \mathrm{~ns}$ \\
& PML Length & $d=3 \mathrm{~mm}$ \\
& PML Order & $n=2$ \\
& Reflection Coefficient & $R_{j}=10^{-3}$ \\
\hline
\end{tabular}




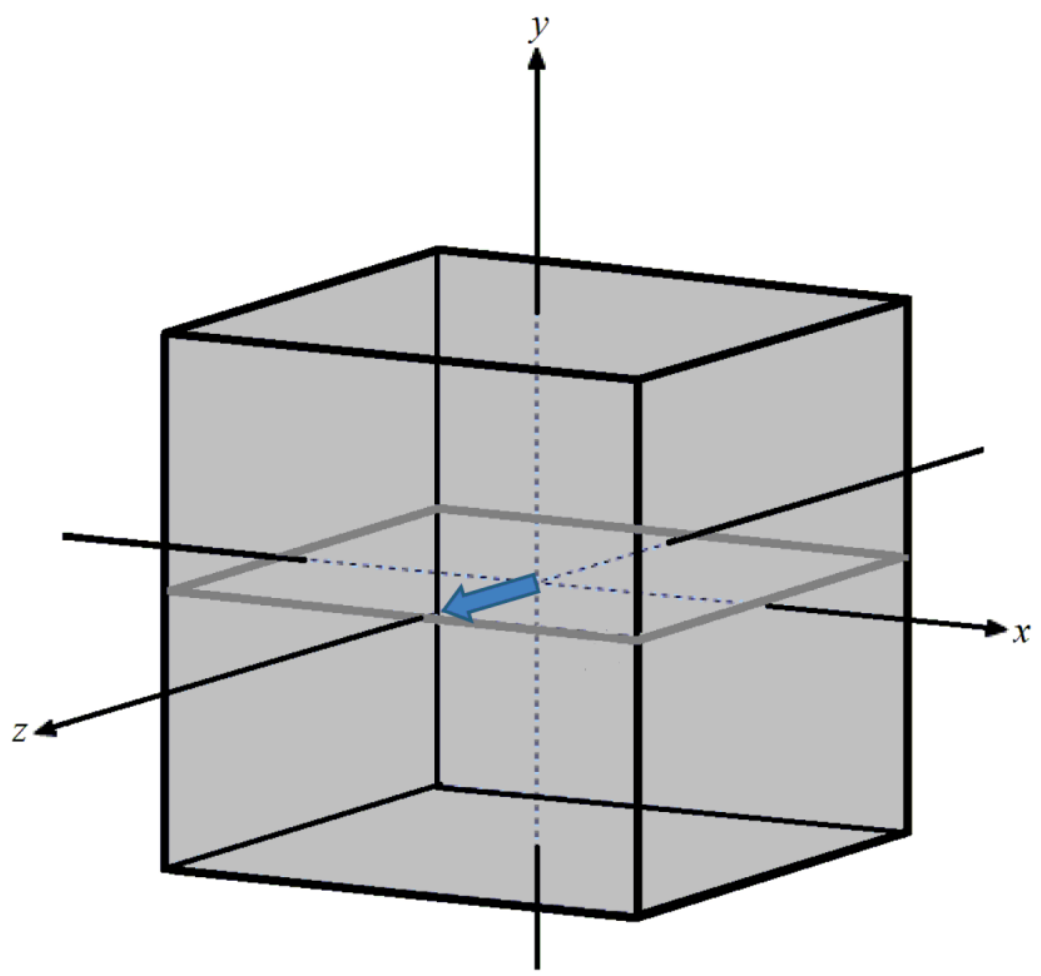

Figure 5.1: The geometry and the source in the first simulation validation.

In this simulation, the ultrasound source is modeled as an impulse at the origin in $z$ direction, and the particle displacement is calculated in different locations. The simulation geometry and the source are illustrated in Figure 5.1.

The results of the simulations are shown in Figure 5.2 at two different radial points. 


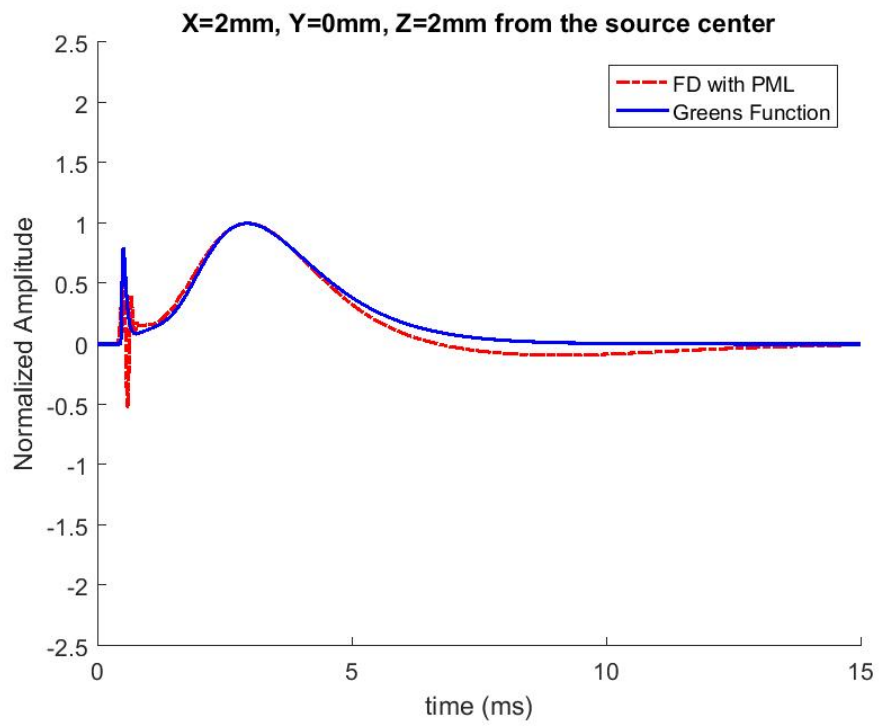

(a)

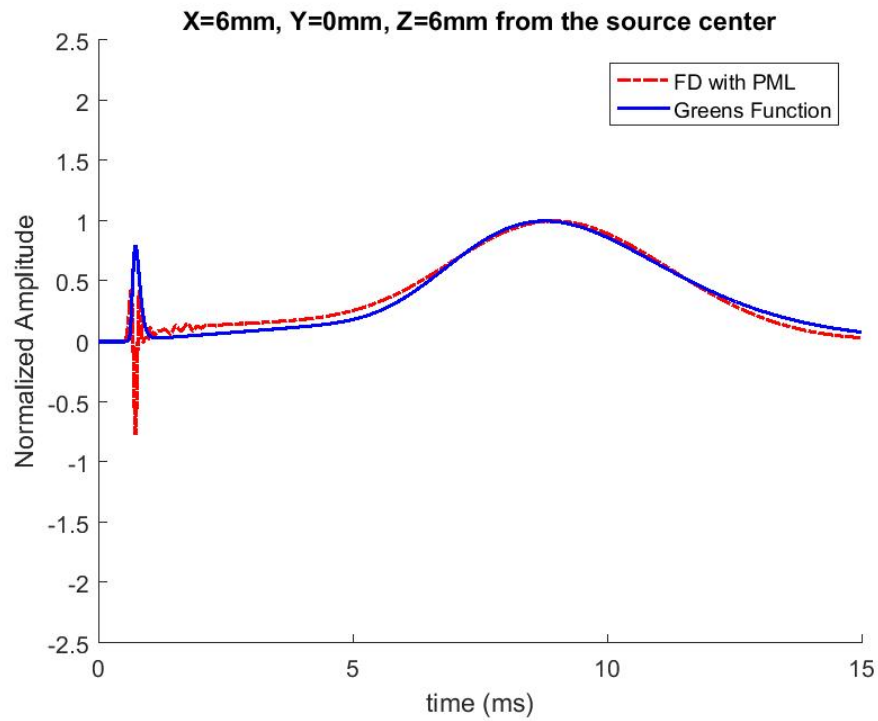

(b)

Figure 5.2: Comparison between the results of the Green's functions formulation (Bercoff et al. [56]) and the proposed finite difference method using PML at two different radial points.

The results of the simulations are also shown in Figure 5.3 at one axial and one lateral points. 


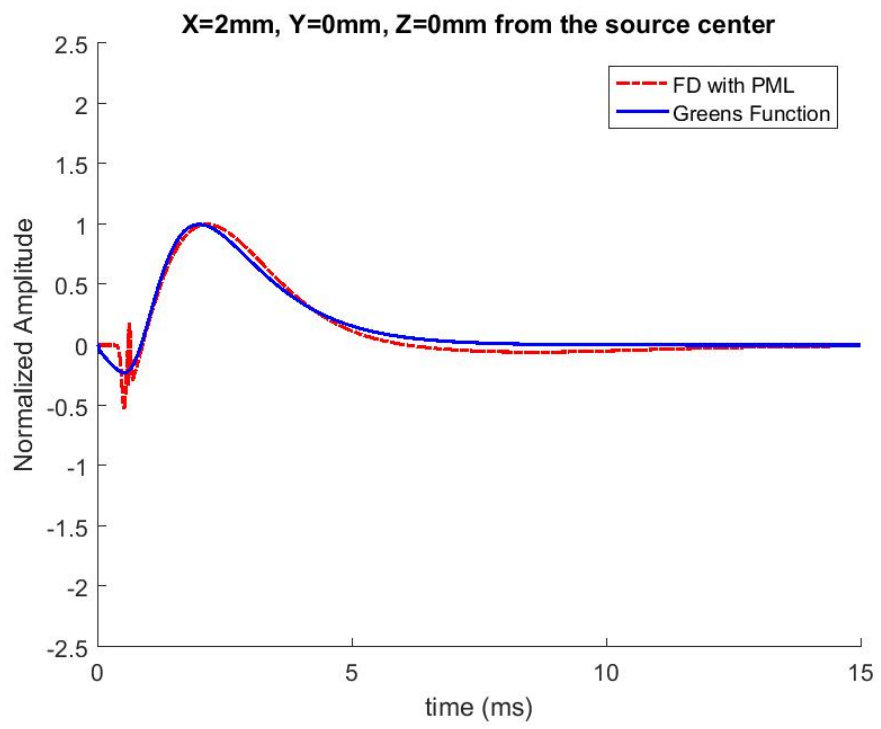

(a)

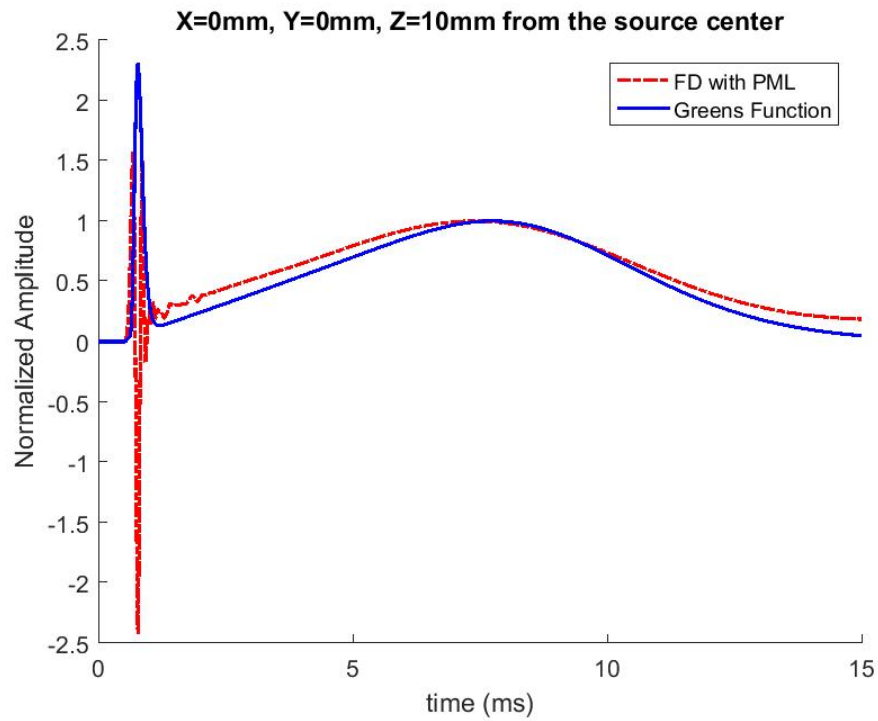

(b)

Figure 5.3: Comparison between the results of the Green's functions formulation (Bercoff et al. [56]) and the proposed finite difference method using PML at (a) a lateral point, and (b) an axial point.

The effect of PML has been illustrated in Figure 5.4 and Figure 5.5 for the same locations. These figures show how the PML can efficiently remove the reflected waves from computational boundaries. 


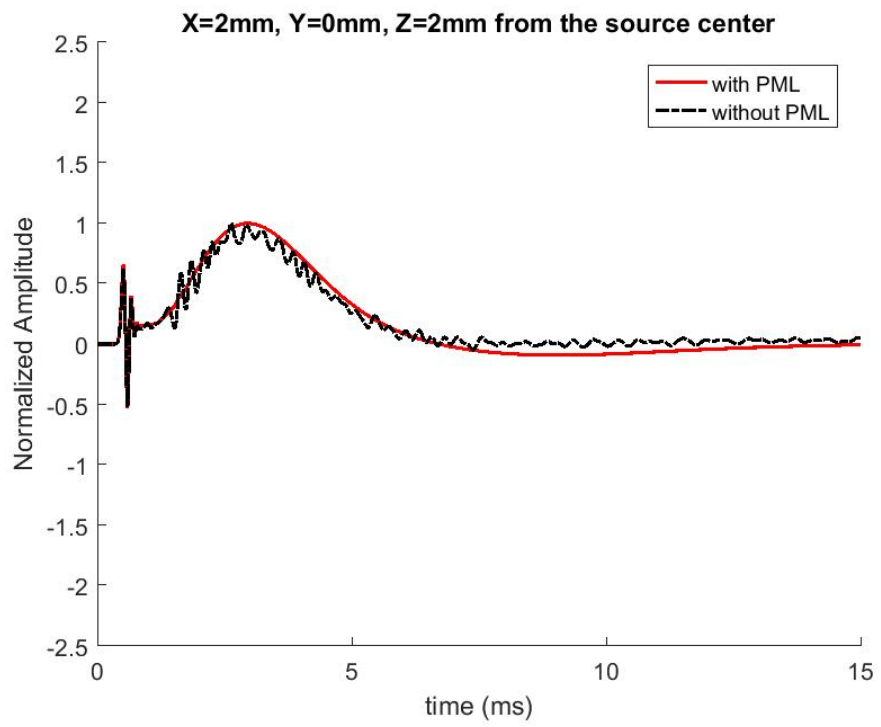

(a)

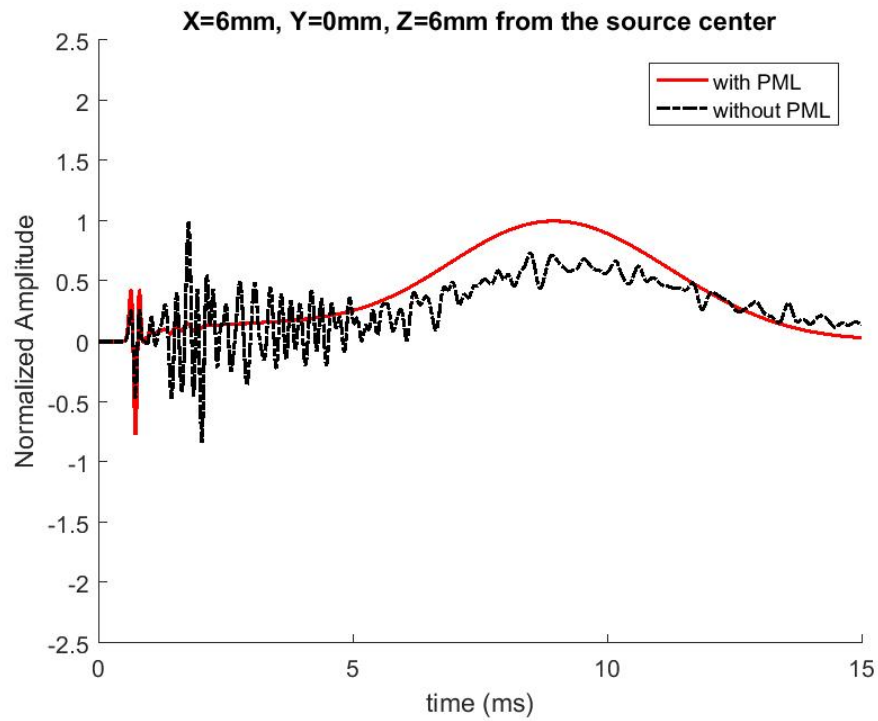

(b)

Figure 5.4: The simulation results with and without PML at two radial points. 


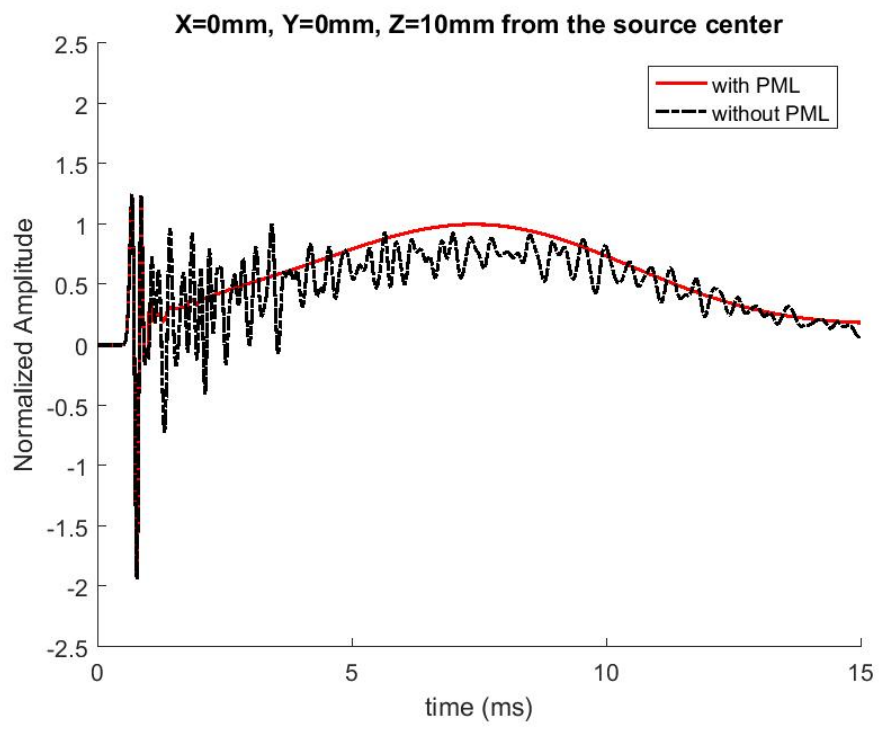

(a)

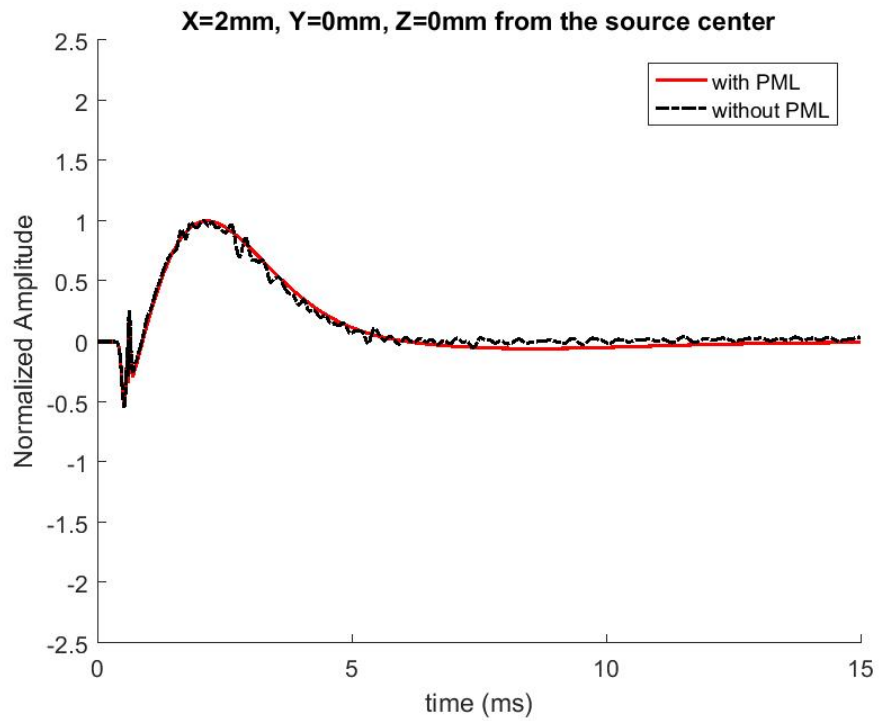

(b)

Figure 5.5: The simulation results with and without PML at (a) a lateral point, and (b) an axial point.

It should be noted that the shear wave amplitude drops in the results without PML when the spatial point is closer to the boundaries, e.g. Figure 5.4 (b) and Figure 5.5 (a). This is due to the numerical reflections of the shear wave from the boundaries which are all eliminated after using PML. 
The total acoustic energy as computed over the entire geometry is also shown in Figure 5.6 for the simulations with and without PML. This figure also depicts that the energy is attenuated in the PML region.

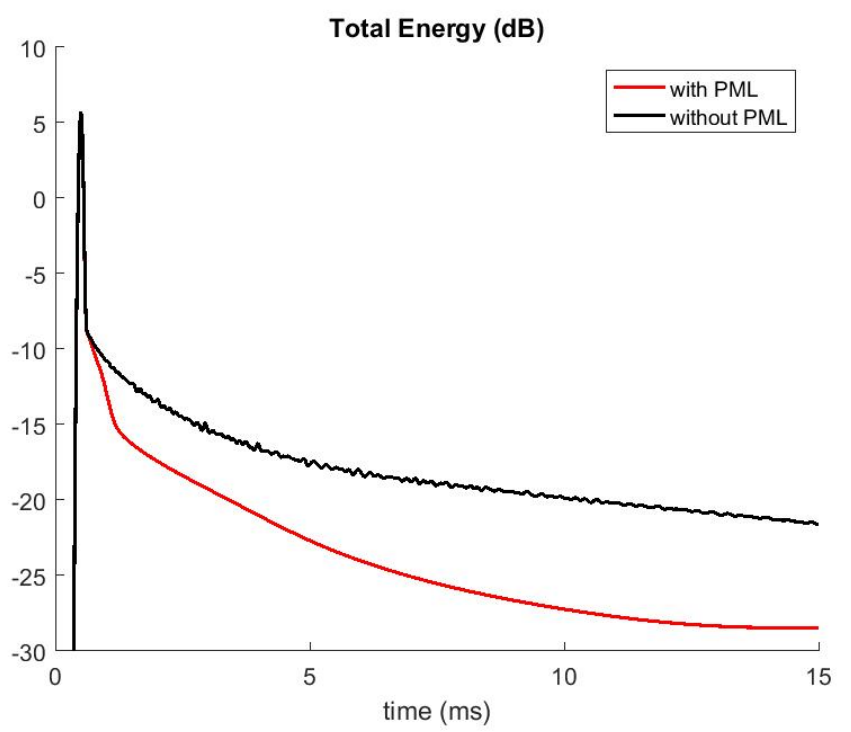

Figure 5.6: The comparison of total energy attenuation in time (in decibels) with/without PML.

As illustrated in the above figures, the results when applying PML are in very good agreements with the results from Bercoff's formulation.

\subsubsection{Two-Layer Large Scale Medium Simulation}

In this section, the simulation results of a large size viscoelastic medium, representing a seismological application, has been provided and compared with the results from Dudouit studies [157].

In this simulation, a two-layer medium has been simulated with the characteristics in Table 5.2. 
Both simulations used a source function consisting of a second order Ricker function, $h(t)$ and a Gaussian regularized Dirac function $g\left(\left|r-r_{s}\right|\right)$ over a sphere located at $r_{s}$ whose radius is $r_{0}$. This source function is given by:

$$
f(r, t)=h(t) g\left(\left|r-r_{s}\right|\right) \frac{\overrightarrow{r-r_{s}}}{\left|r-r_{s}\right|}
$$

where the Ricker wavelet function, whose peak frequency is $f_{0}$, is given by:

$$
h(t)=\left(2 \pi^{2}\left(f_{0} t-1\right)^{2}-1\right) e^{-\pi^{2}\left(f_{0} t-1\right)^{2}},
$$

and the regularized Dirac function is

$$
g\left(\left|r-r_{s}\right|\right)=e^{-7 \frac{\left|r-r_{s}\right|^{2}}{r_{0}^{2}}} / r_{0}^{2}
$$

In Eq. (5-7) the vector term $\frac{\overrightarrow{r-r_{s}}}{\left|r-r_{s}\right|}$ generates the applied acoustic displacement in the radial direction, which implies that the source has only longitudinal term in the layer containing the origin. The source in this simulation was at location $(x=100, y=0, z=100) \mathrm{m}$; moreover, in both simulations $f_{0}$ was taken to be $40 \mathrm{~Hz}$, a typical frequency used in seismology applications. The results from Dudouit studies, as shown in Figure 2.11 of his thesis [157], and this simulation are shown in Figure 5.7. This figure shows how the wave propagates from the first layer to the second one. It should be noted that the physical domain geometry in this simulation is the same as that given by Dudouit; however, the PML length and parameters are different. The wave source is in the first layer and generates a pure longitudinal wave.

As shown in Figure 5.7, in both simulations the propagating wave is initially only longitudinal wave. The shear wave is then created after the wave propagates toward the interface between the layers. Both simulations show that the shear wave is generated and propagates in the same manner. 

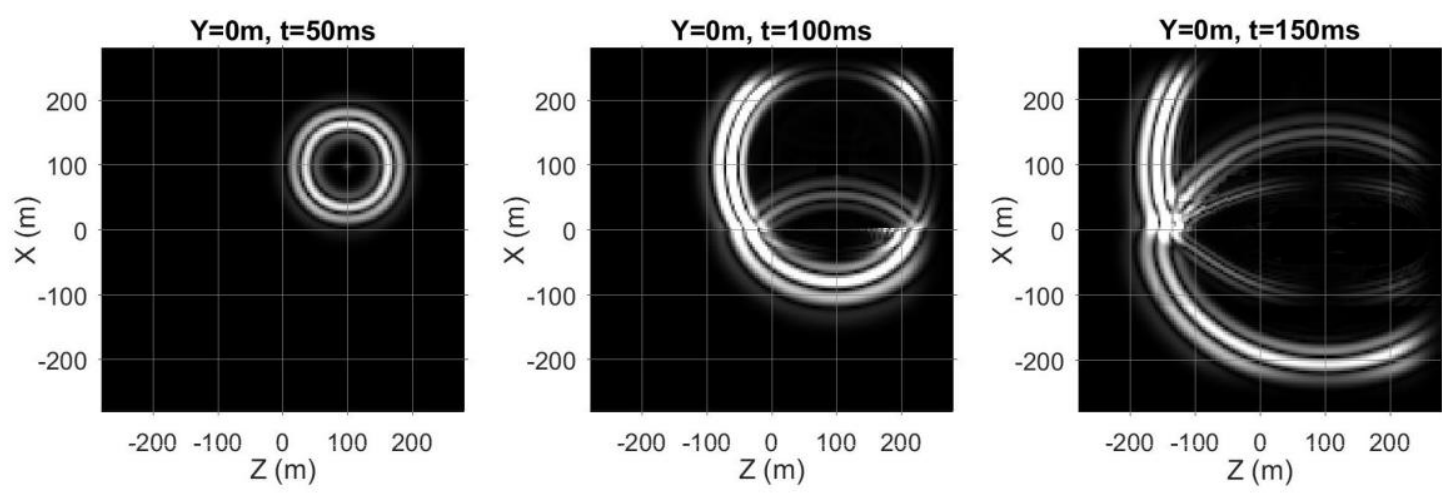

(a)
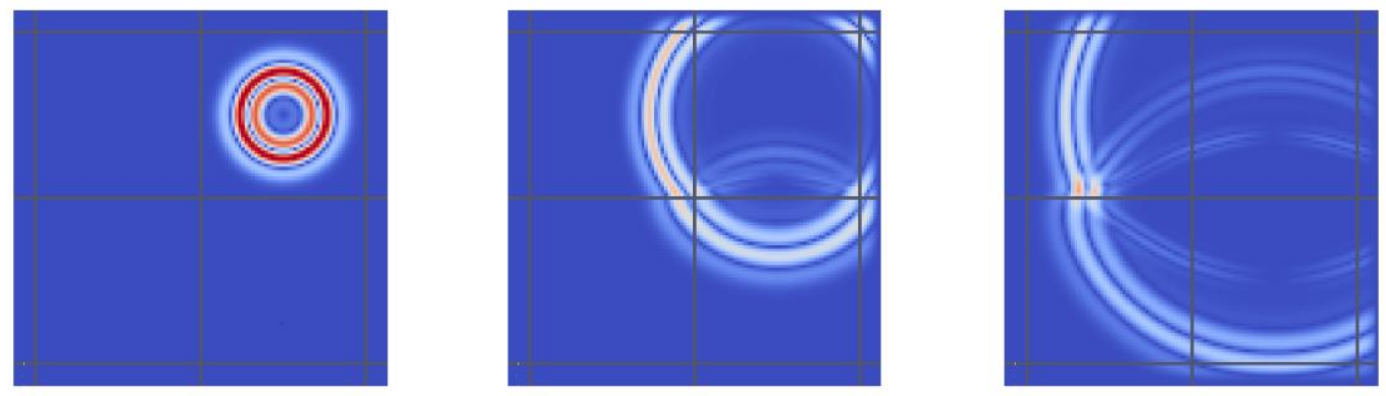

(b)

Figure 5.7: Comparison between results from (a) the proposed method, and (b) the results from Dudouit studies [157] at $t=50 \mathrm{~ms}, t=100 \mathrm{~ms}$ and $\mathrm{t}=150 \mathrm{~ms}$.

Table 5.2: The specifications of Simulation 2

\begin{tabular}{|c|c|c|c|}
\hline & & Layer 1 & Layer 2 \\
\hline Mechanical Properties & $\begin{array}{l}\text { Density } \\
\text { Lame's } 1^{\text {st }} \text { parameter } \\
\text { Lame's } 2^{\text {nd }} \text { parameter } \\
\text { Bulk Viscosity } \\
\text { Shear Viscosity }\end{array}$ & $\begin{array}{c}\rho=2,100 \mathrm{~kg} / \mathrm{m}^{3} \\
\lambda=4.2 \times 10^{9} \mathrm{~Pa} \\
\mu=2.1 \times 10^{9} \mathrm{~Pa} \\
v_{p}=0 \text { Pa.s } \\
v_{s}=0 \text { Pa.s }\end{array}$ & $\begin{array}{c}\rho=2,300 \mathrm{~kg} / \mathrm{m}^{3} \\
\lambda=7.774 \times 10^{9} \mathrm{~Pa} \\
\mu=3.887 \times 10^{9} \mathrm{~Pa} \\
v_{p}=0 \text { Pa.s } \\
v_{s}=0 \text { Pa.s }\end{array}$ \\
\hline Geometry & $\begin{aligned} d x & =4 \mathrm{~m} \\
d y & =4 \mathrm{~m} \\
d z & =4 \mathrm{~m}\end{aligned}$ & $\begin{array}{c}0 \leq x \leq 280 \mathrm{~m} \\
-280 \mathrm{~m} \leq y \leq 280 \mathrm{~m} \\
-280 \mathrm{~m} \leq z \leq 280 \mathrm{~m}\end{array}$ & $\begin{array}{c}-280 \mathrm{~m} \leq x \leq 0 \\
-280 \mathrm{~m} \leq y \leq 280 \mathrm{~m} \\
-280 \mathrm{~m} \leq z \leq 280 \mathrm{~m}\end{array}$ \\
\hline Simulation Duration & $d t=500 \mu \mathrm{s}$ & \multicolumn{2}{|c|}{$0 \leq t \leq 150 \mathrm{~ms}$} \\
\hline PML Parameters & $\begin{array}{l}\text { PML Length } \\
\text { PML Order } \\
\text { Reflection Coefficient }\end{array}$ & \multicolumn{2}{|c|}{$\begin{array}{c}d=80 \mathrm{~m} \\
n=1 \text { (Linear) } \\
R_{j}=10^{-4}\end{array}$} \\
\hline
\end{tabular}




\subsection{Simulations of Acoustic Wave Propagation in HIFU Lesion}

In this section, an application of the proposed numerical model in the field of therapeutic biomedical ultrasound is studied. As it was discussed in Chapter 2, High Intensity Focused Ultrasound (HIFU) is a minimally-invasive therapeutic approach that has been employed to destroy tumors locally by inducing localized heat inside tumors and increasing their temperature approximately to above $60^{\circ} \mathrm{C}$ [165], [166]. In the HIFU therapy, the high intensity ultrasound beam is focused and delivered to the targeted tissue and the ultrasound mechanical energy is converted into heat energy through tissue absorption. Consequently, the temperature of tissue is raised locally to the levels that irreversible structural changes are resulted at that location leading to tissue coagulation necrosis. It has been demonstrated that the mechanical properties of coagulated tissue, a.k.a. a thermal lesion, are different than the surrounding normal tissue [54], [167].

In the following sections, the propagation of ultrasound wave is simulated within a tissue-like medium with a HIFU thermal lesion induced in the middle region of the tissue.

\subsubsection{Double Indentation Experiment}

The HIFU lesion simulation parameters are based on the double indentation measurements [168] on samples from chicken breast tissue. First, a large HIFU thermal lesion was induced; then, the density, Young's and Shear moduli of three samples from the normal tissue and three samples from the HIFU thermal lesion area were measured. Young's and shear moduli were measured based on the method proposed by Hayes et al. [168] using Mach-1 ${ }^{\mathrm{TM}}$ mechanical tester (Biomomentum Inc., Laval, QC, Canada). The tester has a vertical and horizontal stage, so it can perform compression, tension, and shear tests. In double indentation method, two spherical indenters with different radii are used to compress the tissue; then, two graphs for the 
parameter under test are plotted as functions of load factor for the spherical indenters. The overlap between these two graphs can result in estimation of Young's modulus, shear modulus, and Poisson's ratio.

In these measurements, the diameters of the indenters were $4 \mathrm{~mm}$ and $8 \mathrm{~mm}$. The samples were cut wide enough; therefore, the indenters could be placed far from their edges to have more accurate measurements. The thickness of the samples was between $3 \mathrm{~mm}$ to $4 \mathrm{~mm}$ and they were placed on a rigid support. The maximum indentation load was 100 grams and the measurements were captured from the linear part of the force-position curve. These measured Young's modulus and shear modulus parameters can be converted into Lame's first and second parameters.

In Figure 5.8, the HIFU treatment setup, including the HIFU transducer and the tissue holder, and two chicken breast samples are shown. The induced HIFU lesions in the chicken breast sample are visible in this figure.

These lesions are formed after long HIFU treatments with the peak-to-peak pressure of 3.5 MPa at the focal region, center frequency of $1 \mathrm{MHz}, 50 \%$ duty cycle and the duration of 10 minutes. In Figure 5.9 (a) some samples are shown which are cut from the HIFU lesions and the normal tissue. Figure 5.9 (b) illustrates Mach- $1^{\mathrm{TM}}$ mechanical tester during a double indentation measurement. 


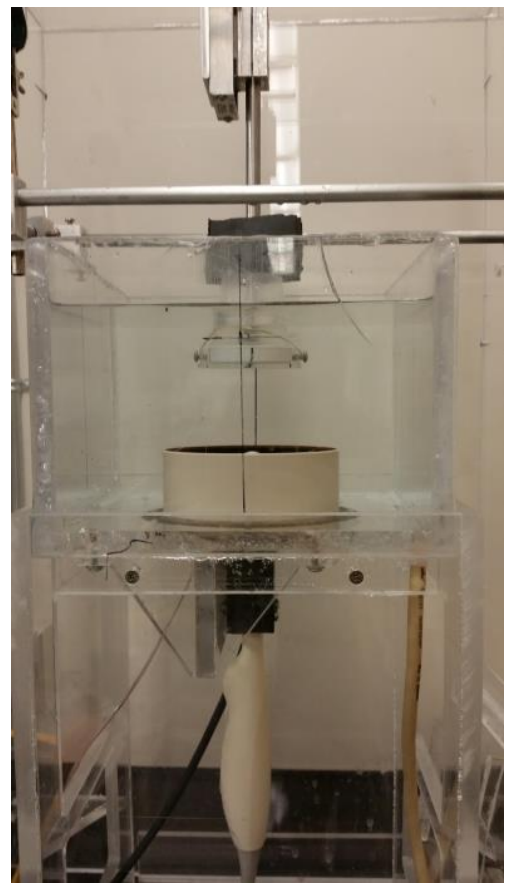

(a)

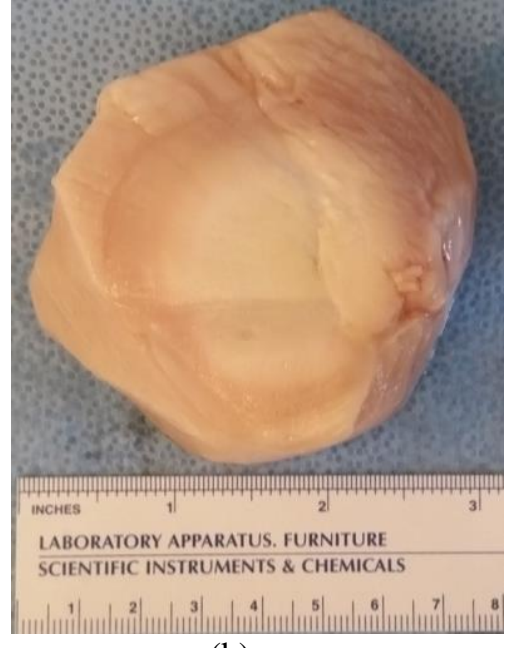

(b)

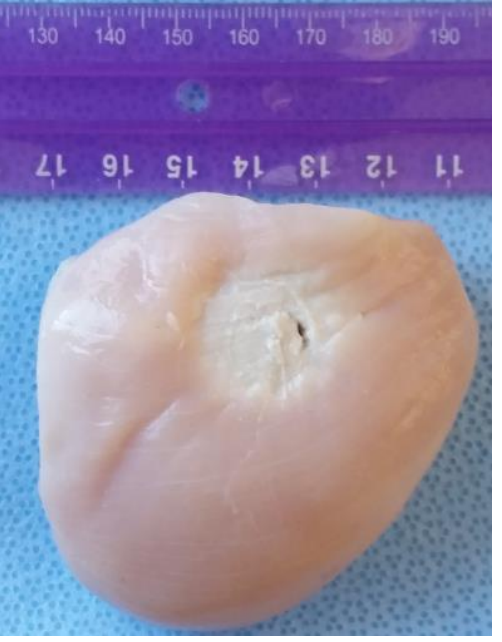

(c)

Figure 5.8: (a) HIFU treatment setup, (b) and (c) two pieces of chicken breast with induced HIFU lesions. 
Table 5.3 shows the resulted mechanical parameters from the double indentation tests:

Table 5.3: The results from double indentation experiment

\begin{tabular}{|l|llll|}
\hline \multirow{2}{*}{$\begin{array}{l}\text { Mechanical } \\
\text { Properties }\end{array}$} & Normal Tissue: & $\rho=1,004 \mathrm{~kg} / \mathrm{m}^{3}$ & $\lambda=2.58 \times 10^{9} \mathrm{~Pa}$ & $\mu=3.27 \times 10^{3} \mathrm{~Pa}$ \\
\cline { 2 - 5 } & HIFU lesion: & $\rho=1,104 \mathrm{~kg} / \mathrm{m}^{3}$ & $\lambda=2.72 \times 10^{9} \mathrm{~Pa}$ & $\mu=11.74 \times 10^{3} \mathrm{~Pa}$ \\
\hline
\end{tabular}

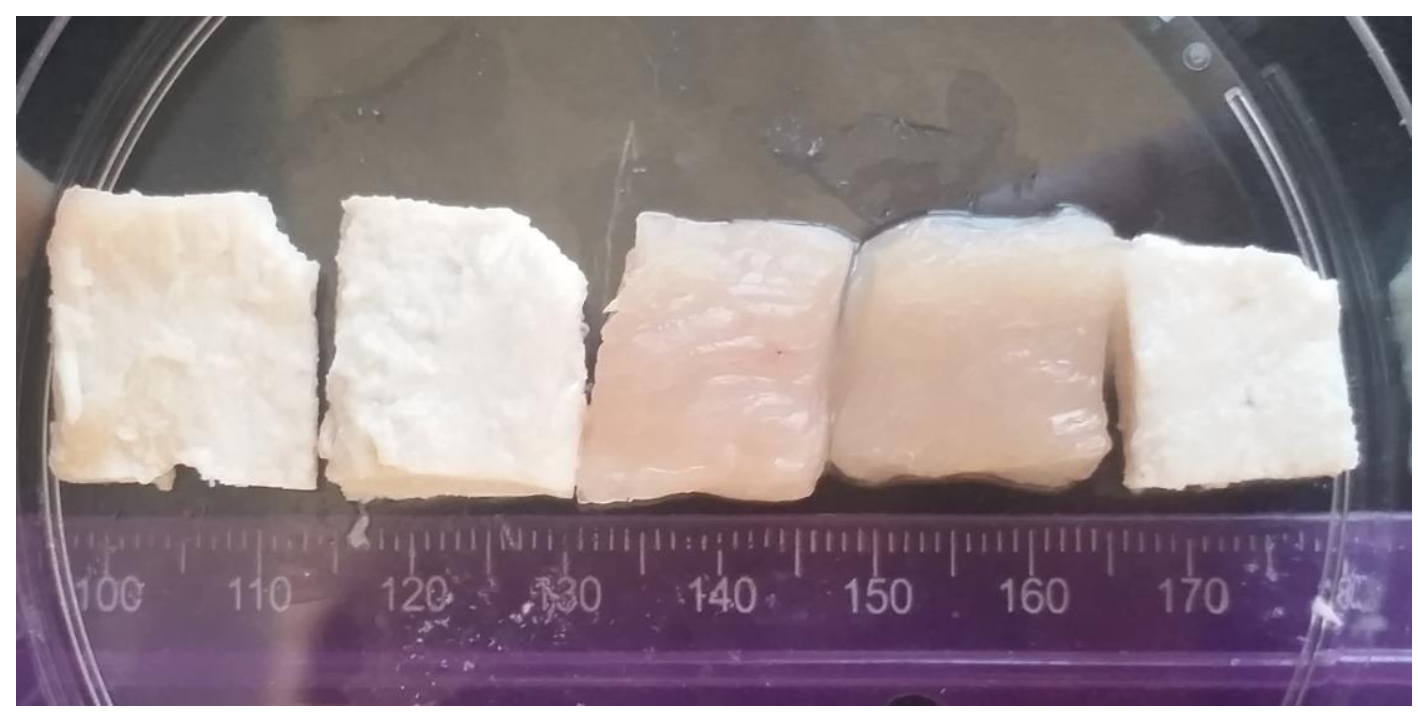

(a)

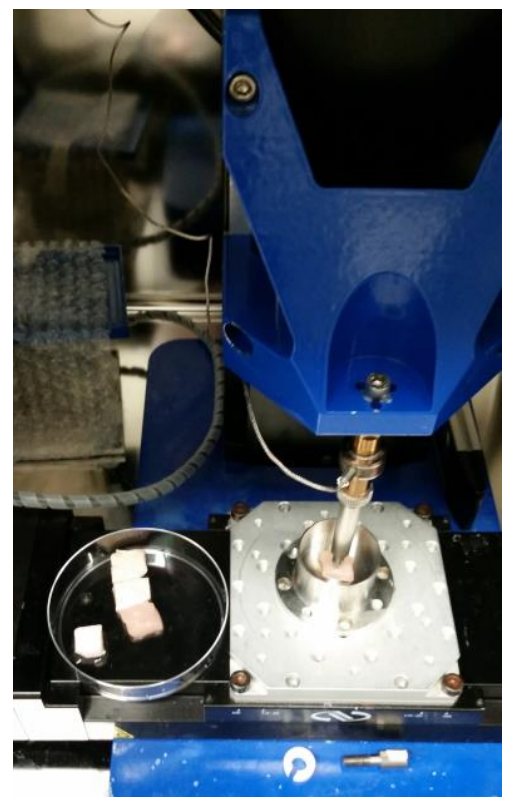

(b)

Figure 5.9: (a) Samples from HIFU induces lesions and the normal chicken breast tissue, (b) Mach-1 ${ }^{\mathrm{TM}}$ mechanical tester during double indentation measurement 


\subsubsection{Ultrasound Shear-Wave Simulation in Cubic Inhomogeneous Tissue}

As illustrated in Figure 5.10, a cubic HIFU lesion, as a simplified lesion geometry, is defined at the center of normal tissue in this simulation. An ARFI is used as the source of shear wave with the duration of $50 \mu$ s located in the center of the cubic HIFU lesion. The Lame's first and second coefficients and the density values used for the normal chicken breast and the thermally induced HIFU lesion are from the double indentation measurements in Table 5.3.

In this simulation, the bulk and shear viscosities are set as 10 Pa.s and $1 \mathrm{~Pa}$.s, respectively. The reason of choosing these values is base on the fact that the bulk and shear viscosities of soft tissue are usually in the range of 1-10 Pa.s [169], and the bulk viscosity is normally larger than shear viscosity. The simulation parameters are summarized in Table 5.4. This table also shows the geometry of the simulated area, and the PML parameters.

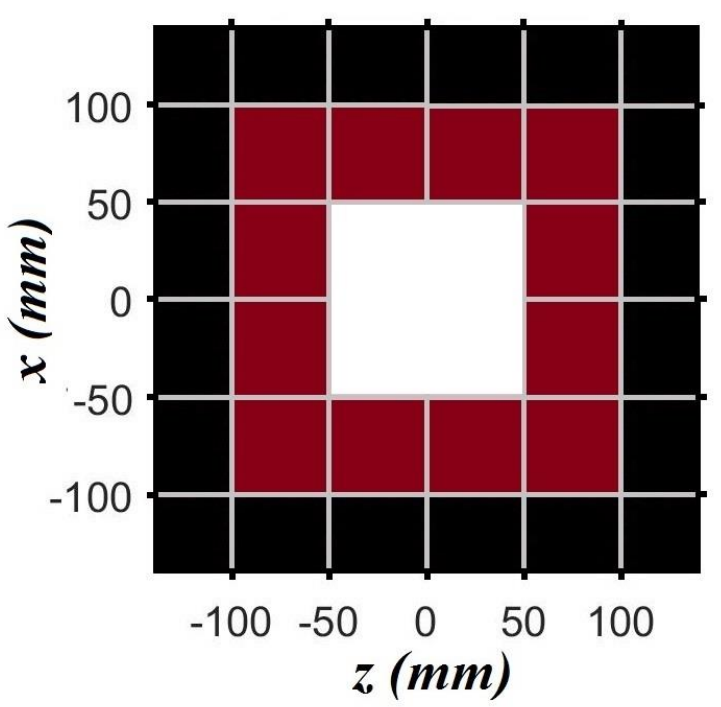

Figure 5.10. The simulation geometry: thermal HIFU lesion is inside the normal tissue, and the black area is the PML region 
Table 5.4: The specifications of Simulation 3

\begin{tabular}{|c|c|c|c|}
\hline & & Normal Tissue & HIFU lesion \\
\hline $\begin{array}{l}\text { Mechanical } \\
\text { Properties (derived } \\
\text { from measurements) }\end{array}$ & $\begin{array}{l}\text { Density } \\
\text { Lame's } 1^{\text {st }} \text { parameter } \\
\text { Lame's } 2^{\text {nd }} \text { parameter } \\
\text { Bulk Viscosity } \\
\text { Shear Viscosity }\end{array}$ & $\begin{array}{c}\rho=1,004 \mathrm{~kg} / \mathrm{m}^{3} \\
\lambda=2.58 \times 10^{9} \mathrm{~Pa} \\
\mu=3.27 \times 10^{3} \mathrm{~Pa} \\
\nu_{\mathrm{p}}=10 \text { Pa.s } \\
\nu_{\mathrm{s}}=1 \text { Pa.s }\end{array}$ & $\begin{array}{c}\rho=1,104 \mathrm{~kg} / \mathrm{m}^{3} \\
\lambda=2.72 \times 10^{9} \mathrm{~Pa} \\
\mu=11.74 \times 10^{3} \mathrm{~Pa} \\
v_{\mathrm{p}}=10 \mathrm{~Pa} . \mathrm{s} \\
v_{\mathrm{s}}=1 \mathrm{~Pa} . \mathrm{s}\end{array}$ \\
\hline Geometry & $\begin{aligned} \mathrm{dx} & =2 \mathrm{~mm} \\
\mathrm{dy} & =2 \mathrm{~mm} \\
\mathrm{dz} & =2 \mathrm{~mm}\end{aligned}$ & $\begin{array}{l}-14 \mathrm{~cm} \leq \mathrm{x} \leq 14 \mathrm{~cm} \\
-14 \mathrm{~cm} \leq \mathrm{y} \leq 14 \mathrm{~cm} \\
-14 \mathrm{~cm} \leq \mathrm{z} \leq 14 \mathrm{~cm}\end{array}$ & $\begin{array}{l}-5 \mathrm{~cm} \leq \mathrm{x} \leq 5 \mathrm{~cm} \\
-5 \mathrm{~cm} \leq \mathrm{y} \leq 5 \mathrm{~cm} \\
-5 \mathrm{~cm} \leq \mathrm{z} \leq 5 \mathrm{~cm}\end{array}$ \\
\hline Simulation Duration & $\mathrm{dt}=0.25 \mathrm{~ms}$ & \multicolumn{2}{|c|}{$0 \leq \mathrm{t} \leq 150 \mathrm{~ms}$} \\
\hline PML Parameters & $\begin{array}{l}\text { PML Length } \\
\text { PML Order } \\
\text { Reflection Coefficient }\end{array}$ & \multicolumn{2}{|c|}{$\begin{array}{c}\mathrm{d}=4 \mathrm{~cm} \\
\mathrm{n}=1 \text { (Linear) } \\
\mathrm{R}_{\mathrm{j}}=10^{-4}\end{array}$} \\
\hline
\end{tabular}

The results of this simulation are illustrated in Figure 5.11 to 5.13. Figure 5.11 shows how the shear wave is generated and propagated after the ARFI is applied in $z$ direction.

Figure 5.12, six samples of shear wave signal are illustrated at three locations inside the HIFU lesion and three locations outside of the HIFU lesion in the normal tissue.

In Figure 5.13, it has been shown how the wave energy is dissipating rapidly after it reaches to the PML region. 

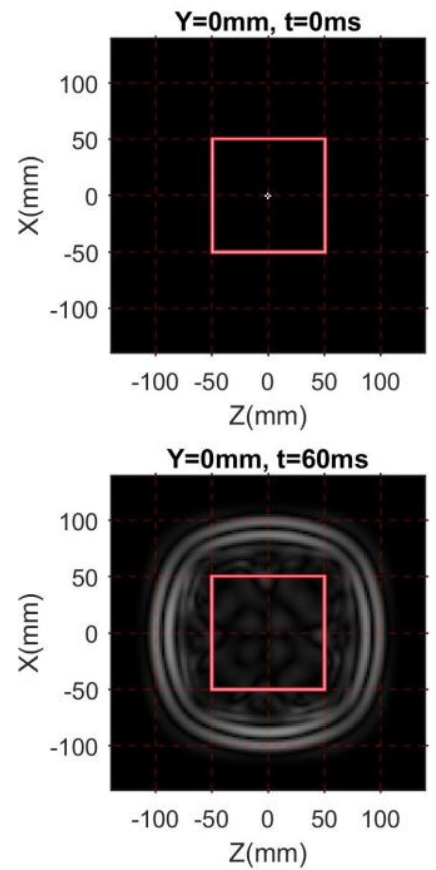
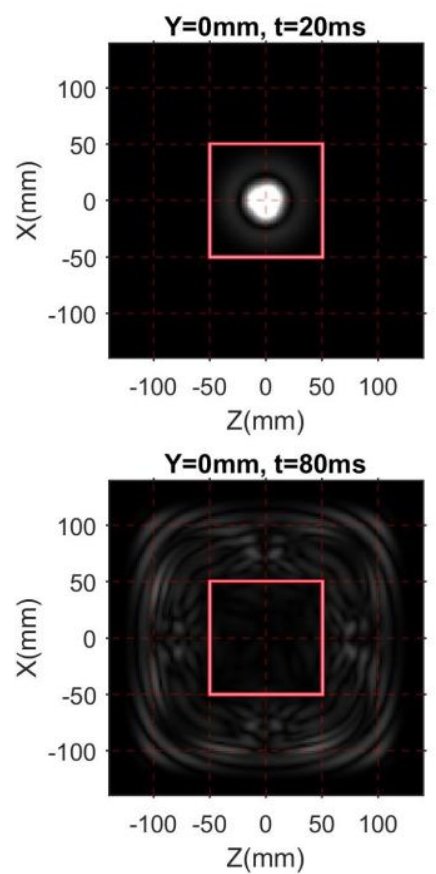
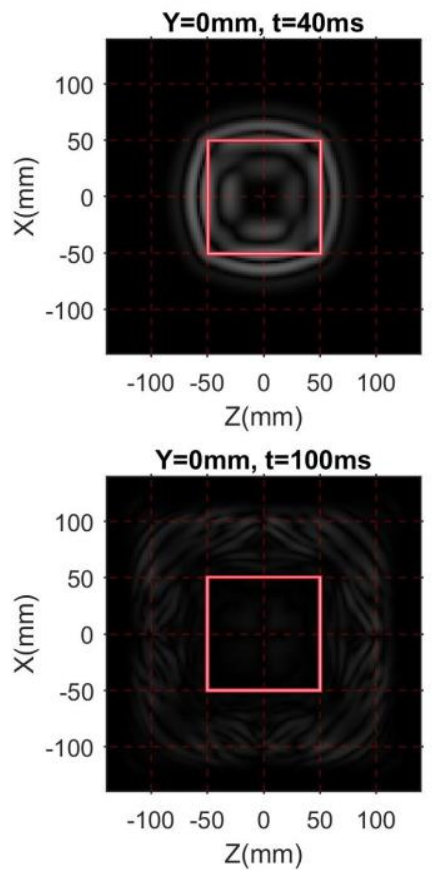

Figure 5.11. Shear wave propagation from HIFU thermal lesion to normal tissue regions. The red box denotes boundary of the induced HIFU lesion.
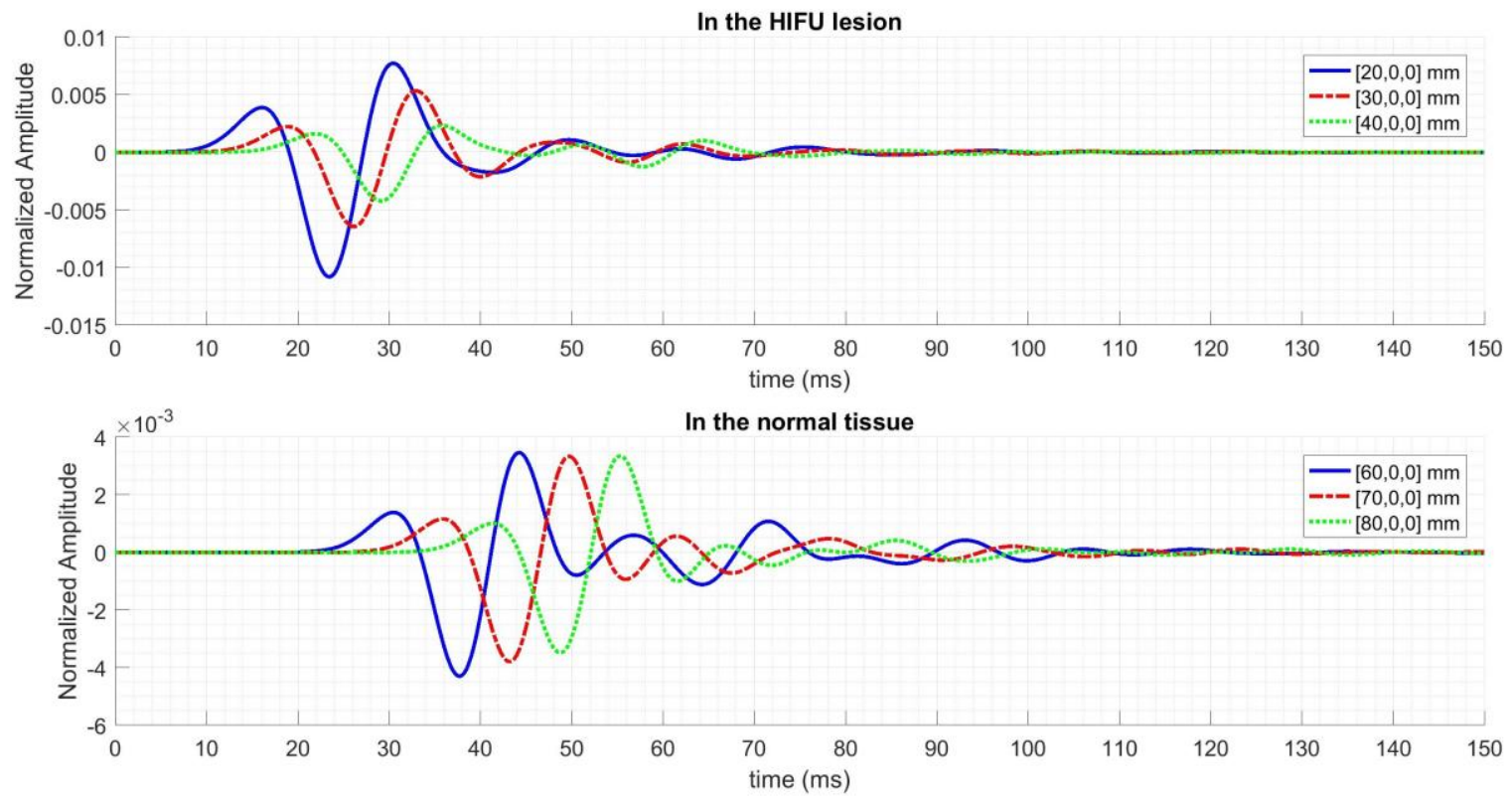

Figure 5.12. Shear wave signal at three locations inside the HIFU thermal lesion (Top) and three locations in the normal tissue (Bottom). 


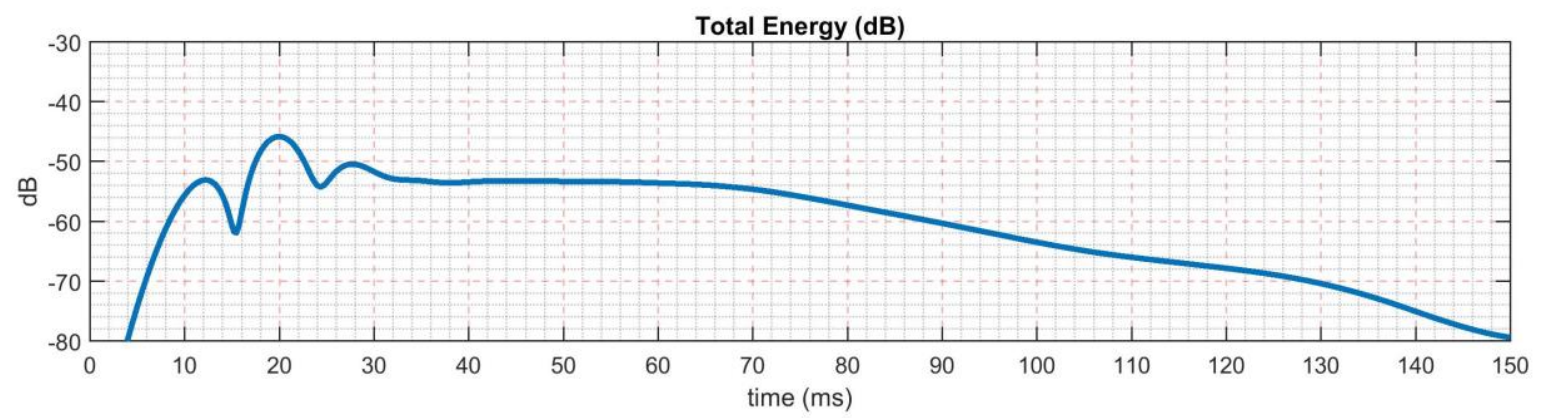

Figure 5.13. Total energy attenuation in time due to PML.

\subsubsection{Ultrasound Shear-Wave Simulation in Elliptic HIFU Lesion}

In this example, the propagation of an acoustic wave is simulated which is created within a tissuelike medium with a HIFU thermal lesion induced in the center of the normal tissue volume. In this simulation, the thermal HIFU lesion is defined in an elliptical shape which makes it alike the real HIFU lesions. The Lame's first and second coefficients and the density values used for the normal chicken breast and the thermally induced HIFU lesion are from the double indentation measurements in Table 5.3. The bulk and shear viscosities of soft tissue are usually in the range of 1-10 Pa.s [169], and the bulk viscosity is normally larger than shear viscosity; therefore, the bulk and shear viscosities are set as 10 Pa.s and 1 Pa.s, respectively. A summary of simulation parameters is provided in Table 5.5.

As illustrated in Figure 5.14, an ellipsoid-shape HIFU lesion is defined to be at the center of a volume of normal tissue and to have principle axes of $\left(r_{x}, r_{y}, r_{z}\right)$. An ARFI with a duration of $50 \mu \mathrm{s}$ and located in the center of the HIFU lesion is used as the source of shear wave. This technique is frequently used in elastography imaging and consists of a focused ultrasound beam that is absorbed in a localized region causing the generation of low frequency shear waves. 


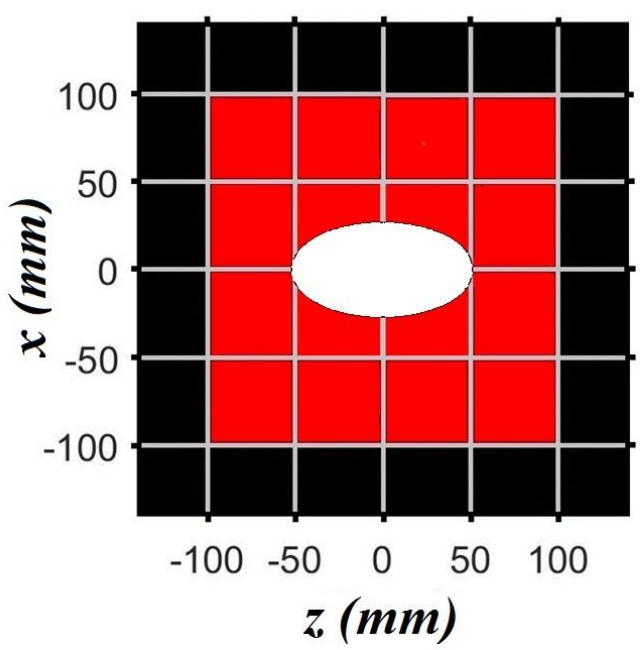

Figure 5.14. The simulation geometry: thermal HIFU lesion is inside the normal tissue, and the black area is the PML region

Figure 5.15 shows how the shear wave is generated and propagated after the ARFI is applied in $z$ direction. Six samples of shear wave signal are illustrated in Figure 5.16 at three locations inside the HIFU lesion and three locations outside of the HIFU lesion in the normal tissue. In Figure 5.17, it has been shown how the wave energy is dissipating rapidly after it reaches to the PML region.

Table 5.5: Parameters used for induced HIFU thermal lesion in chicken breast tissue

\begin{tabular}{|c|c|c|c|}
\hline & & Normal Tissue & $\begin{array}{c}\text { Elliptical HIFU } \\
\text { lesion }\end{array}$ \\
\hline $\begin{array}{l}\text { Mechanical } \\
\text { Properties (derived } \\
\text { from measurements) }\end{array}$ & $\begin{array}{l}\text { Density } \\
\text { Lame's } 1^{\text {st }} \text { parameter } \\
\text { Lame's } 2^{\text {nd }} \text { parameter } \\
\text { Bulk Viscosity } \\
\text { Shear Viscosity }\end{array}$ & $\begin{array}{c}\rho=1,004 \mathrm{~kg} / \mathrm{m}^{3} \\
\lambda=2.58 \times 10^{9} \mathrm{~Pa} \\
\mu=3.27 \times 10^{3} \mathrm{~Pa} \\
v_{p}=10 \text { Pa.s } \\
v_{s}=1 \text { Pa.s }\end{array}$ & $\begin{array}{c}\rho=1,104 \mathrm{~kg} / \mathrm{m}^{3} \\
\lambda=2.72 \times 10^{9} \mathrm{~Pa} \\
\mu=11.74 \times 10^{3} \mathrm{~Pa} \\
v_{p}=10 \text { Pa.s } \\
v_{s}=1 \text { Pa.s }\end{array}$ \\
\hline Geometry & $\begin{aligned} d x & =2 \mathrm{~mm} \\
d y & =2 \mathrm{~mm} \\
d z & =2 \mathrm{~mm}\end{aligned}$ & $\begin{array}{l}-14 \mathrm{~cm} \leq x \leq 14 \mathrm{~cm} \\
-14 \mathrm{~cm} \leq y \leq 14 \mathrm{~cm} \\
-14 \mathrm{~cm} \leq z \leq 14 \mathrm{~cm}\end{array}$ & $\begin{aligned} r_{x} & =2.5 \mathrm{~cm} \\
r_{y} & =2.5 \mathrm{~cm} \\
r_{z} & =5.0 \mathrm{~cm}\end{aligned}$ \\
\hline Simulation Duration & $d t=0.25 \mathrm{~ms}$ & \multicolumn{2}{|c|}{$0 \leq t \leq 150 \mathrm{~ms}$} \\
\hline PML Parameters & $\begin{array}{l}\text { PML Length } \\
\text { PML Order } \\
\text { Reflection Coefficient }\end{array}$ & \multicolumn{2}{|c|}{$\begin{array}{c}d=4 \mathrm{~cm} \\
n=1 \text { (Linear) } \\
R_{j}=10^{-4}\end{array}$} \\
\hline
\end{tabular}



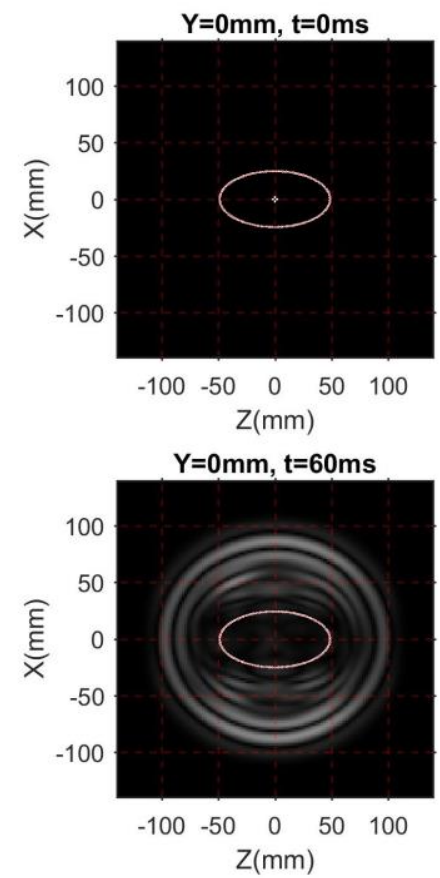
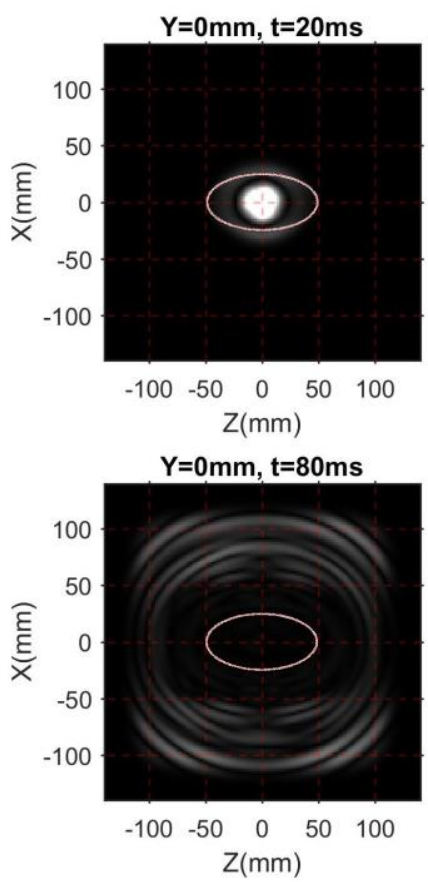
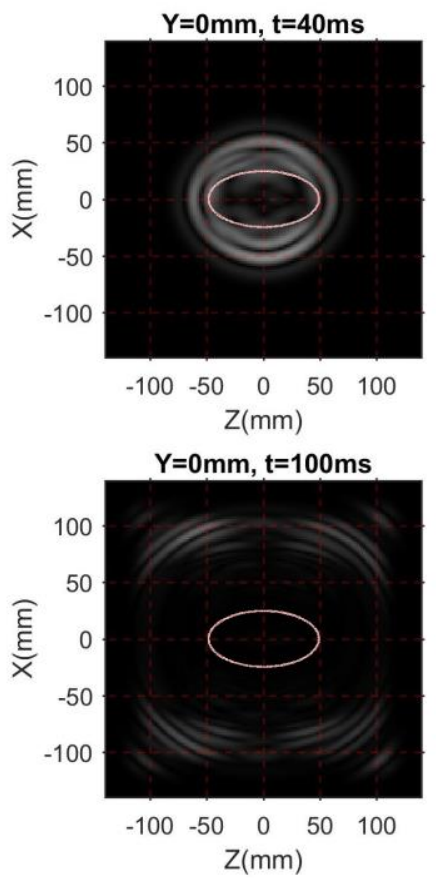

Figure 5.15. The simulation geometry: thermal HIFU lesion is inside the normal tissue, and the black area is the PML region. The outline of the elliptical HIFU region has been inserted into each frame.
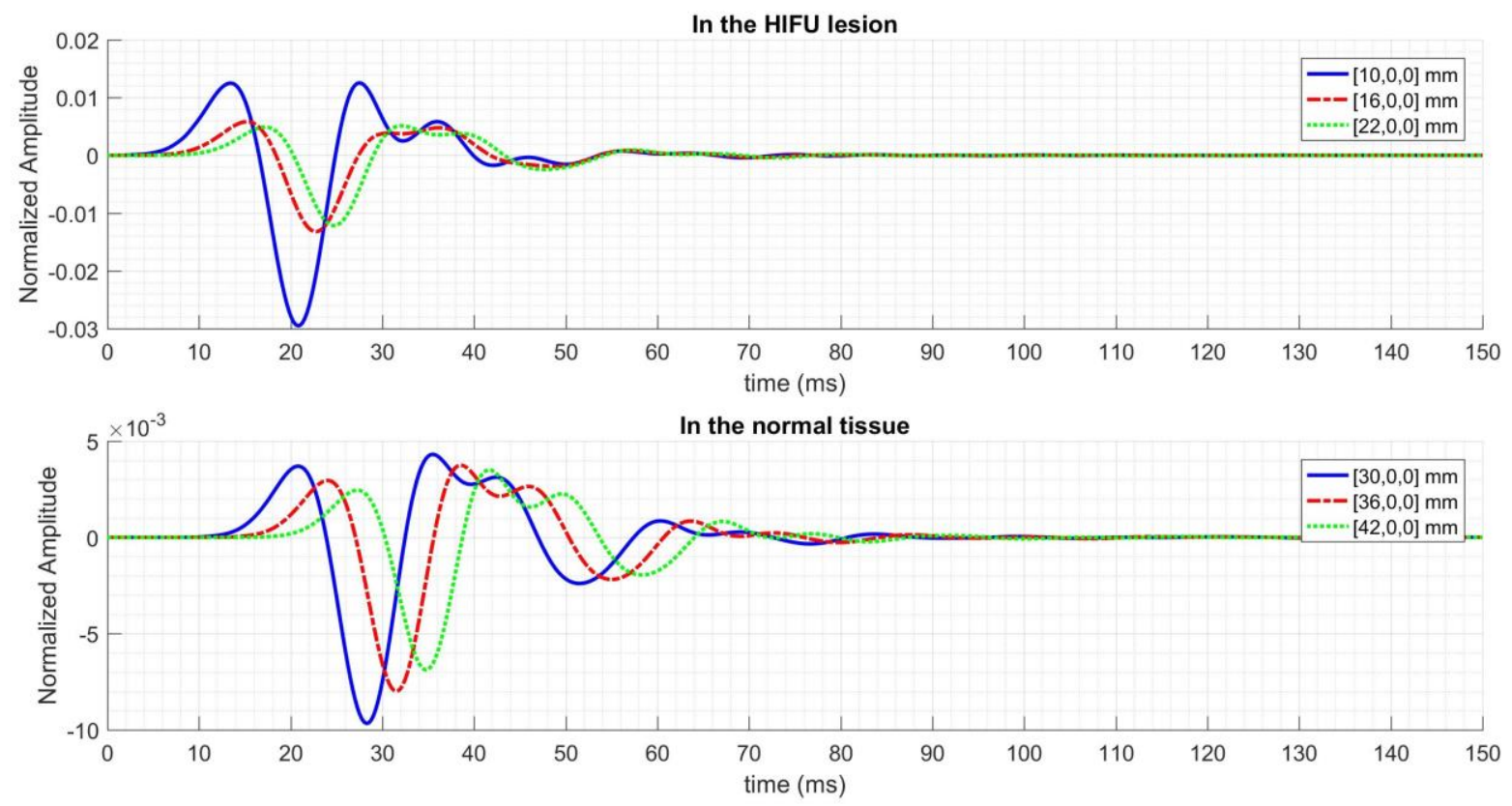

Figure 5.16. Shear wave signal at three locations inside the HIFU thermal lesion (Top) and three locations in the normal tissue (Bottom). 


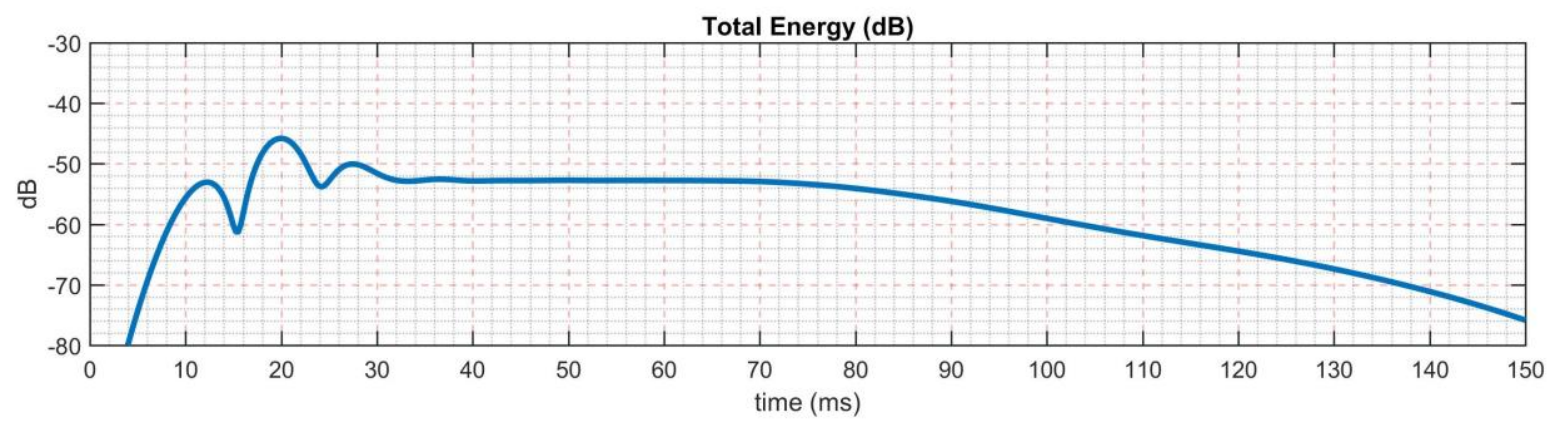

Figure 5.17. Total energy attenuation in time due to PML.

\subsection{Shear Elasticity and Viscosity Estimation}

In this section, the results of the proposed time-frequency analysis method based on the WVD techniques are studied. Since the cubic HIFU simulation was performed only because of its simplicity and as an initial study, the time-frequency method is applied to the acoustic wave simulation results of the elliptical HIFU lesion. The goal is to distinguish between the HIFU thermal lesion and the normal surrounding tissue by analyzing the shear acoustic wave propagation and estimation of tissue's viscoelastic parameters such as shear elasticity and shear viscosity. The acoustic wave propagation profile used in this section is based on the simulations in section 5.2.3. The proposed method has been applied to selected spatial points of the simulation. The results from some of the points are shown in this section.

As explained in sections 5.2.3, the HIFU simulation geometry was defined as: $-140 \mathrm{~mm} \leq x \leq 140 \mathrm{~mm}$, $-140 \mathrm{~mm} \leq y \leq 140 \mathrm{~mm},-140 \mathrm{~mm} \leq z \leq 140 \mathrm{~mm}$, with the spatial resolution of $2 \mathrm{~mm}$. However, in the time-frequency analysis, only a portion of the geometry is used for simplicity: $-40 \mathrm{~mm} \leq x \leq 40 \mathrm{~mm}$, $-40 \mathrm{~mm} \leq y \leq 40 \mathrm{~mm},-68 \mathrm{~mm} \leq z \leq 68 \mathrm{~mm}$, with the spatial steps of $2 \mathrm{~mm}$. 
In total, 12 sample points from the elliptical HIFU lesion simulation are shown in the following sections.

\subsubsection{Shear Elasticity and Viscosity Estimation in Elliptical HIFU Lesion}

The acoustic signals at four lateral points of the elliptical HIFU lesion simulation are illustrated in Figure 5.18 (top), their time-frequency profiles resulted from the WVD (middle), and the results of applying morphological operators (bottom).

The ASF method is used starting with a closing operator and a circular shape segment element with minimum size of 1 pixel and maximum size of 10 pixels. As shown in this figure, the result of applying the ASF over the time-frequency profile is to fill the gaps within the image. The red circles shown in Figure 5.18 illustrate the detected peak in the time-frequency plane which is captured by the CHT technique. This will be explained in more details later. Figure 5.19 illustrate the same results for 4 axial sample points; and Figure 5.20 show the results obtained for 4 radial sample points.

As shown in these figures, the shape, the amplitude and the frequency content of the acoustic signal changes when it is propagating in the tissue. A summary of the time of arrival, frequency and the amplitude of the acoustic wave at these 12 spatial points are shown in Table 5.6.

It should be noted that the time-of-arrival in Table 5.6 is based on the arrival of the peak of distributed energy of the signal. The value of frequency in this table represents the canter frequency of the signal that locally carries the maximum energy in the time-frequency plane. 
Table 5.6. Acoustic wave parameter captured from time-frequency analysis of 12 sample spatial points

\begin{tabular}{|c|c|c|c|c|}
\hline & $\begin{array}{c}\text { Location } \\
(\boldsymbol{x}, \boldsymbol{y}, \boldsymbol{z}) \text { in } \mathbf{~ m m}\end{array}$ & $\begin{array}{c}\text { Time of Arrival } \\
\text { in } \mathbf{~ m s}\end{array}$ & $\begin{array}{c}\text { Center Frequency } \\
\text { in Hz }\end{array}$ & Normalized Amplitude \\
\hline \multirow{4}{*}{ Lateral Points } & $(10,0,0)$ & 20.75 & 54.74 & 0.0295 \\
\cline { 2 - 5 } & $(20,0,0)$ & 24.25 & 46.92 & 0.0121 \\
\cline { 2 - 5 } & $(30,0,0)$ & 28.25 & 46.92 & 0.0097 \\
\cline { 2 - 5 } & $(40,0,0)$ & 33.75 & 46.92 & 0.0072 \\
\hline \multirow{4}{*}{ Axial Points } & $(0,0,10)$ & 25.25 & 66.47 & 0.0014 \\
\cline { 2 - 5 } & $(0,0,20)$ & 29.25 & 66.47 & 0.0016 \\
\cline { 2 - 5 } & $(0,0,30)$ & 32.75 & 84.07 & 0.0009 \\
\cline { 2 - 5 } & $(0,0,40)$ & 39.50 & 56.70 & 0.0003 \\
\hline \multirow{4}{*}{ Radial Points } & $(10,0,10)$ & 21.75 & 48.88 & 0.0131 \\
\cline { 2 - 5 } & $(20,0,20)$ & 26.25 & 52.79 & 0.0071 \\
\cline { 2 - 5 } & $(30,0,30)$ & 32.50 & 62.56 & 0.0045 \\
\cline { 2 - 5 } & $(40,0,40)$ & 40.50 & 64.52 & 0.0042 \\
\hline
\end{tabular}
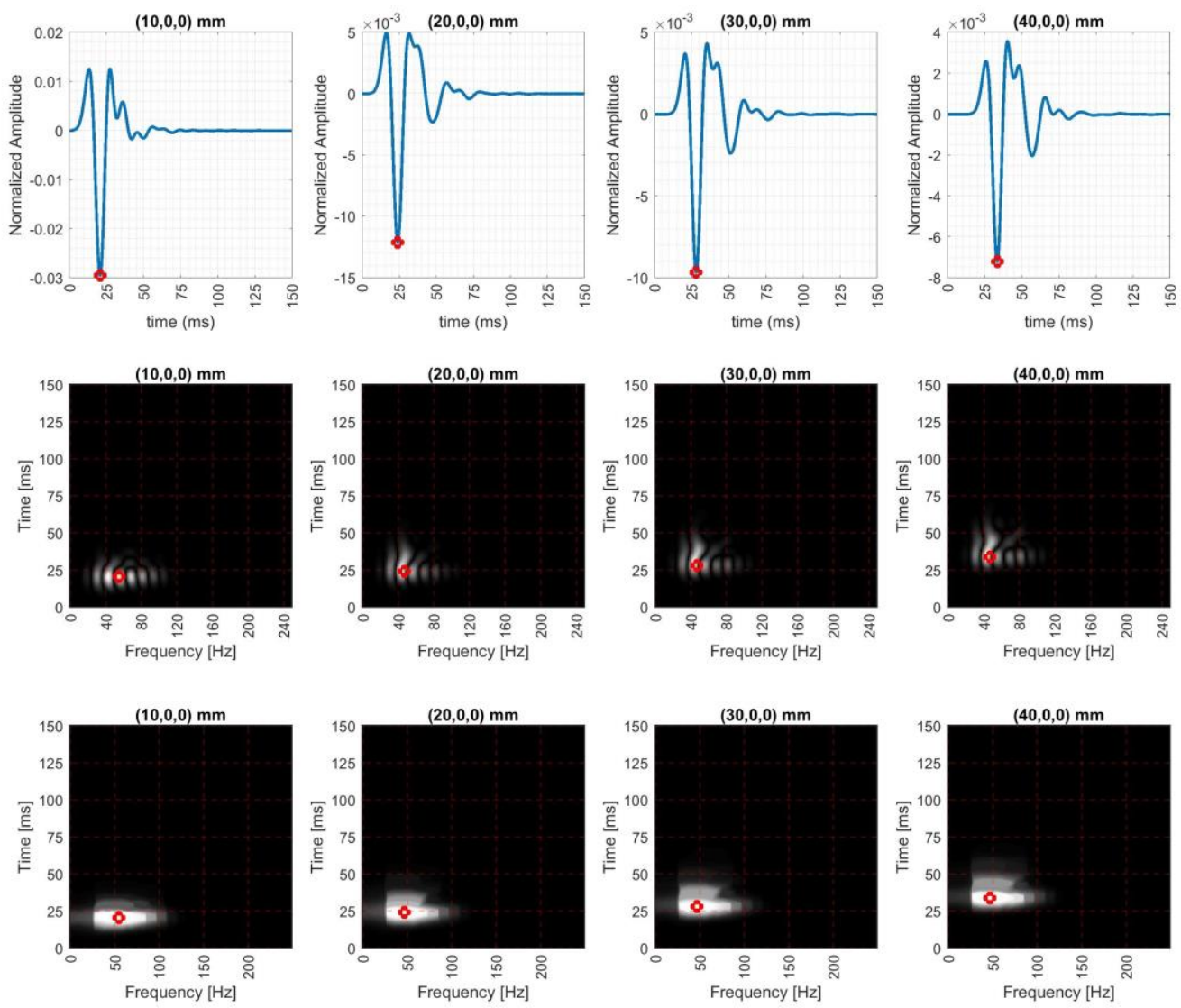

Figure 5.18. Lateral sample points in elliptical HIFU simulation, (top) acoustic signals, (middle) the WVD of the signals. (bottom) captured TF after CHT analvsis. 

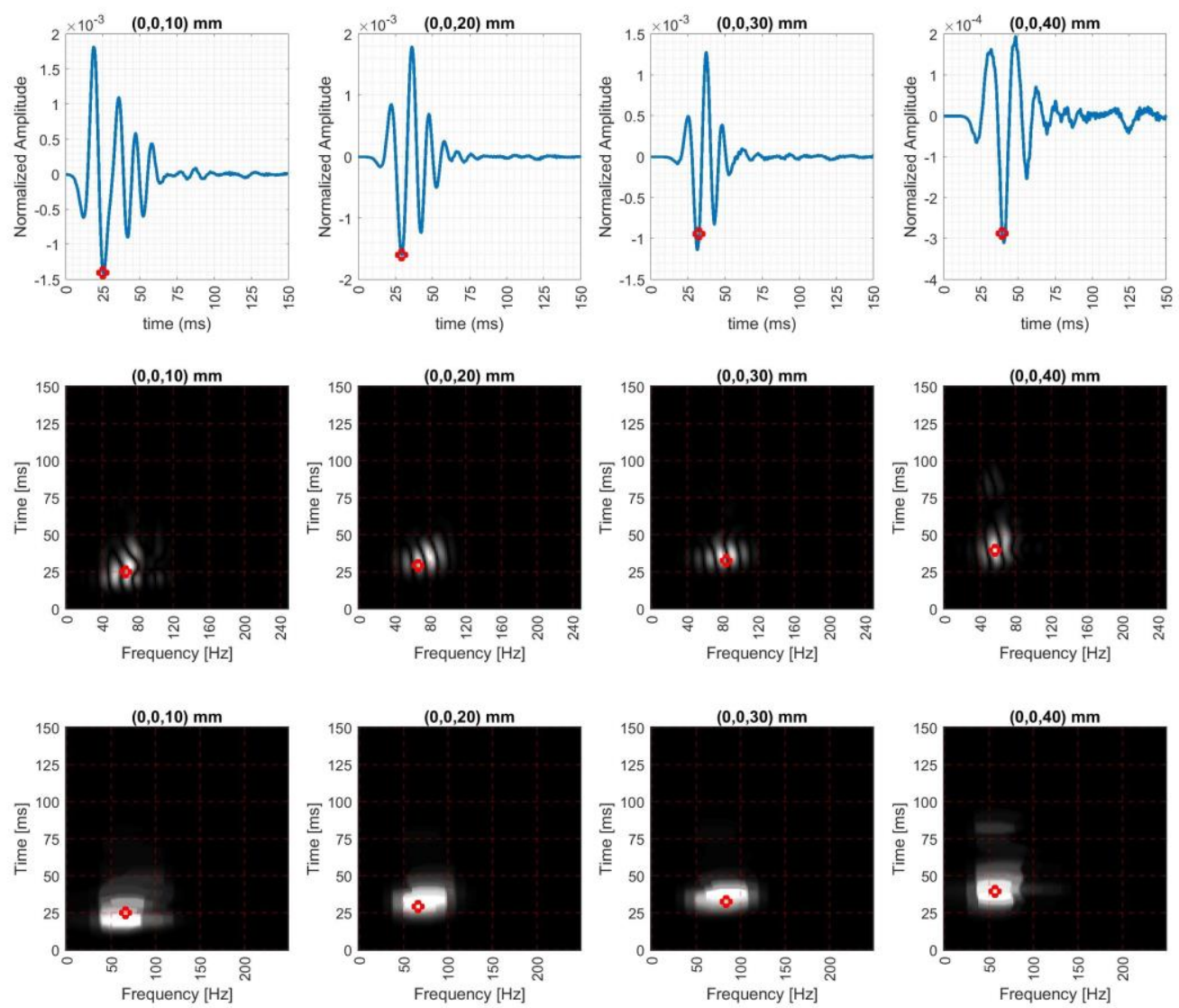

Figure 5.19. Axial sample points in elliptical HIFU simulation, (top) acoustic signals, (middle) the WVD of the signals, (bottom) captured TF after CHT analysis.

The estimated time of arrival for the points within $-40 \mathrm{~mm} \leq x \leq 40 \mathrm{~mm},-40 \mathrm{~mm} \leq y \leq 40 \mathrm{~mm}$, $-68 \mathrm{~mm} \leq z \leq 68 \mathrm{~mm}$ are shown in Figure 5.21. The speed of sound was then estimated by calculating the gradient of the 3D time-of-arrival matrix in $x, y$, and $z$ directions [4], [170]:

$$
\nabla T_{a}=\frac{\partial T_{a}}{\partial x} \hat{x}+\frac{\partial T_{a}}{\partial y} \hat{y}+\frac{\partial T_{a}}{\partial z} \hat{z}
$$

where $T_{a}$ is the 3D time-of-arrival matrix, and $\frac{\partial T_{a}}{\partial x}, \frac{\partial T_{a}}{\partial y}$ and $\frac{\partial T_{a}}{\partial z}$ are the derivatives of $T_{a}$ in $x, y$ and $z$ directions, respectively. 

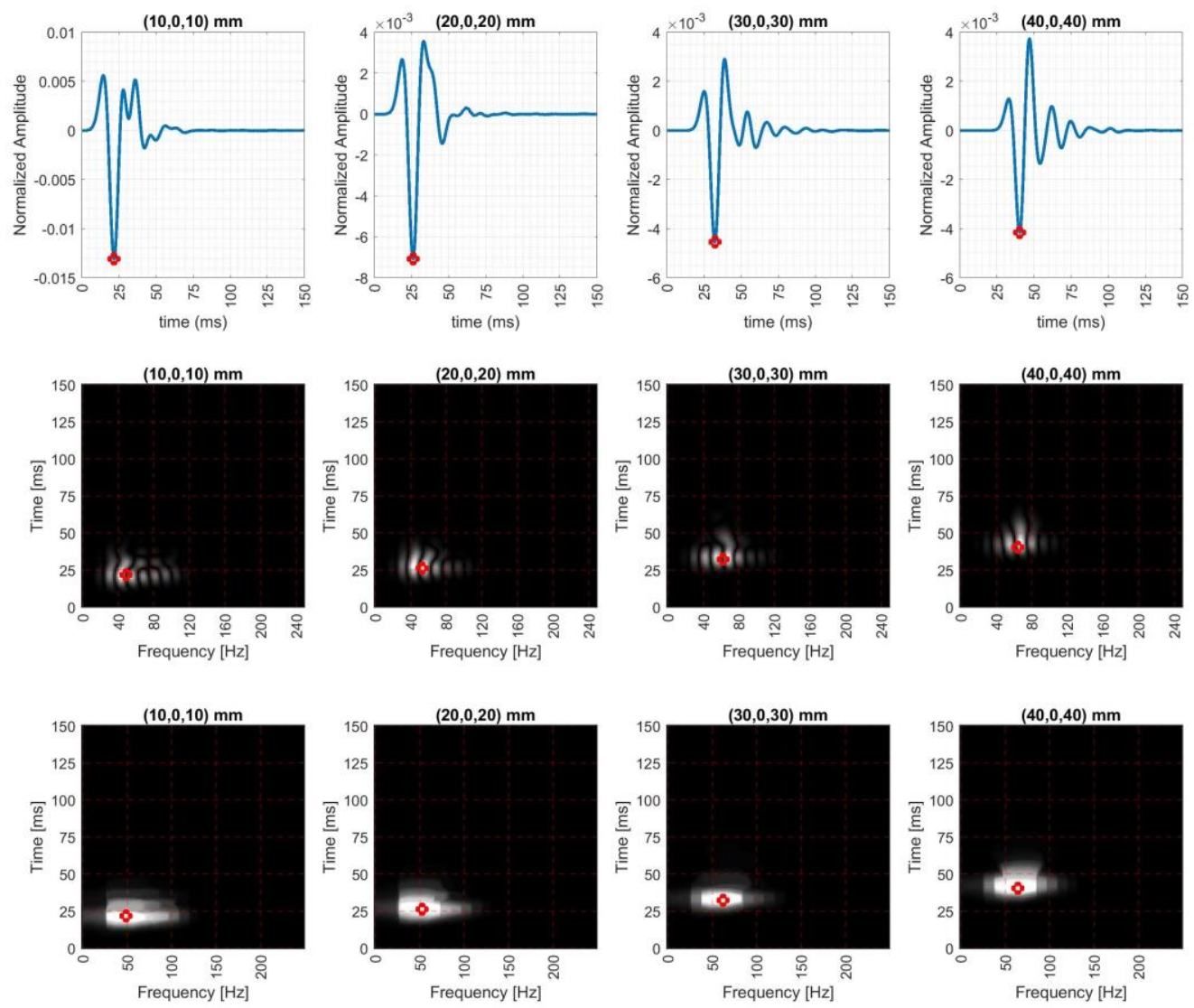

Figure 5.20. Radial sample points in elliptical HIFU simulation, (top) acoustic signals, (middle) the WVD of the signals, (bottom) captured TF after CHT analysis.

The speed of shear wave is calculated by:

$$
\begin{gathered}
c_{x}=\frac{\partial x}{\partial t}=\frac{1}{\left(\frac{\partial T a}{\partial x}\right)}, \quad c_{y}=\frac{\partial y}{\partial t}=\frac{1}{\left(\frac{\partial T a}{\partial y}\right)}, \quad c_{z}=\frac{\partial z}{\partial t}=\frac{1}{\left(\frac{\partial T_{a}}{\partial z}\right)}, \\
c=\sqrt{c_{x}^{2}+c_{y}^{2}+c_{z}^{2}}
\end{gathered}
$$

where, the components of wave propagation speed have been calculated over the whole geometry by finding the inverse of each element in $\frac{\partial T_{a}}{\partial x}, \frac{\partial T_{a}}{\partial y}$ and $\frac{\partial T_{a}}{\partial z}$ matrices. 


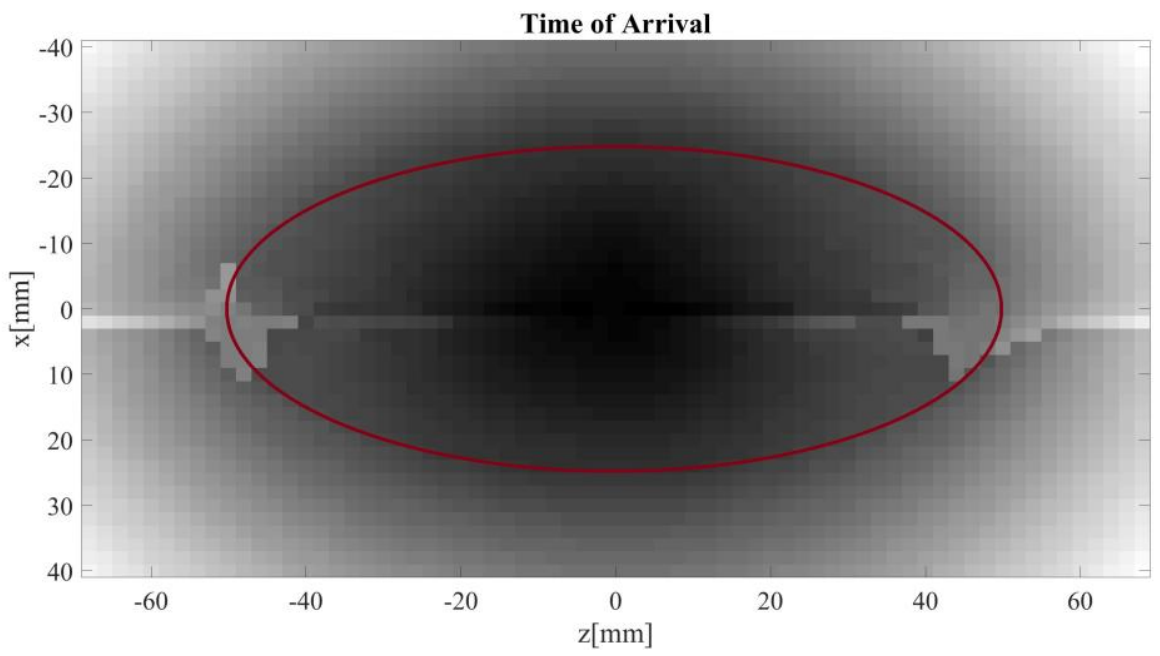

Figure 5.21. Time of arrival of the shear wave in elliptical HIFU simulation.

The speed of shear wave in $x$ and $z$ directions and the total speed of shear wave propagation are illustrated in Figure 5.22. As shown in this figure, the wave has relatively a faster propagation (brighter in the figures) in the HIFU region than the normal tissue. Figure 5.23 shows the image resulted from estimation of the shear attenuation of the media, as well as the estimated shear viscosity and shear elasticity. The latter two terms are calculated based on Eqs. (4-11) and (4-12). 


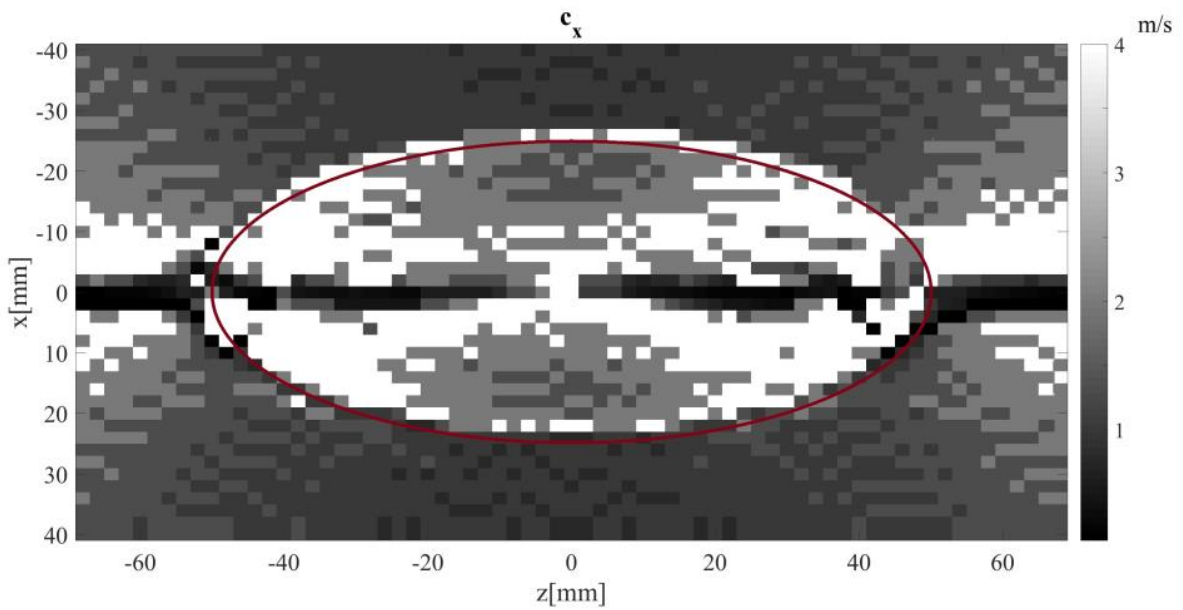

(a)

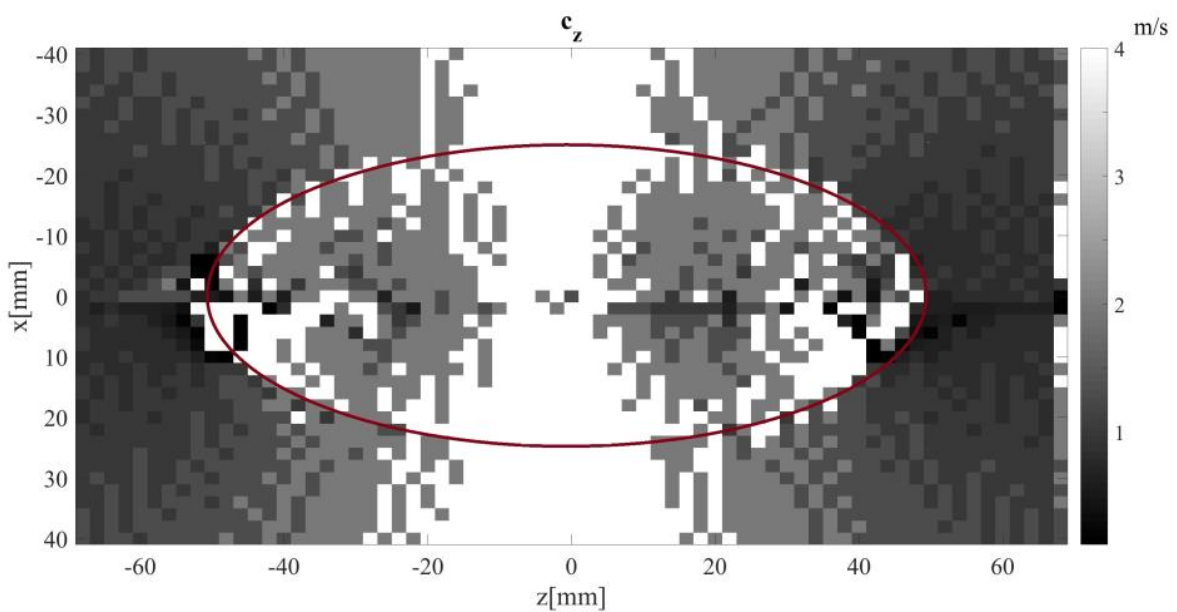

(b)

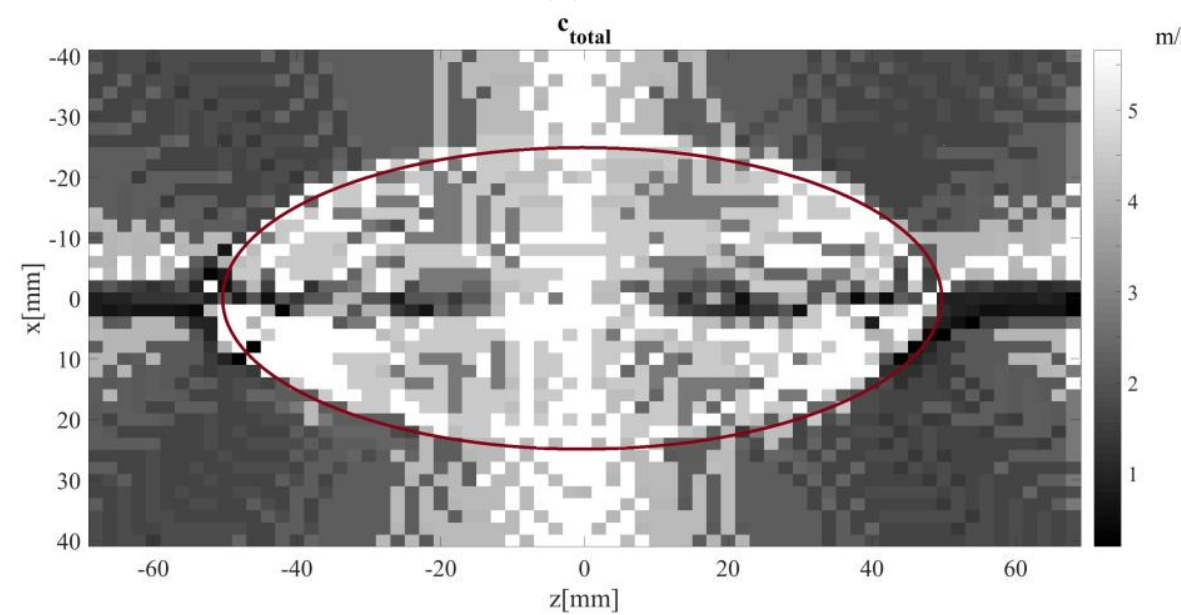

(c)

Figure 5.22. (a) speed of shear wave in $x$ direction, (b) speed of shear wave in $z$ direction, and (c) the total speed of shear wave propagating in the elliptical HIFU simulation. 


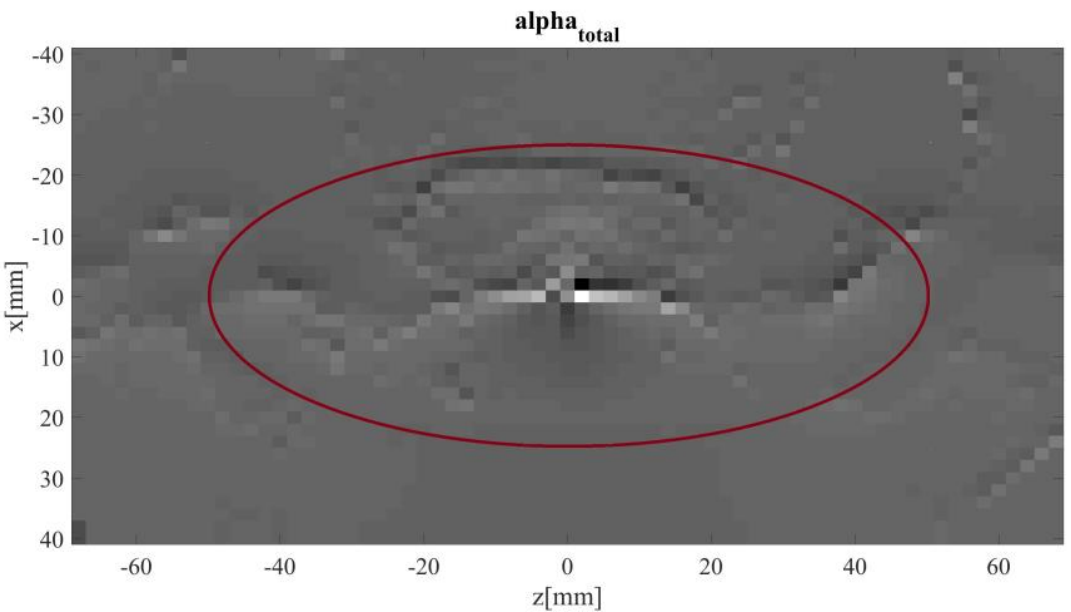

(a)

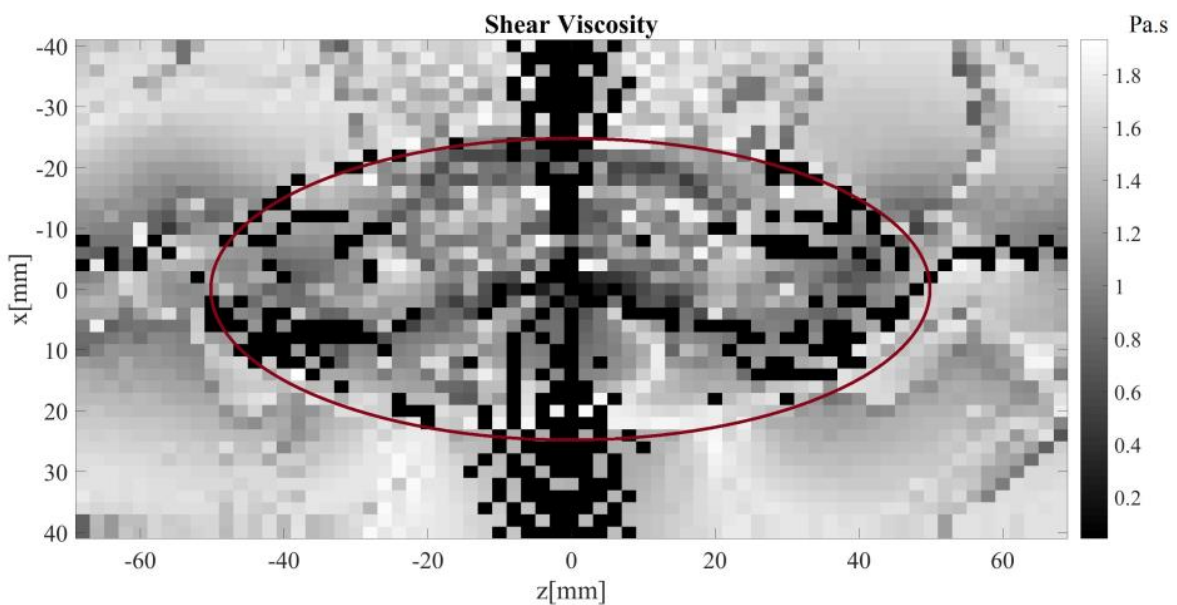

(b)

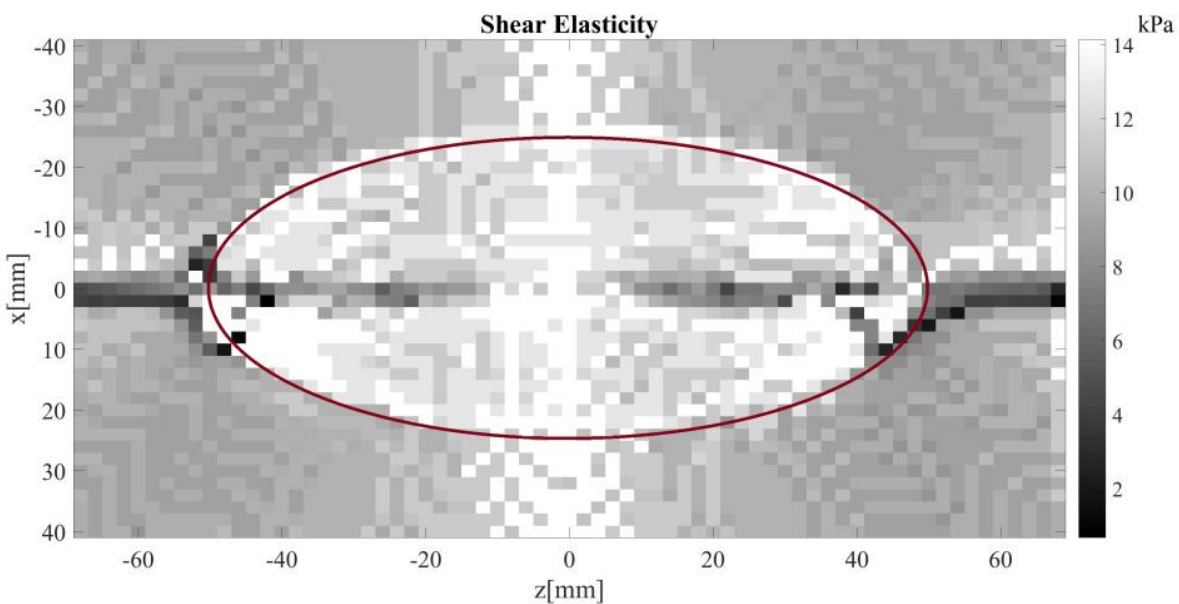

(c)

Figure 5.23. (a) estimated attenuation, (b) estimated shear viscosity, and (c) estimated shear elasticity of the elliptical HIFU simulation. 


\subsection{Discussions}

In Section 5.1.1, the proposed simulation framework has been validated for a homogeneous isotropic viscous medium. The acoustic source was defined as an impulse source and the results were compared with the analytical solution proposed by Bercoff et al. Figures 5.2 and 5.3 show that the results from the proposed finite difference method provide a good match with the Green's function solution of Bercoff et al. [56]. It should be noted that the longitudinal waves propagate much faster than shear waves in a viscous tissue-like medium. Also, an impulse source gives rise to a wide spectrum of shear waves. Moreover, the attenuation of shear waves in a viscous medium is relatively high in the ultrasound frequency range and diminishes rapidly as the frequency approaches the audio range [4]. As a result, it is only the shear wave of lower frequency spectral components that have significant amplitudes. On the other hand, the longitudinal wave created by the impulse extends over a comparatively short interval. These characteristics are seen in Figures 5.2 and 5.3 for both the simulated and Green's function predicted waveforms. As shown in these figures there is good agreement between the simulated shear wave and the results obtained using the solution given by Bercoff et al. Moreover, the longitudinal wave for both solutions consist of a very short duration oscillatory spikes. The short time oscillations following the longitudinal wave are caused by the chosen finite value of $d t$. These oscillations could be avoided with a smaller value of $d t$; but this could significantly increase the computational load.

Figures 5.4 and 5.5 compare the simulated results with and without the PML. These figures show that many numerical artifacts arise from wave reflections at the boundaries when the PML is absent. In Figure 5.4 (b) and Figure 5.5 (a), the reflections of both longitudinal and shear waves are illustrated in the simulation without the PML. The shear wave reflection can be observed in these figures because the local points are closer to the boundaries. Since these reflections affect 
the amplitude of shear wave, it can be observed that the shear wave amplitude is lower in the simulation without PML in comparison with the simulation with the PML.

As can be seen in Figure 5.6, absorption of the wave in the PML results in a significant reduction in the time variation of the total energy as computed over the entire geometry.

In Section 5.1.2, a comparison of the obtained results with those reported by Dudouit [157] for a two-layer media is presented. It has been shown in Figure 5.7 that for both simulations the upper region containing the source contains no shear wave, in agreement with the assumption that the source consisted of a displacement in the radial direction. The perfect alignment between the particle displacement and wave propagation direction fails when the wave passes through the boundary between the layers. This is shown at $t=150 \mathrm{~ms}$, when the longitudinal wave reaches the boundary causing wave-mode conversion such that shear waves are generated at the interface as the refracted and reflected waves. In general, the comparison of two sets of images shows satisfactory qualitative agreement between both methods. The major discrepancy between the two results is the differences in the brightness of the propagating acoustic wave which could be due different image normalization techniques.

In Section 5.2, the results from two numerical simulations were shown to simulate the propagation of an acoustic shear wave generated by an ARFI in soft tissue. The purpose of the simulations in this section was to show that HIFU-induced thermal lesions can be detected and imaged by using the shear wave elastography technique. In these numerical studies, the simulated tissues include a region of HIFU induced thermal lesion surrounded by normal tissue. In Section 5.2.1, a cubic HIFU lesion, as a simplified lesion geometry, is modeled and in Section 5.2.2, an elliptical HIFU thermal lesion is simulated. 
The Lame's parameters as well as the density of the normal tissue and HIFU lesion are based on experimental measurements. Young's modulus, shear modulus, and Poisson's ratio were measured using an stablished double indentation method; then, the Lame's parameters were calculated using these parameters.

In both numerical experiments, the proposed PML formulation demonstrates the effectiveness of this technique in removing the signal reflections from the boundaries.

In Section 5.3, the results of viscoelastic parameter estimation are explained. In this section, the proposed time-frequency algorithm is applied to the results from the elliptical HIFU lesion imaging simulation.

As a summary, the following steps were applied on the acoustic displacement profile in the proposed time-frequency method:

- calculation of WVD and removing the cross-terms by multiplying the time-frequency profile by the Fourier transform of the signal,

- applying ASF to remove the small gaps within the time-frequency profile,

- $\quad$ using CHT and peak detection algorithm to find the maximum energy in the time-frequency plane,

- estimation of shear wave propagation speed based on the estimated time-for-arrival,

- estimation of shear attenuation coefficient based on the changes in the amplitude of the detected waves,

- estimation of shear viscosity and elasticity using inverse Voigt model knowing speed, attenuation, and frequency of the wave.

As illustrated in Figure 5.18 to 5.23, the WVD along with the CHT and ASF methods can estimate the peak of the signals in the time-frequency plane. This results in estimation of time-of-arrival of 
the propagating shear wave within the soft tissue. It also estimates the frequency of the shear wave at every spatial point as well as the amplitude of the wave.

The shear wave propagation speed is then calculated by measuring the time it takes for the wave to propagate between two neighbor locations based on Eqs. (5-11) and (5-12). The speed of the shear wave has been calculated in different directions; then, the total speed is estimated. As shown in Figure 5.22 (a) and (b), the estimations are more accurate off-axis. The resulted calculations show an average of $3.2 \mathrm{~m} / \mathrm{s}$ and $1.85 \mathrm{~m} / \mathrm{s}$ as shear wave speeds in the HIFU lesion and the normal tissue, respectively. These values match with the expected values from the mechanical properties of the simulated media.

The results of estimating the attenuation coefficient and shear viscosity and elasticity are shown in Figure 5.23. This figure illustrates that the attenuation coefficient estimation does not distinguish the HIFU lesion from the normal tissue properly. However, the resulted shear elasticity estimation from inverse Voigt model is an improvement in HIFU detection when compared with the shear speed results.

In Figure 5.21, some artifacts present close to the boundaries of HIFU thermal lesion and the normal tissue. These artifacts are generated due to reflection and refraction of the acoustic wave in the boundaries. As the result of wave reflection and refraction, the proposed time-frequency method cannot estimate the time-of-arrival of the wave at the boundaries correctly.

There are also some artifacts in Figure 5.22 when the speed of wave propagation is calculated. These artifacts are mainly close to the axes, and they are mainly resulted from the numerical implementation of derivative. In Figure 5.22 (a), an image of $c_{x}$ is presented. In this image, the points close to $z$-axis are brighter because the wave propagation direction close to this axis is mainly in $z$ direction; however, the speed is calculated in $x$ direction. A similar result can be 
observed Figure 5.22 (a), the image of $c_{z}$, for the points close to $x$-axis. These artifacts are reduced in Figure 5.22 (c), where the total speed is calculated. As illustrated in Figure 5.23 (c) the artifacts are even more reduced after calculating the shear elasticity. For further reduction of the artifacts, appropriate image filtering techniques such as mean filter or median filter could be applied.

One of the main strengths of the proposed method is its independency in capturing the arrival time of the wave at every point. Unlike the conventional correlation-based elastography methods, the proposed time-frequency technique can estimate the wave arrival at every point independently.

On the other hand, one of the major limitations of the proposed analytical method is when it deals with waveforms with two or more frequencies close to each other. In this case, the filtering method is not effective to remove the cross-terms. In order to solve this limitation, other time-frequency methods which do not produce cross-terms such as wavelet transform might be useful.

It should be noted that HIFU thermal lesions are normally not visible in the standard B-mode imaging modality [119]; therefore, Figures (5.22) and (5.23) illustrate significant achievements, obtained from the proposed time-frequency algorithm, by the detecting the HIFU thermal lesion and distinguishing it from the normal surrounding tissue.

The limitations of the proposed methods are discussed in more details in the Future Work section. 


\section{Chapter 6}

\section{Conclusions and Future Work}

\subsection{Conclusions}

As the first main goal of this thesis, a simulation framework capable of modeling acoustic full wave propagation in 3D geometries was proposed and implemented. The framework can simulate acoustic wave propagation in elastic or viscoelastic heterogeneous media with any arbitrary acoustic wave source. In this framework, a 3D PML formulation was included in the elastodynamic wave equation to simulate the propagation of acoustic waves in 3D media with nonreflecting and absorbing boundaries. For the numerical implementation, the finite difference formulation was used to discretize the elastodynamic wave equation and the numerical stability analysis was studied.

Then, the implementation of the proposed framework and the PML formulation was validated by comparing its results in homogeneous viscous isotropic media with the results from a Green's function formulation. The Green's functions were obtained from the well-established published research works that presented closed-form equations for infinite viscoelastic homogeneous isotropic media. The proposed simulation framework has also been validated for a multilayer inhomogeneous isotropic simulation using seismological parameters. 
After validation of the numerical simulation framework, two simulation examples of acoustic wave propagation in the field of biomedical ultrasound were presented in which thermally-induced HIFU lesions placed in the middle of normal soft tissue to demonstrate the capabilities of this simulation framework in biomedical elastography applications. The parameters used in these simulations were based on double-indentation mechanical measurements of the viscoelastic parameters of ex vivo chicken breast samples with and without high intensity focused ultrasound induced thermal lesions.

The other main goal of this thesis was to present an elastography detection method based on a time-frequency analysis of acoustic wave propagation in soft tissue. The goal was to differentiate the HIFU thermal lesion from the normal tissue. The WVD algorithm is used along with other image processing methods such as ASF and CHT techniques to estimate the time-of-arrival, the center frequency of the wave carrying the maximum energy, and the amplitude of the propagating acoustic shear wave in a simulated HIFU thermal lesion located within normal soft tissue. Then, the shear elasticity and viscosity of the media was estimated by inverse Voigt model and the HIFU lesion was differentiated from the normal tissue.

The main contributions of this thesis research work are summarized as:

- To the best of author's knowledge, this is the first time that a 3D method has been proposed to simulate the generalized elastodynamic full wave equation for viscoelastic heterogeneous media that includes a PML technique.

- The proposed numerical framework can simulate full wave propagation for elastography purposes.

- More details are obtained by analyzing acoustic wave propagation for elastography purposes using time-frequency WVD along with the image processing techniques. The 
proposed method not only calculates the time-of-arrival of the wave, it also estimates the central frequency of the wave and the attenuation coefficient, and generate 3D maps of the speed of wave propagation, shear elasticity and shear viscosity of the medium.

- In the proposed method, the estimation of time-of-arrival of the wave can be performed independently at each spatial point.

\subsection{Future Work}

Since the acoustics wave simulation is a beneficial tool for many research fields, any improvement or added feature to the simulation framework is an added value to this work.

The proposed framework in this research was mainly based on the elastodynamic wave equation which is a linear equation and could handle an arbitrary acoustic source geometry. Therefore, the following future works are proposed:

1- In this study, the ARFI that was used as the acoustic source in the HIFU lesion simulation was based on a single-point source within the soft tissue. One possible study is to substitute the point source with a spatially distributed acoustic source obtained from the profile of a HIFU acoustic field at its focal region. The initial study could be based on a linear profile of the HIFU field; then, this could be extended to a nonlinear distributed source. The nonlinear profile of the transducer can be calculated by nonlinear wave equations such as KZK or Westervelt equations and used to calculate a distributed acoustic force.

2- In the current study, fixed increments in time and space discretization were used. Another possible research could be to use variable increments to improve the performance and the speed of simulation. This could potentially help to simulate different sources of 
longitudinal and shear waves with significantly different speeds and frequencies in a single simulation framework.

3- Another possible future work is to validate the proposed simulation framework in anisotropic cases, such as acoustic wave propagation in bone. The elastodynamic wave equations used in the proposed method are also valid for the anisotropic media; however, the simulation needs to be validated for anisotropic studies.

4- The proposed simulation can also be studied in more depths by experimental measurements of the acoustic field generated from various types of source. This might be achieved either by hydrophone measurements, or using research-based ultrasound scanners such as Verasonics scanner.

There are also possible future works in the proposed time-frequency analysis of the acoustic wave:

1- Other methods of generating shear wave can be studied by the proposed time-frequency algorithms, such as transient or vibrational shear waves.

2- The proposed method can be experimentally studied on shear acoustic wave generated from ARFI within soft tissue.

3- The diagnostic capabilities of the proposed method can be studied to differentiate cancerous or abnormal tissue from normal tissue. As mentioned previously, shear elasticity has a wider range of values than bulk modulus in soft tissue; therefore, the proposed method could potentially be used to differentiate normal and abnormal tissues.

4- The proposed time-frequency method can potentially be used on commercial ultrasound scanners through FPGA or GPU implementations.

There are other potential future projects that could be performed to continue this research study. The above suggestions are just a few of them. 


\section{References:}

[1] K. Aki and P. G. Richards, Quantitative Seismology, 2nd ed. Univ Science Books, 2002.

[2] P. M. Shearer, Introduction to Siesmology, 2nd ed., no. 1. Cambridge University Press, 2009.

[3] G. Harvey, A. Gachagan, and T. Mutasa, "Review of high-power ultrasound-industrial applications and measurement methods," IEEE Trans. Ultrason. Ferroelectr. Freq. Control, vol. 61, no. 3, pp. 481-495, 2014.

[4] R. S. C. Cobbold, "Foundations of Biomedical Ultrasound," Oxford Univ. Press, pp. 45$51,2007$.

[5] G. ter Haar, "Therapeutic applications of ultrasound," Prog. Biophys. Mol. Biol., vol. 93, no. 1-3, pp. 111-129, 2007.

[6] H. T. Lutz and H. A. Gharbi, Manual of Diagnostic Ultrasound in Infectious Tropical Diseases. Springer, 2006.

[7] G. ter Haar, "Interventional Oncology," in Interventional Oncology, P. R. Mueller and A. Adam, Eds. 2012, pp. 51-63.

[8] G. ter Haar, "Review Therapeutic ultrasound," Eur. J. Ultrasound, vol. 9, no. 1999, pp. 3$9,1999$.

[9] H. G. Hosseini, A. Alizad, and M. Fatemi, "Integration of vibro-acoustography imaging modality with the traditional mammography," Int. J. Biomed. Imaging, vol. 2007, pp. 1-8, 
2007.

[10] W. D. O'Brien Jr, “Assessing the risks for modern diagnostic ultrasound imaging," Jpn. J. Appl. Phys., vol. 37, pp. 2781-2788, 1998.

[11] Y. Li and J. a. Zagzebski, "Computer model for harmonic ultrasound imaging," IEEE Trans. Ultrason. Ferroelectr. Freq. Control, vol. 47, no. 5, pp. 1259-1272, 2000.

[12] P. L. M. J. van Neer et al., "Comparison of fundamental, second harmonic, and superharmonic imaging: A simulation study,” J. Acoust. Soc. Am., vol. 130, no. 5, p. 3148, 2011.

[13] T. Szabo, Diagnostic ultrasound imaging: inside out. 2004.

[14] P. N. Burns, D. Hope Simpson, and M. A. Averkiou, "Nonlinear imaging," Ultrasound Med. Biol., vol. 26, no. SUPPL. 1, pp. 19-22, 2000.

[15] J. Ophir, I. Céspedes, H. Ponnekanti, Y. Yazdi, and X. Li, "Elastography: a quantitative method for imaging the elasticity of biological tissues.," Ultrason. Imaging, vol. 13, pp. $111-134,1991$.

[16] P. Asbach et al., "Assessment of liver viscoelasticity using multifrequency MR elastography," Magn. Reson. Med., vol. 60, no. 2, pp. 373-379, 2008.

[17] S. J. Kirkpatrick, R. K. Wang, and D. D. Duncan, "OCT-based elastography for large and small deformations.," Opt. Express, vol. 14, no. 24, pp. 11585-11597, 2006.

[18] A. S. Khalil, R. C. Chan, A. H. Chau, B. E. Bouma, and M. R. K. Mofrad, "Tissue elasticity estimation with optical coherence elastography: Toward mechanical characterization of in vivo soft tissue," Ann. Biomed. Eng., vol. 33, no. 11, pp. 1631-1639, 2005.

[19] J. Bercoff, M. Tanter, and M. Fink, "Supersonic Shear Imaging : A New Technique," IEEE Trans. Ultrason. Ferroelectr. Freq. Control, vol. 51, no. 4, pp. 396-409, 2004. 
[20] M. M. Doyley, "Model-based elastography: a survey of approaches to the inverse elasticity problem," Phys. Med. Biol., vol. 57, no. 3, pp. R35-R73, 2012.

[21] M. Tanter and M. Fink, "Ultrafast imaging in biomedical ultrasound," IEEE Trans. Ultrason. Ferroelectr. Freq. Control, vol. 61, no. 1, pp. 102-119, 2014.

[22] K. Darge, B. Zieger, W. Rohrschneider, S. Ghods, R. Wunsch, and J. Troeger, "Contrastenhanced harmonic imaging for the diagnosis of vesicoureteral reflux in pediatric patients," AJR Am J Roentgenol, vol. 177, no. 6, pp. 1411-1415, 2001.

[23] C. C. Coussios and R. a. Roy, "Applications of Acoustics and Cavitation to Noninvasive Therapy and Drug Delivery," Annu. Rev. Fluid Mech., vol. 40, no. 1, pp. 395-420, 2008.

[24] P. M. Eberwein and J. D. Denstedt, "Treatment of Renal Calculi with Extracorporeal Shock Wave Lithotripsy," Can. Fam. Physician, vol. 38, pp. 1670-1675, 1992.

[25] M. R. Bailey et al., "Cavitation detection during shock-wave lithotripsy," Ultrasound Med. Biol., vol. 31, no. 9, pp. 1245-1256, 2005.

[26] W. W. Roberts, T. L. Hall, K. Ives, J. S. Wolf, J. B. Fowlkes, and C. A. Cain, "Pulsed cavitational ultrasound: A noninvasive technology for controlled tissue ablation (histotripsy) in the rabbit kidney," J. Urol., vol. 175, no. 2, pp. 734-738, 2006.

[27] S. Petropavlovsky and S. Tsynkov, "Non-deteriorating time domain numerical algorithms for Maxwell's electrodynamics,” J. Comput. Phys., vol. 336, pp. 1-35, 2017.

[28] G. Ter Haar, "Ultrasound focal beam surgery," Ultrasound Med. Biol., vol. 21, no. 9, pp. 1089-1100, 1995.

[29] D. Das, M. Gupta, H. Kaur, and A. Kalucha, "Elastography: The Next Step,” J. Quant. Anal. Sport., vol. 7, no. 4, pp. 9-12, 2011.

[30] K. J. Parker, M. M. Doyley, and D. J. Rubens, "Corrigendum: Imaging the elastic properties 
of tissue: the 20 year perspective," Phys. Med. Biol., vol. 57, no. 16, pp. 5359-5360, 2012.

[31] I. Céspedes, J. Ophir, H. Ponnekanti, and N. Maklad, "Elastography: elasticity imaging using ultrasound with application to muscle and breast in vivo.," Ultrasonic imaging, vol. 15, no. 2.pp. 73-88, 1993.

[32] J. Belaïd, N., Cespedes, I., Thijssen, J.M., Ophir, "Lesion Detection in Simulated Elastographic and Echographic Images: A Psychophysical Study," Ultrasound Med. Biol., vol. 20, no. 9, pp. 877-891, 1994.

[33] M. M. Doyley, S. Srinivasan, E. Dimidenko, N. Soni, and J. Ophir, "Enhancing the performance of model-based elastography by incorporating additional a priori information in the modulus image reconstruction process.," Phys. Med. Biol., vol. 51, no. 1, pp. 95-112, 2006.

[34] H. Ponnekanti, J. Ophir, and I. Cespedes, “Axial stress distributions between coaxial compressors in elastography: an analytical model," Ultrasound Med. Biol., vol. 18, no. 5, pp. 667-673, 1992.

[35] J. Ophir, R. K. Miller, H. Ponnekanti, I. Cespedes, and A. D. Whittaker, "Elastography of Beef Muscle,” Meat Sci., vol. 36, pp. 239-250, 1994.

[36] H. Ponnekanti, J. Ophir, and I. Cespedes, "Ultrasonic imaging of the stress distribution in elastic media due to an external compressor," Ultrasound in Med. \& Biol., vol. 20, no. 1. pp. 27-33, 1994.

[37] H. Ponnekanti, J. Ophir, Y. Huang, and I. Céspedes, "Fundamental mechanical limitations on the visualization of elasticity contrast in elastography.," Ultrasound Med. Biol., vol. 21, no. 4, pp. 533-543, 1995.

[38] F. G. Mitri et al., "Vibro-acoustography imaging of permanent prostate brachytherapy seeds 
in an excised human prostate - Preliminary results and technical feasibility," Ultrasonics, vol. 49, no. 3, pp. 389-394, 2009.

[39] F. G. Mitri, B. J. Davis, J. F. Greenleaf, and M. Fatemi, "In vitro comparative study of vibro-acoustography versus pulse-echo ultrasound in imaging permanent prostate brachytherapy seeds," Ultrasonics, vol. 49, no. 1, pp. 31-38, 2009.

[40] A. Alizad, L. E. Wold, J. F. Greenleaf, and M. Fatemi, "Imaging mass lesions by vibroacoustography: Modeling and experiments," IEEE Trans. Med. Imaging, vol. 23, no. 9, pp. 1087-1093, 2004.

[41] C. Pislaru et al., "In vivo vibroacoustography of large peripheral arteries.," Invest. Radiol., vol. 43, no. 4, pp. 243-52, 2008.

[42] A. Alizad, M. Walch, J. F. Greenleaf, and M. Fatemi, "Vibrational Characteristics of Bone Fracture and Fracture Repair: Application to Excised Rat Femur," J. Biomech. Eng., vol. 128, no. 3, p. 300, 2006.

[43] K. R. Nightingale, R. W. Nightingale, M. L. Palmeri, and G. E. Trahey, “A finite element model of remote palpation of breast lesions using radiation force: factors affecting tissue displacement.," Ultrason. Imaging, vol. 22, no. 1, pp. 35-54, 2000.

[44] K. Nightingale, M. Palmeri, and G. Trahey, "Analysis of contrast in images generated with transient acoustic radiation force," Ultrasound Med. Biol., vol. 32, no. 1, pp. 61-72, 2006.

[45] B. J. Fahey, K. R. Nightingale, R. C. Nelson, M. L. Palmeri, and G. E. Trahey, “Acoustic radiation force impulse imaging of the abdomen: Demonstration of feasibility and utility," Ultrasound Med. Biol., vol. 31, no. 9, pp. 1185-1198, 2005.

[46] B. J. Fahey, S. J. Hsu, P. D. Wolf, R. C. Nelson, and G. E. Trahey, "Liver ablation guidance with acoustic radiation force impulse imaging," Phys. Med. Biol., vol. 51, no. 15, pp. 3785- 
3808, 2006.

[47] B. J. Fahey, R. C. Nelson, D. P. Bradway, S. J. Hsu, D. M. Dumont, and G. E. Trahey, "In vivo visualization of abdominal malignancies with acoustic radiation force elastography," Phys. Med. Biol., vol. 53, no. 1, pp. 279-293, 2008.

[48] S. H. Cho, J. Y. Lee, J. K. Han, and B. I. Choi, "Acoustic Radiation Force Impulse Elastography for the Evaluation of Focal Solid Hepatic Lesions: Preliminary Findings," Ultrasound Med. Biol., vol. 36, no. 2, pp. 202-208, 2010.

[49] L. Zhai et al., "Acoustic Radiation Force Impulse Imaging of Human Prostates: Initial In Vivo Demonstration," Ultrasound Med. Biol., vol. 38, no. 1, pp. 50-61, 2012.

[50] S. A. Eyerly, S. J. Hsu, S. H. Agashe, G. E. Trahey, Y. Li, and P. D. Wolf, “An in vitro assessment of acoustic radiation force impulse imaging for visualizing cardiac radiofrequency ablation lesions," J. Cardiovasc. Electrophysiol., vol. 21, no. 5, pp. 557$563,2010$.

[51] A. P. Tierney, D. M. Dumont, A. Callanan, G. E. Trahey, and T. M. McGloughlin, "Acoustic radiation force impulse imaging on ex vivo abdominal aortic aneurysm model.," Ultrasound Med. Biol., vol. 36, no. 5, pp. 821-832, 2010.

[52] R. H. Behler, T. C. Nichols, H. Zhu, E. P. Merricks, and C. M. Gallippi, “ARFI Imaging for Noninvasive Material Characterization of Atherosclerosis Part II: Toward In Vivo Characterization," Ultrasound Med. Biol., vol. 35, no. 2, pp. 278-295, 2009.

[53] A. Thitaikumar, T. a Krouskop, B. S. Garra, and J. Ophir, "Visualization of bonding at an inclusion boundary using axial-shear strain elastography: a feasibility study.," Phys. Med. Biol., vol. 52, no. 9, pp. 2615-2633, 2007.

[54] J. McLaughlin and D. Renzi, "Shear wave speed recovery in transient elastography and 
supersonic imaging using propagating fronts," Inverse Probl., vol. 22, no. 2, pp. 681-706, 2006.

[55] A. Giannoula and R. S. C. Cobbold, "Propagation of shear waves generated by a modulated finite amplitude radiation force in a viscoelastic medium," IEEE Trans. Ultrason. Ferroelectr. Freq. Control, vol. 56, no. 3, pp. 575-588, 2009.

[56] J. Bercoff, M. Tanter, M. Muller, and M. Fink, "The role of viscosity in the impulse diffraction field of elastic waves induced by the acoustic radiation force," IEEE Trans. Ultrason. Ferroelectr. Freq. Control, vol. 51, no. 11, pp. 1523-1536, 2004.

[57] G. P. Berry, J. C. Bamber, N. R. Miller, P. E. Barbone, N. L. Bush, and C. G. Armstrong, "Towards an acoustic model-based poroelastic imaging method: II. experimental investigation," Ultrasound Med. Biol., vol. 32, no. 12, pp. 1869-1885, 2006.

[58] P. R. Perriñez, F. E. Kennedy, E. E. W. Van Houten, J. B. Weaver, and K. D. Paulsen, "Magnetic Resonance Poroelastography: An Algorithm for Estimating the Mechanical Properties of Fluid-Saturated Soft Tissues,” vol. 29, no. 3, pp. 746-755, 2010.

[59] P. R. Perriñez, F. E. Kennedy, E. E. W. Van Houten, J. B. Weaver, and K. D. Paulsen, "Modeling of soft poroelastic tissue in time-harmonic MR elastography," IEEE Trans. Biomed. Eng., vol. 56, no. 3, pp. 598-608, 2009.

[60] M. S. Richards, P. E. Barbone, and A. a Oberai, "Quantitative three-dimensional elasticity imaging from quasi-static deformation: a phantom study.," Phys. Med. Biol., vol. 54, no. 3, pp. 757-779, 2009.

[61] F. Kallel and M. Bertrand, "Tissue elasticity reconstruction using linear perturbation method," IEEE Trans. Med. Imaging, vol. 15, no. 3, pp. 299-313, 1996.

[62] M. M. Doyley, S. Srinivasan, S. A. Pendergrass, Z. Wu, and J. Ophir, "Comparative 
evaluation of strain-based and model-based modulus elastography," Ultrasound Med. Biol., vol. 31, no. 6, pp. 787-802, 2005.

[63] U. Zaleska-Dorobisz, K. Kaczorowski, A. Pawlu??, A. Puchalska, and M. Inglot, "Ultrasound elastography - review of techniques and its clinical applications.," Adv. Clin. Exp. Med., vol. 23, no. 4, pp. 645-655, 2014.

[64] Y. Yamakoshi, T. Nakajima, T. Kasahara, M. Yamazaki, R. Koda, and N. Sunaguchi, "Shear wave imaging of breast tissue by color doppler shear wave elastography," IEEE Trans. Ultrason. Ferroelectr. Freq. Control, vol. 64, no. 2, pp. 340-348, 2017.

[65] A. Itoh et al., "Breast disease: clinical application of US elastography for diagnosis.," Radiology, vol. 239, no. 2, pp. 341-350, 2006.

[66] H. Zhi et al., "Semi-quantitating Stiffness of Breast Solid Lesions in Ultrasonic Elastography,” Acad. Radiol., vol. 15, no. 11, pp. 1347-1353, 2008.

[67] T. R. Kumm and M. M. Szabunio, "Elastography for the Characterization of Breast Lesions :," Cancer Control, vol. 17, no. 3, pp. 156-161, 2010.

[68] A. Athanasiou et al., "Breast lesions: quantitative elastography with supersonic shear imaging--preliminary results.," Radiology, vol. 256, no. 1, pp. 297-303, 2010.

[69] M. Tanter et al., "Quantitative Assessment of Breast Lesion Viscoelasticity: Initial Clinical Results Using Supersonic Shear Imaging," Ultrasound Med. Biol., vol. 34, no. 9, pp. 13731386, 2008.

[70] J. Bamber et al., "EFSUMB guidelines and recommendations on the clinical use of ultrasound elastography. Part 1: Basic principles and technology.," Ultraschall der Medizin, vol. 34, no. 2, pp. 169-84, 2013.

[71] D. Cosgrove et al., "EFSUMB Guidelines and Recommendations on the Clinical Use of 
Ultrasound Elastography. Part 2: Clinical Applications," Ultraschall Med, vol. 34, pp. 238253, 2013.

[72] G. D. Kirk et al., "Assessment of Liver Fibrosis by Transient Elastography in Persons with Hepatitis C Virus Infection or HIV-Hepatitis C Virus Coinfection,” vol. 48, no. 7, pp. 963972, 2009.

[73] S. L. Friedman, "Evolving challenges in hepatic fibrosis.PDF," Nat. Rev. Gastroenterol. Hepatol., vol. 7, pp. 425-436, 2010.

[74] E. Bavu et al., "Noninvasive In Vivo Liver Fibrosis Evaluation Using Supersonic Shear Imaging: A Clinical Study on 113 Hepatitis C Virus Patients," Ultrasound Med. Biol., vol. 37, no. 9, pp. 1361-1373, 2011.

[75] S. Chen et al., "Assessment of liver viscoelasticity by using shear waves induced by ultrasound radiation force," Radiology, vol. 266, no. 3, pp. 964-970, 2013.

[76] M. A. Aguilo, J. C. Brigham, W. Aquino, and M. Fatem, “An Inverse Problem Approach for Elasticity Imaging through Vibroacoustics," IEEE Trans. Med. Imaging, vol. 29, no. 4, pp. 1012-1021, 2010.

[77] L. Sandrin et al., "Transient elastography: a new noninvasive method for assessment of hepatic fibrosis," Ultrasound Med. Biol., vol. 29, no. 12, pp. 1705-1713, 2003.

[78] C. Vorländer, J. Wolff, S. Saalabian, R. H. Lienenlüke, and R. A. Wahl, "Real-time ultrasound elastography-a noninvasive diagnostic procedure for evaluating dominant thyroid nodules," Langenbeck's Arch. Surg., vol. 395, no. 7, pp. 865-871, 2010.

[79] J. Y. Fung, C. LAI, and M. YUEN, "Clinical Application of Transient Liver Diseases Assessment of Fibrosis," Hongkong Med. Diary, vol. 14, no. 11, pp. 22-25, 2009.

[80] L. Castera et al., "Prospective comparison of transient elastography, Fibrotest, APRI, and 
liver biopsy for the assessment of fibrosis in chronic hepatitis C," Gastroenterology, vol. 128, no. 2, pp. 343-350, 2005.

[81] J. M. Correas, A. M. Tissier, A. Khairoune, G. Khoury, D. Eiss, and O. Hélénon, "Ultrasound elastography of the prostate: State of the art," Diagn. Interv. Imaging, vol. 94, no. 5, pp. 551-560, 2013.

[82] F. G. Mitri, P. Trompette, and J. Y. Chapelon, "Improving the Use of Vibro-Acoustography for Brachytherapy Metal Seed Imaging: A Feasibility Study,” IEEE Trans. Med. Imaging, vol. 23, no. 1, pp. 1-6, 2004.

[83] K. KONIG, U. SCHEIPERS, A. PESAVENTO, A. LORENZ, H. ERMERT, and T. SENGE, "Initial Experiences With Real-Time Elastography Guided Biopsies of the Prostate," J. Urol., vol. 174, no. 1, pp. 115-117, 2005.

[84] A. Sarvazyan, T. J. Hall, M. W. Urban, M. Fatemi, S. R. Aglyamov, and B. S. Garra, "an Overview of Elastography - an Emerging Branch of Medical Imaging," Curr. Med. Imaging Rev., vol. 7, no. 4, pp. 255-282, 2011.

[85] A. Lyshchik et al., "Thyroid gland tumor diagnosis at US elastography.," Radiology, vol. 237, no. 1, pp. 202-211, 2005.

[86] U. Bae, M. Dighe, T. Dubinsky, S. Minoshima, V. Shamdasani, and Y. Kim, "Ultrasound thyroid elastography using carotid artery pulsation: preliminary study.," J. ultrasound Med., vol. 26, no. 6, pp. 797-805, 2007.

[87] J. J. Choi et al., "Role of sonographic elastography in the differential diagnosis of axillary lymph nodes in breast cancer," J. Ultrasound Med., vol. 30, no. 4, pp. 429-436, 2011.

[88] J. Bojunga, E. Herrmann, G. Meyer, S. Weber, S. Zeuzem, and M. Friedrich-Rust, "Realtime elastography for the differentiation of benign and malignant thyroid nodules: a meta- 
analysis," Thyroid, vol. 20, no. 10, pp. 1145-1150, 2010.

[89] I. Sporea, R. Sirli, S. Bota, M. Vlad, A. Popescu, and I. Zosin, “ARFI elastography for the evaluation of diffuse thyroid gland pathology: Preliminary results," World J Radiol, vol. 4, no. 4, pp. 174-178, 2012.

[90] S. Wojcinski, J. Dupont, W. Schmidt, M. Cassel, and P. Hillemanns, "Real-time ultrasound elastography in 180 axillary lymph nodes: elasticity distribution in healthy lymph nodes and prediction of breast cancer metastases," BMC Med. Imaging, vol. 12, no. 1, p. 35, 2012.

[91] J. G. Lynn, R. L. Zwemer, A. J. Chick, and A. E. Miller, "A new method for the generation and use of focused ultrasound in experimental biology," J. Gen. Physiol., vol. 26, no. 2, pp. 179-193, 1942.

[92] K. Hynynen et al., "A clinical, noninvasive, MR imaging-monitored ultrasound surgery method.," Radiographics, vol. 16, no. 1, pp. 185-195, 1996.

[93] D. Gianfelice, A. Khiat, M. Amara, A. Belblidia, and Y. Boulanger, "MR Imaging-guided Focused US Ablation of Breast Cancer: Histopathologic Assessment of EffectivenessInitial Experience," Radiology, vol. 227, no. 3, pp. 849-855, 2003.

[94] N. T. Sanghvi et al., "Noninvasive surgery of prostate tissue by high intensity focused ultrasound: An updated report," Eur. J. Ultrasound, vol. 9, no. 1, pp. 19-29, 1999.

[95] F. Wu et al., "A randomised clinical trial of high-intensity focused ultrasound ablation for the treatment of patients with localised breast cancer.," Br. J. Cancer, vol. 89, no. 12, pp. 2227-2233, 2003.

[96] A. Kanthabalan et al., "Focal salvage high-intensity focused ultrasound in radiorecurrent prostate cancer," BJU Int., vol. 120, no. 2, pp. 246-256, 2017.

[97] A. H. Chan, V. Y. Fujimoto, D. E. Moore, R. W. Martin, and S. Vaezy, “An image-guided 
high intensity focused ultrasound device for uterine fibroids treatment," Med. Phys., vol. 29, no. 11, pp. 2611-2620, 2002.

[98] Z. G. Liang et al., "Experimental study on ablation of leiomyoma by combination highintensity focused ultrasound and iodized oil in vitro," J. Obstet. Gynaecol. Res., vol. 43, no. 10, pp. 1578-1584, 2017.

[99] V. P. Nguyen, J. Oh, S. Park, and H. Wook Kang, "Feasibility of photoacoustic evaluations on dual-thermal treatment of ex vivo bladder tumors," J. Biophotonics, vol. 10, no. 4, pp. 577-588, 2017.

[100] K. U. Köhrmann, M. S. Michel, J. Gaa, E. Marlinghaus, and P. Alken, "High intensity focused ultrasound as noninvasive therapy for multilocal renal cell carcinoma: case study and review of the literature.," J. Urol., vol. 167, no. 6, pp. 2397-2403, 2002.

[101] A. G. Visioli et al., "Preliminary results of a phase I dose escalation clinical trial using focused ultrasound in the treatment of localised tumours.," Eur. J. ultrasound, vol. 9, no. 1999, pp. 11-18, 1999.

[102] S. Vaezy et al., "Liver Hemostasis With High-Intensity Ultrasound," J Ultrasound Med, vol. 23, pp. 217-225, 2004.

[103] A. Fatemi and M. A. C. Kane, "High-intensity focused ultrasound effectively reduces waist circumference by ablating adipose tissue from the abdomen and flanks: A retrospective case series," Aesthetic Plast. Surg., vol. 34, no. 5, pp. 577-582, 2010.

[104] A. Fatemi, "High-Intensity Focused Ultrasound Effectively Reduces Adipose Tissue," Semin. Cutan. Med. Surg., vol. 28, no. 4, pp. 257-262, 2009.

[105] S. G. Fabi, "Noninvasive skin tightening: Focus on new ultrasound techniques," Clin. Cosmet. Investig. Dermatol., vol. 8, pp. 47-52, 2015. 
[106] H. Park, E. Kim, J. Kim, Y. Ro, and J. Ko, "High-intensity focused ultrasound for the treatment of wrinkles and skin laxity in seven different facial areas," Ann. Dermatol., vol. 27, no. 6, pp. 688-693, 2015.

[107] S. Vaezy et al., "REAL-TIME VISUALIZATION OF HIGH-INTENSITY FOCUSED ULTRASOUND TREATMENT USING ULTRASOUND IMAGING,” Ultrasound Med. Biol., vol. 29, no. 2, pp. 33-42, 2011.

[108] G. ter Haar, "High intensity ultrasound.," Semin. Laparosc. Surg., vol. 8, no. 1, pp. 77-89, 2001.

[109] P. Lai, J. R. McLaughlan, A. B. Draudt, T. W. Murray, R. O. Cleveland, and R. a Roy, "Real-time monitoring of high-intensity focused ultrasound lesion formation using acoustooptic sensing.," Ultrasound Med. Biol., vol. 37, no. 2, pp. 239-252, 2011.

[110] R. Illing and A. Chapman, "The clinical applications of high intensity focused ultrasound in the prostate," Int. J. Hyperth., vol. 23, no. 2, pp. 183-191, 2007.

[111] N. T. Sanghvi et al., "Clinical validation of real-time tissue change monitoring during prostate tissue ablation with high intensity focused ultrasound," J. Ther. Ultrasound, vol. 5, no. 1, p. 24, 2017.

[112] P. Rangraz, H. Behnam, P. Sobhe Bidari, N. Shakhssalim, and J. Tavakkoli, "Dynamic Changes in the Acousto-Mechanical and Statistical Parameters of Tissue During High Intensity Focused Ultrasound (HIFU) Treatment," Biomed. Eng. Appl. Basis Commun., vol. 26, pp. 1-9, Feb. 2014.

[113] P. Rangraz, H. Behnam, P. Sobhebidari, and J. Tavakkoli, "Real-Time Monitoring of HighIntensity Focused Ultrasound Thermal Therapy Using the Manifold Learning Method,” Ultrasound Med. Biol., vol. 40, no. 12, pp. 2841-2850, 2014. 
[114] R. Souchon et al., "Visualisation of HIFU lesions using elastography of the human prostate in vivo: Preliminary results," Ultrasound Med. Biol., vol. 29, no. 7, pp. 1007-1015, 2003.

[115] G. Pareek et al., "Elastographic measurements of in-vivo radiofrequency ablation lesions of the kidney.," J. Endourol., vol. 20, no. 11, pp. 959-964, 2006.

[116] T. Wu, J. P. Felmlee, J. F. Greenleaf, S. J. Riederer, and R. L. Ehman, “Assessment of thermal tissue ablation with MR elastography.," Magn. Reson. Med., vol. 45, no. 1, pp. 8087, 2001.

[117] H.-L. Liu, M.-L. Li, P.-H. Tsui, M.-S. Lin, S.-M. Huang, and J. Bai, “A unified approach to combine temperature estimation and elastography for thermal lesion determination in focused ultrasound thermal therapy.," Phys. Med. Biol., vol. 56, no. 1, pp. 169-186, 2011.

[118] A. K. Thittai, B. Galaz, and J. Ophir, "Visualization of HIFU-Induced lesion boundaries by axial-shear strain elastography: A feasibility study," Ultrasound Med. Biol., vol. 37, no. 3, pp. 426-433, 2011.

[119] R. Iwasaki et al., "Monitoring of high-intensity focused ultrasound treatment by shear wave elastography induced by two-dimensional-array therapeutic transducer," Jpn. J. Appl. Phys., vol. 55, no. 7, 2016.

[120] J. P. Berenger, "A perfectly matched layer for the absorption of electromagnetic waves," $J$. Comput. Phys., vol. 114, pp. 185-200, 1994.

[121] O. Ramadan, "Systematic split-step perfectly matched layer formulations for modelling dispersive open region finite difference time domain applications," vol. 5, no. January, pp. 1062-1066, 2011.

[122] B. Sjögreen and N. A. Petersson, "Perfectly matched layers for Maxwell's equations in second order formulation," J. Comput. Phys., vol. 209, no. 1, pp. 19-46, 2005. 
[123] K. Duru and G. Kreiss, "A well-posed and discretely stable perfectly matched layer for elastic wave equations in second order formulation," Commun. Comput. Phys., vol. 11, no. 5, pp. 1643-1672, 2012.

[124] D. P. Connolly, A. Giannopoulos, and M. C. Forde, "A higher order perfectly matched layer formulation for finite-difference time-domain seismic wave modeling," Geophysics, vol. 80, no. 1, pp. 1-16, 2015.

[125] T. Ishizuka and K. Okubo, "Formulation and examination of the perfectly matched layer for sound field analyses using the constrained interpolation profile method," Acoust. Sci. Technol., vol. 34, no. 5, pp. 378-381, 2013.

[126] R. Cimpeanu, A. Martinsson, and M. Heil, "A parameter-free perfectly matched layer formulation for the finite-element-based solution of the Helmholtz equation," J. Comput. Phys., vol. 296, pp. 329-347, 2015.

[127] H. Assi and R. S. C. Cobbold, "A second-order, perfectly matched layer formulation to model 3D transient wave propagation in anisotropic elastic media," pp. 1-21, 2016.

[128] H. Assi and R. S. C. Cobbold, "Compact second-order time-domain perfectly matched layer formulation for elastic wave propagation in two dimensions," Math. Mech. Solids, vol. 22, no. 1, pp. 20-37, 2017.

[129] K. Sagiyama, S. Govindjee, and P.-O. Persson, "An efficient time-domain perfectly matched layers formulation for elastodynamics elastodynamics on spherical domains," Int. J. Numer. Methods Eng., vol. 100, pp. 419-441, 2014.

[130] B. Arnal, M. Pernot, and M. Tanter, "Monitoring of thermal ablation therapy based on shear modulus changes: Shear wave thermometry and shear wave lesion imaging," 2010 IEEE Int. Ultrason. Symp., pp. 1522-1525, 2010. 
[131] B. Arnal, M. Pernot, and M. Tanter, "Monitoring of thermal therapy based on shear modulus changes: I. Shear wave thermometry," IEEE Trans. Ultrason. Ferroelectr. Freq. Control, vol. 58, no. 2, pp. 369-378, 2011.

[132] S. Chen et al., "Shearwave dispersion ultrasound vibrometry (SDUV) for measuring tissue elasticity and viscosity," IEEE Trans. Ultrason. Ferroelectr. Freq. Control, vol. 56, no. 1, pp. 55-62, 2009.

[133] B. Arnal, M. Pernot, and M. Tanter, "Monitoring of thermal therapy based on shear modulus changes: II. Shear wave imaging of thermal lesions," IEEE Trans. Ultrason. Ferroelectr. Freq. Control, vol. 58, no. 2, pp. 369-378, 2011.

[134] M. Sandsten, TIME-FREQUENCY ANALYSIS OF NON-STATIONARY PROCESSES: An Introduction. 2013.

[135] L. Cohen, “Time-frequency distributions-a review," Proc. IEEE, vol. 77, no. 7, pp. 941981, 1989.

[136] J. OPHIR et al., "Imagerie acoustique et optique des milieux biologiques," Appl. Phys., vol. 2, no. 4, pp. 1193-1212, 2001.

[137] A. Giannoula and R. S. C. Cobbold, "Mapping the local shear modulus and viscosity using a transient finite-amplitude modulated radiation force," Ultrasonics, vol. 51, no. 3, pp. 340$351,2011$.

[138] Y. Zheng et al., "Shear Wave Propagation in Soft Tissue and Ultrasound Vibrometry Chapter," in Wave Propagation Theories and Applications, 2013, pp. 1-24.

[139] A. Giannoula, R. S. C. Cobbold, and A. Bezerianos, "Estimating the local viscoelastic properties from dispersive shear waves using time-frequency ridge analysis," Ultrasonics, vol. 53, no. 2, pp. 534-544, 2013. 
[140] P. J. Durka, “Time-frequency analyses of EEG,” 1996.

[141] B. Boashash, Time Frequency Signal Analysis and Processing: A Comprehensive Reference, 2nd ed. 2015.

[142] S. Q. S. Qian and D. C. D. Chen, "Joint time-frequency analysis," IEEE Signal Process. Mag., vol. 16, no. 2, 1999.

[143] S. Blanco, R. Q. Quiroga, O. A. Rosso, and S. Kochen, "Time-frequency analysis of electroencephalogram series," Phys. Rev. E, vol. 51, no. 3, pp. 2624-2631, 1995.

[144] B. Boashash and G. Frazer, "Time-varying higher-order spectra, generalised WignerVilledistribution and the analysis of underwater acoustic data," [Proceedings] ICASSP-92 1992 IEEE Int. Conf. Acoust. Speech, Signal Process., vol. 5, pp. 193-196, 1992.

[145] S. Mallat, A wavelet tour of signal processing: The Sparse way, 3rd ed. 2009.

[146] D. Gabor, “Theory of Communication," J. Inst. Electr. Eng. - Part III Radio Commun. Eng., vol. 93 , no. 26 , pp. 429-457, 1946.

[147] F. Hlawatsch and F. Auger, Time-Frequency Analysis: Concepts and Methods. John Wiley \& Sons, Inc., 2008.

[148] I. Tosic and P. Frossard, "Dictionary Learning, What is the right representation for my signal?," Signal Process. Mag. IEEE, vol. 28, no. 2, pp. 27-38, 2011.

[149] P. S. Addison, The illustrated wavelet transform handbook: Introductory theory and applications in science, engineering, medicine and finance, 2nd ed. 2016.

[150] J. O’Toole, M. Mesbah, and B. Boashash, “A Discrete Time and Frequency Wigner-Ville Distribution: Properties and Implementation," in Digital Signal Processing and Communication Systems, 2005.

[151] M. Sandsten, Time-Freqyuency Analysis of Non-Stationary Processes. 2013. 
[152] J. C. S. Cardoso, M. G. Ruano, and P. J. Fish, "Nonstationarity Broadening Reduction in Pulsed Doppler Spectrum Measurements Using Time-Frequency Estimators," IEEE Trans. Biomed. Eng., vol. 43, no. 12, 1996.

[153] J. M. Girault, D. Kouame, a. Ouahabi, and F. Patat, "Micro-emboli detection: An ultrasound doppler signal processing viewpoint," IEEE Trans. Biomed. Eng., vol. 47, no. 11, pp. 1431$1439,2000$.

[154] Y. Li and X. Zheng, "Wigner-Ville distribution and its application in seismic attenuation estimation,” Appl. Geophys., vol. 4, no. 4, pp. 245-254, 2007.

[155] L. Xu and Y. Yang, "Parameter estimation of underwater moving sources by using matched Wigner transform,” Appl. Acoust., vol. 101, pp. 5-14, 2016.

[156] H. Assi, "Time-domain modeling of elastic and acoustic wave propagation in unbounded media , with application to metamaterials by Hisham Assi," University of Toronto, 2016.

[157] Y. Dudouit, "Spatio-temporal refinement using a discontinuous Galerkin approach for elastodynamic in a high performance computing framework," UNIVERSITÉ DE BORDEAUX, 2014.

[158] R. Martin and D. Komatitsch, “An unsplit convolutional perfectly matched layer technique improved at grazing incidence for the viscoelastic wave equation," Geophys. J. Int., vol. 179, no. 1, pp. 333-344, 2009.

[159] J. G. CHARNEY, R. FJÖRTOFT, and J. NEUMANN, "Numerical Integration of the Barotropic Vorticity Equation,” Tellus, vol. 2, no. 4, pp. 237-254, 1950.

[160] J. Bercoff, M. Tanter, and M. Fink, "Supersonic Shear Imaging: A New Technique for Soft Tissue Elasticity Mapping," IEEE Trans. Ultrason. Ferroelectr. Freq. Control, vol. 51, no. 4, pp. 396-409, 2004. 
[161] S. Catheline et al., "Measuring of viscoelastic properties of homogeneous soft solid using transient elastography: an inverse problem approach.," J. Acoust. Soc. Am., vol. 116, no. 6, pp. 3734-3741, 2004.

[162] H. Xu et al., "Axial-shear strain imaging for differentiating benign and malignant breast masses," Ultrasound Med. Biol., vol. 36, no. 11, pp. 1813-1824, 2010.

[163] E. R. Dougherty and R. a Lotufo, Hands-on Morphological Image Processing. Bellingham: SPIE PRESS, 2003.

[164] D. Ioannou, W. Huda, and A. F. Laine, "Circle recognition through a 2D Hough Transform and radius histogramming," Image Vis. Comput., vol. 17, pp. 15-26, 1999.

[165] G. ter Haar, "Ultrasound focal beam surgery," Ultrasound Med. Biol., vol. 21, no. 9, pp. 1089-1100, 1995.

[166] J. Tavakkoli and N. T. Sanghvi, "Ultrasound-Guided HIFU and Thermal Ablation," in Therapeutic Ultrasound: Mechanisms to Applications, V. Frenkel, Ed. Hauppauge, NY: Nova Science Publishers, 2011, pp. 137-161.

[167] J. Bercoff, M. Pernot, M. Tanter, and M. Fink, "Monitoring thermally-induced lesions with supersonic shear imaging.," Ultrason. Imaging, vol. 26, no. 2, pp. 71-84, 2004.

[168] W. C. Hayes, L. M. Keer, G. Herrmann, and L. F. Mockros, “A mathematical analysis for indentation tests of articular cartilage," J. Biomech., vol. 5, no. 5, pp. 541-551, 1972.

[169] E. L. Carstensen and K. J. Parker, "Physical models of tissue in shear fields," Ultrasound Med. Biol., vol. 40, no. 4, pp. 655-674, 2014.

[170] M. Palmeri, A. Sharma, R. Bouchard, R. Nightingale, and K. Nightingale, "A FiniteElement Method Model of Soft Tissue Response to Impulsive Acoustic Radiation Force," IEEE Trans Ultrason Ferroelectr Freq Control, vol. 13, no. 14-15, pp. 1133-1145, 2005. 
[171] A. P. A. Sarvazyan, O. O. V Rudenko, S. D. Swanson, J. B. Fowlkes, and S. Y. Emelianov, "Shear wave elasticity imaging: a new ultrasonic technology of medical diagnostics," Ultrasound Med. Biol., vol. 24, no. 9, pp. 1419-1435, 1998. 